\title{
Program and Abstracts of the 31st International Symposium on the Autonomic Nervous System
}

\author{
AAS Virtual Meeting \\ November 6-7, 2020 \\ Meeting Program
}

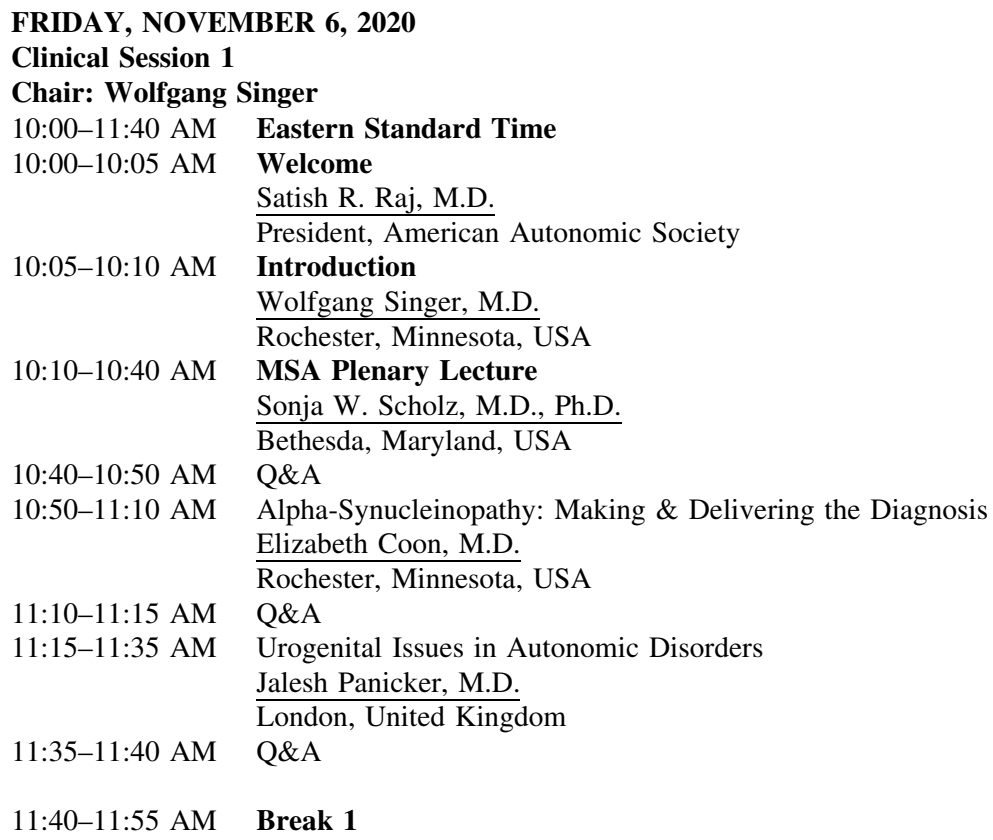

Oral Abstract Session A

Chairs: Victoria Claydon
11:55 AM-1:25 PM
Eastern Standard Time
11:55 AM-12:05 PM

\section{Session Overview}
Victoria Claydon, Ph.D.
Burnaby, BC, Canada
12:05-12:15 PM
Oral Abstract Presentation OA1 (Virtual Poster 27)
Control of heart function by nucleus ambiguus neurons
Tatiana C. Coverdell
12:15-12:20 PM
Charlottesville, Virginia, USA
Q\&A 
12:20-12:30 PM Oral Abstract Presentation OA2 (Virtual Poster 4)

Pathophysiological meaning of alpha-synuclein deposition in sympathetic noradrenergic nerves in skin, submandibular gland, and heart

Risa Isonaka, Ph.D.

12:30-12:35 PM Bethesda, Maryland, USA Q\&A

Oral Abstract Presentation OA3 (Virtual Poster 35)

Muscle metaboreflex control of sympathetic activity is preserved in healthy males following acute intermittent hypercapnic hypoxia

Brooke M. Shafer

Kelowna, BC, Canada

Q\&A

Lightning Abstract Presentation LA1 (Virtual Poster 1)

Sympathetic noradrenergic deficiency in Lewy body diseases is cardioselective

Guillaume Lamotte, M.D.

Bethesda, Maryland, USA

Lightning Abstract Presentation LA2 (Virtual Poster 2)

Cutaneous alpha-synuclein deposition across the synucleinopathies

Christopher H. Gibbons, M.D.

Boston, Massachusetts, USA

1:00-1:05 PM Lightning Abstract Presentation LA3 (Virtual Poster 3)

Alpha-synuclein oligomers and neurofilament light chain in spinal fluid differentiate multiple system atrophy from Lewy body synucleinopathies

Wolfgang Singer, M.D.

Rochester, Minnesota, USA

1:05-1:10 PM Lightning Abstract Presentation LA4 (Virtual Poster 34)

The effect of analgesic fentanyl administration on sympathetic and cardiovascular responses in humans during simulated hemorrhage

Joseph C. Watso, Ph.D.

Dallas, Texas, USA

1:10-1:15 PM Lightning Abstract Presentation LA5 (Virtual Poster 19)

Activation of the rostral ventrolateral medulla results in differential increases in adrenal versus splanchnic sympathetic nerve activity

Soumya S. Kulkarni, M.Sc.

Detroit, Michigan, USA

$1: 15-1: 25$ PM

Open Panel Q\&A

$1: 25-1: 40 \mathrm{PM}$

Break 2

Interactive Clinical Case Presentations: The 3rd Ultimate Autonomic Challenge

Organizer: Juan Guzman

1:40-3:10 PM

$1: 40-1: 45$ PM

1:45-2:00 PM

2:00-2:10 PM

$2: 10-2: 25 \mathrm{PM}$

2:25-2:35 PM

2:35-2:50 PM

2:50-3:00 PM

3:00-3:10 PM
Eastern Standard Time

Introduction

Juan Guzman, M.D.

Hamilton, ON, Canada

Clinical Case 1

Moderator: Howard Snapper

Q\&A

Clinical Case 2

Moderator: Mohammed Numan

Q\&A

Clinical Case 3

Moderator: Steven Vernino

Q\&A

Open Mic and Closing Remarks

SATURDAY, NOVEMBER 7, 2020

Clinical Session 2: Debates

Chair: Steven Vernino

10:00-11:25 AM Eastern Standard Time 
10:00-10:05 AM Welcome

Steven Vernino, M.D., Ph.D.

Dallas, Texas, USA

10:05-10:45 AM IVIG Debate: "IVIG should be used broadly in POTS patients with autoimmune disorders"

IVIG Debate Intro (2 min)

Steven Vernino, M.D. Ph.D.

Dallas, Texas, USA

PRO-IVIG should be used broadly (10 min)

Jill R. Schofield, M.D.

Denver, CO, USA

CON-IVIG should NOT be used broadly (10 min)

Christopher H. Gibbons, M.D.

Boston, Massachusetts, USA

PRO Rebuttal—Jill R. Schofield (5 min)

CON Rebuttal-Christopher H. Gibbons (5 min)

Q\&A (8 min)

10:45-11:25 AM Peds Debate: "We should not require a heart rate increase of 40 bpm to diagnose POTS in kids"

Peds Debate Intro (2 min)

Mohammed Numan, M.D.

Houston, Texas, USA

PRO-We should NOT require the HR increase (10 min)

Blair P. Grubb, M.D.

Toledo, Ohio, USA

CON-We should require the HR increase (10 min)

Philip Fischer M.D., Ph.D.

Rochester, Minnesota, USA

PRO Rebuttal-Blair P. Grubb (5 min)

CON Rebuttal—Philip Fisher (5 min)

Q\&A (8 min)

\section{1:25-11:40 AM Break 3}

Oral Abstract Session B

Chair: Amy Arnold

11:40 AM-1:10 Eastern Standard Time

PM

\section{1:40-11:50 AM Session Overview}

Amy Arnold, Ph.D.

Hershey, Pennsylvania, USA

11:50-12:00 PM Oral Abstract Presentation OB1 (Virtual Poster 70)

Chronic transcutaneous vagal nerve stimulation in hyperadrenergic POTS patients

Dana Shiffer, M.D.

Milan, Italy

12:00-12:05 PM

Q\&A

12:05-12:15 PM

12:15-12:20 PM

Oral Abstract Presentation OB2 (Virtual Poster 56)

Midodrine improves quality of life in patients with vasovagal syncope: results from the Prevention of Syncope Trial IV Lucy Y. Lei, B.Sc.

Calgary, AB, Canada

Oral Abstract Presentation OB3 (Virtual Poster 57)

Transient early orthostatic hypotension-a subtype of orthostatic hypotension

Priyanka Shekhawat, M.D.

Boston, Massachusetts, USA

Q\&A

\section{Lightning Abstract Presentation LB1 (Virtual Poster 36)}

In-vivo recordings from the human vagus nerve using ultrasound-guided microneurography

Vaughan G. Macefield, Ph.D.

Melbourne, Australia

12:40-12:45 PM Lightning Abstract Presentation LB2 (Virtual Poster 37)

Evidence of sympathetic regulation of the choroid circulation by baroreceptors in humans

Seth W. Holwerda, Ph.D.

Iowa City, Iowa, USA 
12:45-12:50 PM Lightning Abstract Presentation LB3 (Virtual Poster 53)

Orthostatic blood pressure and arterial stiffness in persons with spinal cord injury: the effect of the renin-angiotensinaldosterone system

Caitlyn G. Katzelnick

Bronx, New York, USA

12:50-12:55 PM Lightning Abstract Presentation LB4 (Virtual Poster 54)

Frequency of injuries associated with syncope in the prevention of syncope trials

Juliana G. Jorge

Calgary, AB, Canada

12:55-1:00 PM Lightning Abstract Presentation LB5 (Virtual Poster 55)

Characterizing the physiology of an active stand in initial orthostatic hypotension

Nasia Sheikh, B.Sc.

Calgary, AB, Canada

1:00-1:10 PM Open Panel Q\&A

1:10-1:25 PM Break 4

Clinical Session 3

Chair: Satish Raj

1:25-3:00 PM Eastern Standard Time

1:25-1:30 PM Welcome

Satish R. Raj, M.D.

Calgary, AB, Canada

1:30-2:10 PM Syncope Debate: "Head-Up Tilt Tests have no role in the investigation of syncope"

Syncope Debate Intro (2 min)

Satish R. Raj, M.D.

Calgary, AB, Canada

PRO-HUT have NO role in syncope workup (10 min)

Benjamin D. Levine, M.D.

Dallas, Texas, USA

CON-HUT does have a role in syncope workup $(10 \mathrm{~min})$

Robert Sheldon, M.D., Ph.D.

Calgary, AB, Canada

PRO Rebuttal-Benjamin D. Levine (5 min)

CON Rebuttal-Robert Sheldon (5 min)

Q\&A (8 min)

2:10-2:30 PM Headache \& POTS; When to think about Spontaneous CSF Leaks

Melissa Cortez, D.O.

Salt Lake City, Utah, USA

2:30-2:35 PM Q\&A

2:35-3:00 PM AAS Business Meeting and Closing Remarks

Satish R. Raj, M.D.

President, American Autonomic Society

\section{AAS VIRTUAL POSTER SESSION}

FRIDAY, NOVEMBER 6-SATURDAY, NOVEMBER 7, 2020

\section{AUTONOMIC FAILURE: PAF, MSA, PARKINSON'S DISEASE}

Virtual Poster Sympathetic noradrenergic deficiency in Lewy body diseases is cardioselective

\#1 G. Lamotte, C. Holmes, P. Sullivan, D.S. Goldstein

Bethesda, Maryland, USA

Virtual Poster Cutaneous alpha-synuclein deposition across the synucleinopathies

\#2 C.H. Gibbons, N. Wang, S. Rajan, D.S. Kern, J.A. Palma, H. Kaufman, R. Freeman

Boston, Massachusetts, USA

Virtual Poster Alpha-synuclein oligomers and neurofilament light chain in spinal fluid differentiate multiple system atrophy from Lewy \#3 body synucleinopathies

W. Singer, A.M. Schmeichel, M. Shahnawaz, J.D. Schmelzer, B.F. Boeve, D.M. Sletten, T.L. Gehrking, J.A. Gehrking, A.D. Olson, R. Savica, M.D. Suarez, C. Soto, P.A. Low

Rochester, Minnesota, USA

Virtual Poster Pathophysiological meaning of alpha-synuclein deposition in sympathetic noradrenergic nerves in skin, submandibular gland, \#4 and heart

R. Isonaka, P. Sullivan, D.S. Goldstein

Bethesda, Maryland, USA 
Virtual Poster Intervening effects of orthostatic blood pressure change on subcortical atrophy and cognition in de novo and drug-naïve

\#5 Parkinson's disease

S.-W. Yoo, J.-S. Kim

Seoul, Republic of Korea

Virtual Poster Delayed orthostatic hypotension in Parkinson's disease

\#6 $\quad$ S.-W. Yoo, J.-S. Kim

Seoul, Republic of Korea

Virtual Poster Hemodynamic effects and cardiovascular safety of once-daily oral ampreloxetine (TD-9855), a norepinephrine reuptake

\#7 inhibitor, in healthy adults and subjects with neurogenic orthostatic hypotension

S.R. Raj, L. Norcliffe-Kaufmann, W. Wang, B. Haumann, R. Vickery

Calgary, AB, Canada

Virtual Poster Integration of palliative medicine into a multidisciplinary clinic for multiple system atrophy

\#8 E.A. Coon, P. Stevens, C. Chou, M. Suarez, P. Sandroni, J.H. Bower, F. Ali, P.A. Low, W. Singer, L. Rhee

Rochester, Minnesota, USA

Virtual Poster Association between cardiovascular autonomic control and work ability index in patients with pure autonomic failure

\#9 A.R. Zamunér, B. Cairo, A. Porta, D. Shiffer, R. Fornerone, M. Minonzio, A. Herrera-Santelices, R. Furlan, F. Barbic Maule, Chile

Virtual Poster Objective longitudinal evaluation of autonomic function in isolated REM sleep behavior disorder

\#10 L. Baldelli, A. Querzani, L. Sambati, G. Calandra-Buonaura, P. Guaraldi, F. Baschieri, F. Provini, P. Cortelli Bologna, Italy

Virtual Poster Symptoms of sympathetic dysfunction in patients with synucleinopathy referred for evaluation in a Chilean autonomic

\#11 laboratory

J. Idiaquez, J.C. Casar, J.F. Idiaquez, R. Iturriaga

Santiago, Chile

Virtual Poster Decreased sympathetic activity is associated with cognitive decline in Parkinson's disease

\#12 G.R. Popov, M.A. Lebedeva, D.V. Zhuravlev, A.A. Yakovlev, A.B. Guekht

Moscow, Russia

Virtual Poster M-STAR, an ongoing Phase 3 study in participants with multiple system atrophy-baseline characteristics

\#13 J.A. Palma, A. Ellenbogen, R. Freeman, G. Höglinger, G. L'Italien, H. Kaufmann, A. Lang, P. Low, W. Meissner, H. Morris, S. Perlman, W. Poewe, J. Schmahmann, W. Singer, S. Vernino, G. Wenning, V. Wirtz, I.A. Qureshi; for the M-STAR Study Investigators

New York, New York, USA

\title{
AUTONOMIC NEUROPATHIES: DIABETIC, AUTOIMMUNE AND OTHER
}

\author{
Virtual Poster Inflammatory small fiber sensory and autonomic neuropathy with prominent dysgeusia \\ \#14 J. Fernandez, G. Cook \\ Bethesda, Maryland, USA \\ Virtual Poster Sudomotor dysfunction in taxanes-induced peripheral neurotoxicity (CIPN) graded using the Total Neuropathy Score- \\ \#15 Reduced in breast cancer patients \\ S.-Y. Kim \\ Ulsan, Korea \\ Virtual Poster Response to cold stress stimulus in diabetic vasculopathy: a pulse volume study \\ \#16 S. Tripathi, E. Khandelwal \\ Raipur, India \\ Virtual Poster Does subclinical vitamin B12 deficiency alter the normal autonomic modulation during pregnancy? \\ \#17 S. Sharma, S. Sucharita \\ Bangalore, India \\ Virtual Poster Small fiber neuropathy among Gulf War Veterans evaluated at the WRIISC \\ \#18 E.C. Shadiack, O. Osinubi, C. Bhate, A. Gruber-Fox, L. Patrick-DeLuca, P. Cohen, D. Helmer \\ East Orange, New Jersey, USA
}

\section{AUTONOMIC REGULATION: BASIC SCIENCE \& ANIMAL STUDIES}

Virtual Poster Activation of the rostral ventrolateral medulla results in differential increases in adrenal versus splanchnic sympathetic nerve \#19 activity S.S. Kulkarni, N.A. Mischel, P.J. Mueller

Detroit, Michigan, USA

Virtual Poster Cerebral autoregulation associated with falls in older persons with orthostatic hypotension?

\#20 N.I. Saedon, C.H. Goh, J. Frith, W.A.W. Ahmad, K.S. Tan, S.B. Kamaruzzaman, M.P. Tan

Kuala Lumpur, Malaysia

Virtual Poster Norepinephrine transporter saturation at physiological sympathetic firing rates in the murine heart

\#21 L.L. Cao, J.M. Marshall, L. Fabritz, K.L. Brain

Birmingham, United Kingdom

Virtual Poster Direct recording of rat vagal tone

\#22 J.T. Marmerstein, G.A. McCallum, D.M. Durand

Cleveland, Ohio, USA 
Virtual Poster Vagal reactivation after stress testing is associated with vagal deactivation during orthostatic testing in women in the luteal \#23 phase of the menstrual cycle G.M.R. Cunha, E.M.K.K. Soares, C.J.G. da Cruz, L.G.G. Porto, D.S. Martin, M. Morlim, G.E. Molina Brasília, Brazil

Virtual Poster High fat diet-induced adaptations in cardiac vagal motor neuron electrophysiology in the pathogenesis of cardiovascular \#24 disease L. Espinoza, C.R. Boychuk

San Antonio, Texas, USA

Virtual Poster Evidence for signaling plasticity in preganglionic parasympathetic motor neurons in the dorsal motor nucleus of the vagus as a \#25 contributor to the regulation of pancreatic alpha cell regulation S. Fedorchak, M. Lopez, T. Fujikawa, C.R. Boychuk San Antonio, Texas, USA

Virtual Poster Cardiovascular deconditioning induces synaptic and neuronal plasticity in the nucleus tractus solitarii of rats

\#26 L. Lima-Silveira, D. Martinez, E.M. Hasser, D.D. Kline Columbia, Missouri, USA

\section{CARDIAC AUTONOMIC INNERVATION: HUMANS}

\begin{tabular}{|c|c|c|}
\hline $\begin{array}{l}\text { Virtual } \\
\# 27\end{array}$ & Poster & $\begin{array}{l}\text { Control of heart function by nucleus ambiguus neurons } \\
\text { T.C. Coverdell, R.-J. Abraham-Fan, N.M. Schiavone, M. Crook, S.B. Abbott, J.N. Campbell } \\
\text { Charlottesville, Virginia, USA }\end{array}$ \\
\hline $\begin{array}{l}\text { Virtual } \\
\# 28\end{array}$ & Poster & $\begin{array}{l}\text { Deep breathing impacts blood pressure to a greater degree in individuals with cervical spinal cord injury } \\
\text { controls } \\
\text { M.T Maher, J.P. Weir, W.A. Bauman, J.M. Wecht } \\
\text { Bronx, New York, USA }\end{array}$ \\
\hline $\begin{array}{l}\text { Virtual } \\
\# 29\end{array}$ & Poster & $\begin{array}{l}\text { Impact of angiotensin receptor neprilysin inhibitors on blood pressure variability in heart failure } \\
\text { S. Nathaniel, S. McGinty, D.G. Edwards, W.B. Farquhar, M.A.H. Witman, V. Hosmane, M.M. Wenner } \\
\text { Newark, Delaware, USA }\end{array}$ \\
\hline $\begin{array}{l}\text { Virtual } \\
\# 30\end{array}$ & Poster & $\begin{array}{l}\text { Assessment of endothelial dysfunction in sickle cell disease-an insight into vaso-occlusive crisis } \\
\text { E. Khandelwal, M. Kumar, S. Tripathi } \\
\text { Raipur, India }\end{array}$ \\
\hline $\begin{array}{l}\text { Virtual } \\
\# 31\end{array}$ & Poster & $\begin{array}{l}\text { Respiratory neuroplasticity to acute intermittent hypercapnic hypoxia in humans } \\
\text { C.V. Brown, T.D. Vermeulen, B.M. Shafer, T.J.R. Stuckless, G.E. Foster } \\
\text { Kelowna, BC, Canada }\end{array}$ \\
\hline $\begin{array}{l}\text { Virtual } \\
\text { \#32 }\end{array}$ & Poster & $\begin{array}{l}\text { Orthostatic tachycardia and functional cardiac response following strict head-down-tilt bedrest } \\
\text { F. Hoffmann, J. Rabineau, D. Mehrkens, B.W. Johannes, E. Caiani, P.-F. Migeotte, J. Jordan, J. Tank } \\
\text { Cologne, Germany }\end{array}$ \\
\hline $\begin{array}{l}\text { Virtual } \\
\# 33\end{array}$ & Poster & $\begin{array}{l}\text { Heart rate variability in healthcare workers with and without preschool children } \\
\text { B. De Maria, V. De Grazia, G. Cassetti, L. Clementi, F. Perego, A. Porta, L.A. Dalla Vecchia } \\
\text { Milan, Italy }\end{array}$ \\
\hline
\end{tabular}

\section{CARDIOVASCULAR AUTONOMIC REFLEXES IN HUMANS, INCLUDING MICRONEUROGRAPHY}

Virtual Poster The effect of analgesic fentanyl administration on sympathetic and cardiovascular responses in humans during simulated \#34 hemorrhage J.C. Watso, M. Huang, L.N. Belval, F.A. Cimino III, J.M. Hendrix, C. Hinojosa-Laborde, C.G. Crandall Dallas, Texas, USA

Virtual Poster Muscle metaboreflex control of sympathetic activity is preserved in healthy males following acute intermittent hypercapnic \#35 hypoxia B.M. Shafer, T.D. Vermeulen, A.V. Incognito, M. Nardone, A.L. Teixeira, J. Benbaruj, P.J. Millar, G.E. Foster Kelowna, BC, Canada

Virtual Poster In-vivo recordings from the human vagus nerve using ultrasound-guided microneurography

\#36

Virtual Poster

\#37

Virtual Poster \#38

Virtual Poster \#39

Virtual Poster \#40
M.M. Ottaviani, L. Wright, T. Dawood, V.G. Macefield

Melbourne, Australia

Evidence of sympathetic regulation of the choroid circulation by baroreceptors in humans

S.W. Holwerda, R.H. Kardon, R. Hashimoto, J.K. Nellis, J.M. Full, G.L. Pierce Iowa City, Iowa, USA

Muscle sympathetic single-unit response patterns during graded muscle metaboreflex activation in young healthy adults A.V. Incognito, M. Nardone, A.L. Teixeira, J.B. Lee, M.M. Kathia, P.J. Millar Guelph, ON, Canada

Six months of exercise-based cardiac rehabilitation enhances sympathetic neural recruitment strategies during maximal endinspiratory apnea

A.W. D’Souza, M.B. Badrov, S. Lalande, N. Suskin, J.K. Shoemaker

London, ON, Canada

Effect of sensory blockade and rate of sensory stimulation on local heating induced axon reflex response in facial skin K. Metzler-Wilson, T.E. Wilson, S.M. Ausmus, A. Sventeckis Indianapolis, Indiana, USA 


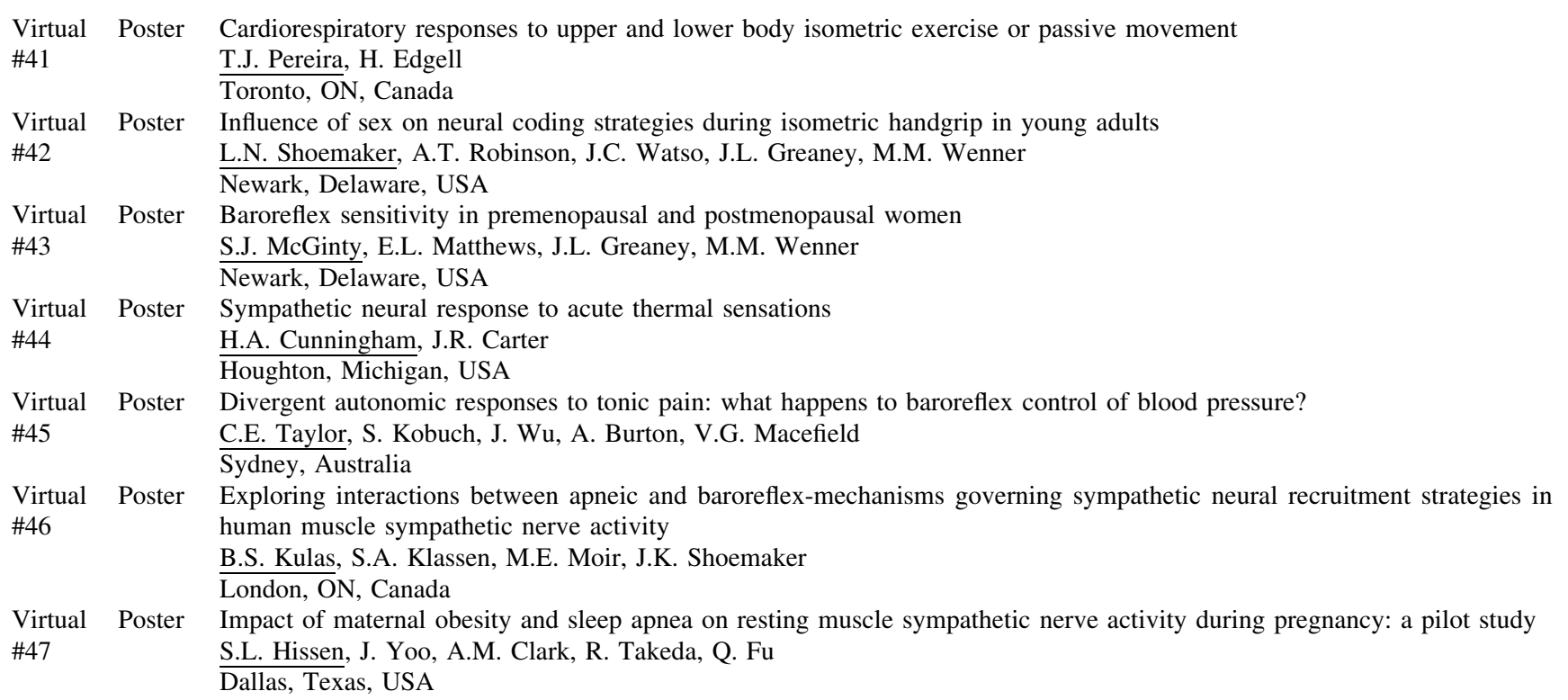

\section{CEREBRAL BLOOD FLOW REGULATION}

Virtual Poster \#48 Elevated cerebral blood flow in individuals with pure autonomic failure

K.R. Hay, P. Trujillo, O.C. Roman, S.Y. Paranjape, E.M. Garland, C.A. Shibao, I. Biaggioni, M.J. Donahue, D.O. Claassen Nashville, Tennessee, USA

Virtual Poster \#49 Cerebrovascular compliance is regulated by sympathetic and cholinergic inputs M.E. Moir, J.W. Hamner, C.O. Tan, J.K. Shoemaker London, ON, Canada

\section{GASTROINTESTINAL \& UROGENITAL SYSTEMS, IBS, CYSTITIS}

Virtual Poster Impact of patisiran, an RNAi therapeutic, on diarrhea symptoms in patients with hereditary transthyretin-mediated \#50 amyloidosis

L. Obici, A. González-Duarte, M. Waddington-Cruz, Q. Dinh, H. Lin, M. Merkel, Y. Wang, M. Ueda

Cambridge, Massachusetts, USA

Virtual Poster Quantifiable gastrointestinal dysmotility in idiopathic autonomic neuropathy

\#51 J. Langford, L. Millsap, L.A. Pace, J. Hemp, M.M. Cortez

Salt Lake City, Utah, USA

Virtual Poster Gastrointestinal dysmotility is common in autonomic nervous system disorders and has important implications for clinical \#52 management

L.A. Pace, J. Hemp, J. Langford, L. Millsap, D. Ball, M.M. Cortez

Salt Lake City, Utah, USA

\section{ORTHOSTATIC HYPOTENSION \& SYNCOPE}

Virtual Poster Orthostatic blood pressure and arterial stiffness in persons with spinal cord injury: the effect of the renin-angiotensin\#53 aldosterone system C.G. Katzelnick, J.P. Weir, G.P. Zipp, M.F. LaFountaine, J.M. Wecht

Bronx, New York, USA

Virtual Poster Frequency of injuries associated with syncope in the prevention of syncope trials

\#54 J.G. Jorge, P. Pournazari, S.R. Raj, C. Maxey, R.S. Sheldon

Calgary, AB, Canada

Virtual Poster Characterizing the physiology of an active stand in initial orthostatic hypotension

\#55 N. Sheikh, S. Ranada, M. Lloyd, D. McCarthy, R.S. Sheldon, A. Phillips, D.V. Exner, M. Runte, S.R. Raj Calgary, AB, Canada

Virtual Poster Midodrine improves quality of life in patients with vasovagal syncope: results from the Prevention of Syncope Trial IV

\#56 L.Y. Lei, R.S. Sheldon, S. Safdar, D. Ritchie, C. Maxey, S.R. Raj Calgary, AB, Canada

Virtual Poster Transient early orthostatic hypotension - a subtype of orthostatic hypotension

\#57 P. Shekhawat, K. Senechal, V. Galvis, I. Bonyhay, C. Gibbons, R. Freeman

Boston, Massachusetts, USA 
Virtual Poster Effect of the initial maintenance dose of droxidopa on treatment persistence in patients with neurogenic orthostatic \#58 hypotension

F. Amjad, L.A. Hewitt, S. Kymes, B. Polenchar, A. Favit

Deerfield, Illinois, USA

Virtual Poster A comparison of the Active Standing Test (AST) and Head-Up Tilt Test (HUTT) in children and young people

\#59 K. Vakili, D. Moore, G. Parker, W.P. Whitehouse

Nottingham, United Kingdom

Virtual Poster Likelihood of injury due to vasovagal syncope. A systematic review and meta-analysis

\#60 J.G. Jorge, S.R. Raj, R.S. Sheldon

Calgary, AB, Canada

Virtual Poster Impact of orthostatic hypotension in inpatient rehabilitation

\#61 P. Sharma, L.E. Okamoto, L. Massey, I. Biaggioni, J. Johns

Nashville, Tennessee, USA

Virtual Poster Impact of patisiran, an RNAi therapeutic, on orthostatic intolerance in patients with hereditary transthyretin-mediated

$\# 62$ amyloidosis

D.P. Judge, A. González-Duarte, A. Dispenzieri, H. Lin, M. Merkel, Y. Wang, M. Polydefkis

Mexico City, Mexico

Virtual Poster Activating lower body muscles prior to standing mitigates symptoms of initial orthostatic hypotension

\#63 N. Sheikh, S. Ranada, M. Lloyd, D. McCarthy, R.S. Sheldon, A. Phillips, D.V. Exner, M. Runte, S.R. Raj

$\overline{\text { Calgary, AB, Canada }}$

Virtual Poster Autonomic function testing in patients with end-stage heart failure following continuous-flow left ventricular assist device \#64 implantation

M.G. Lloyd, J. Jorge, J. Angihan, D. Chew, L.Y. Lei, K. Kogut, N. Sharma, J. Howlett, N. Fine, S.R. Raj

Calgary, $\mathrm{AB}$, Canada

Virtual Poster Assessing the relationship between orthostatic hypotension and cognitive impairment

\#65 J. Baker, Malcolm Sherwood, Jennifer Fogarty, Michael Borrie, Jaspreet Bhangu

London, ON, Canada

\section{PEDIATRIC AUTONOMIC DISORDERS}

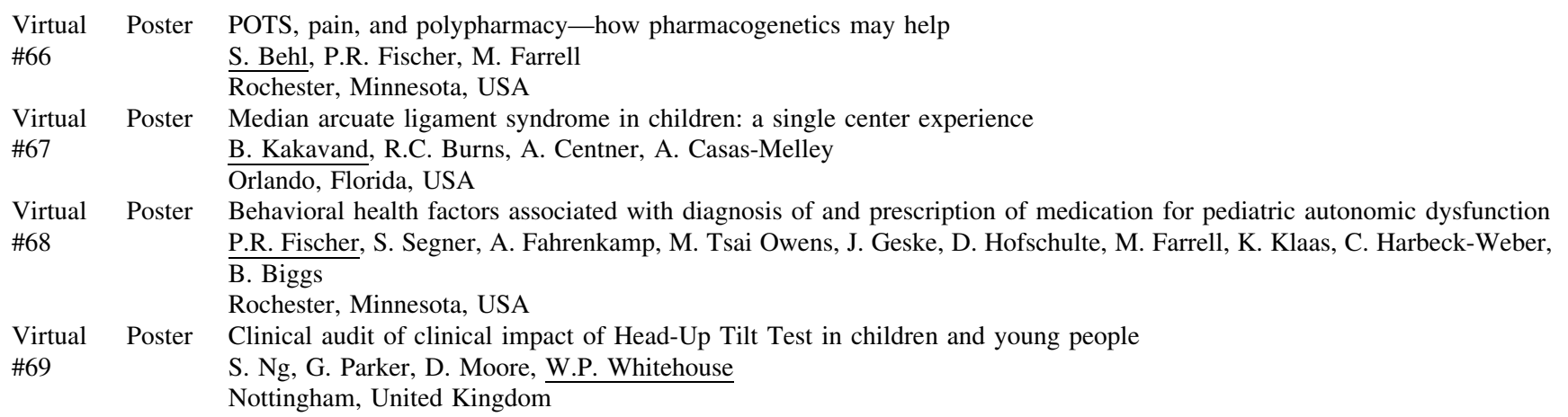

\section{POSTURAL TACHYCARDIA SYNDROME (POTS)}

Virtual Poster Chronic transcutaneous vagal nerve stimulation in hyperadrenergic POTS patients

\#70 D. Shiffer, R. Furlan, F. Barbic, R. Zamuner, M. Minonzio, F. Dipaola, B. Cairo, A. Porta, S. Rigo, V. Mundula, E. Tobaldini, L. Furlan, N. Montano, I. Biaggioni, A. Diedrich

Milan, Italy

Virtual Poster Perception of cognitive impairment in individuals with POTS

\#71 E.M. Rich, C. Hollingsworth, V. Boyette, A. Vas

Dallas, Texas, USA

Virtual Poster Screening for novel autoantibodies in POTS

\#72 S. Vernino, M. Bryarly, R. Schubert, M.R. Wilson

Dallas, Texas, USA

Virtual Poster Work ability assessment in active workers with postural tachycardia syndrome

\#73 S. Cavalieri, M. Minonzio, F. Dipaola, D. Shiffer, B. Cairo, R.A. Zamuner, I. Capitanelli, N. Magnavita, R. Furlan, F. Barbic Rome, Italy

Virtual Poster Relationship between work ability and cardiac autonomic profile in postural tachycardia syndrome

\#74 F. Barbic, M. Minonzio, D. Shiffer, F. Dipaola, B. Cairo, R.A. Zamuner, S. Cavalieri, I. Capitanelli, N. Magnavita, R. Furlan Milan, Italy

Virtual Poster Co-occurrence of POTS and myasthenia gravis

\#75 R. Shaik, D. Sinn, S. Jaradeh, S. Muppidi

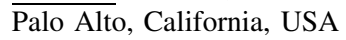


Virtual Poster Immunogenetic risk markers in postural tachycardia syndrome

\#76 Z. Orban, A. Miller, K. Bourne, J. Nitis, W. Hu, R.A. Reinsel, L.E. Stiles, A. Fedorowski, J. Axelsson Chicago, Illinois, USA

Virtual Poster Menstrual irregularities in pediatric patients with postural tachycardia syndrome and chronic orthostatic intolerance

\#77 L. Riordan, K. Leopold, A. Chattha, E. Bolen, W. Bunn, S. Hasan, K. Klaas

Rochester, Minnesota, USA

Virtual Poster Subcutaneous immunoglobulin in patients with postural tachycardia syndrome

\#78 K. Kesterson, S. Blitshteyn

Buffalo, New York, USA

Virtual Poster The utilization of salt and electrolytes in the treatment of postural tachycardia syndrome

\#79 J.E. Hall, K. Bourne, L.E. Stiles, R.S. Sheldon, C.A. Shibao, L.E. Okamoto, E.M. Garland, A. Peltier, A. Diedrich, I. Biaggioni, A. Gamboa, S.R. Raj

Calgary, AB, Canada

Virtual Poster A comparison of health-related quality of life in autonomic disorders: postural tachycardia syndrome vs. vasovagal syncope

\#80 J.E. Hall, J. Ng, K. Bourne, R.S. Sheldon, M. Bryarly, S. Vernino, A. Gamboa, S.R. Raj

Calgary, AB, Canada

Virtual Poster Retrospective review of subjective responses to carbidopa in autonomic disorders

\#81 C.J. Park, E.P. Golden, S. Vernino

Dallas, Texas, USA

Virtual Poster Time course of autonomic symptoms in postural tachycardia syndrome (POTS) patients: two-year follow-up results

\#82 F. Dipaola, C. Barberi, E. Castelnuovo, M. Minonzio, D. Shiffer, B. Cairo, A. Porta, F. Barbic, R. Furlan

Milan, Italy

Virtual Poster POTS patients with excessive orthostatic tachycardia experience more benefits from body compression than patients with less \#83 orthostatic tachycardia

K.M. Bourne, J. Hall, R.S. Sheldon, D.V. Exner, J. Tyberg, S.R. Raj

Calgary, AB, Canada

Virtual Poster Phenylephrine alters phase synchronization between cerebral blood flow and blood pressure during orthostasis: effect on \#84 N-back performance in chronic fatigue syndrome/postural tachycardia syndrome

M.S. Medow, C. Terilli, J.M. Stewart

Valhalla, New York, USA

Virtual Poster Associated comorbid conditions contribute to gastrointestinal symptoms in postural tachycardia syndrome

\#85 L. Millsap, J. Langford, J. Hemp, M.M. Cortez, L.A. Pace

Salt Lake City, Utah, USA

\section{OTHER AUTONOMIC DISORDERS}

Virtual Poster When sinus tachycardia becomes too much: negative effects of excessive upright tachycardia on cardiac output in vasovagal \#86 syncope, postural tachycardia syndrome, and inappropriate sinus tachycardia

J.M. Stewart, M.S. Medow, P. Visintainer, R. Sutton

Valhalla, New York, USA

Virtual Poster The "extended" ANS, stress, and multi-system, multi-disciplinary disorders of regulation

\#87 D.S. Goldstein

Bethesda, Maryland, USA

Virtual Poster Prevalence and patterns of abnormal QSWEAT volumes: a retrospective review

\#88 T. Prieto, D.I. Sinn, S. Muppidi, M.G. Miglis, R. Shaik, S. Jaradeh

Stanford, California, USA

Virtual Poster Chronic use of baclofen and gabapentin in non-ambulatory, motor-incomplete persons with spinal cord injury modulates the \#89 change in systolic blood pressure to the seated upright posture

M.F. LaFountaine, J.P. Weir, C.G. Katzelnick, M.T. Maher, J.M. Wecht

Bronx, New York, USA

Virtual Poster A cross sectional survey of autonomic nervous system dysfunction in patients with mitochondrial disease

\#90 E.C. Shadiack III, J.R. Boris, L. MacMullen, E.M. McCormick, I. George-Sankoh, M.J. Falk

Philadelphia, Pennsylvania, USA

\section{Accreditation and Credit Designation Statements}

This activity has been planned and implemented in accordance with the Essential Areas and Policies of the Accreditation Council for Continuing Medical Education through the joint providership of The University of Texas Southwestern Medical Center and the American Autonomic Society. The University of Texas Southwestern Medical Center is accredited by the ACCME to provide continuing medical education for physicians.

The University of Texas Southwestern Medical Center designates this live activity for a maximum of 10.0 AMA PRA Category 1 Credit( $(s)^{T M}$. Physicians should only claim credit commensurate with the extent of their participation in the activity. 
THE AMERICAN AUTONOMIC SOCIETY WOULD LIKE TO THANK THE FOLLOWING FOR THEIR SUPPORT:

GOLD SPONSOR

Theravance Biopharma $\lambda$.

Medicines That Make a Difference

SILVER SPONSORS
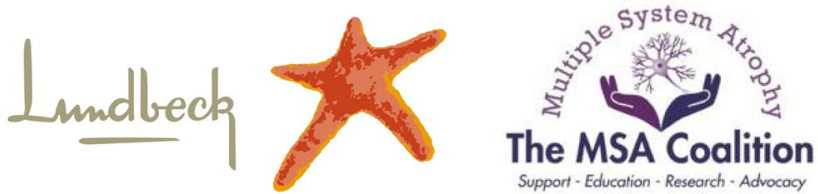

BRONZE SPONSOR

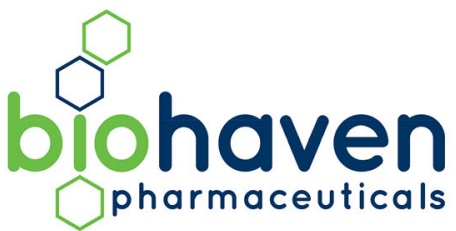




\section{AAS 2020 VIRTUAL POSTERS}

\section{Virtual Poster \#1}

\section{Sympathetic noradrenergic deficiency in Lewy body diseases is cardioselective}

\section{G. Lamotte, C. Holmes, P. Sullivan, D.S. Goldstein}

Autonomic Medicine Section, Clinical Neurosciences Program, Division of Intramural Research, National Institute of Neurological Disorders and Stroke, National Institutes of Health, Bethesda, MD, USA

Objective: Lewy body forms of autonomic synucleinopathy include Parkinson's disease (PD), dementia with Lewy bodies, and pure autonomic failure (PAF). These conditions entail profound myocardial norepinephrine (NE) deficiency, whereas the non-Lewy body synucleinopathy multiple system atrophy (MSA) in most cases does not. Whether the sympathetic noradrenergic lesion in Lewy body synucleinopathies is cardioselective has been unclear. We therefore reviewed 18F-dopamine (18F-DA) positron emission tomographic (PET) images and post-mortem neurochemical data from patients with synucleinopathies and control subjects.

Methods: We analyzed 18F-DA PET scan images from clinical research protocols at the National Institutes of Health Clinical Center between 1990 and 2020 and quantified 18F-DA-derived radioactivity in the heart, liver, spleen, pancreas, stomach, kidneys, thyroid, and submandibular glands. Post-mortem tissue samples from the heart, liver, spleen, pancreas, kidneys, thyroid, submandibular glands and sympathetic ganglia were assayed for NE by liquid chromatography with electrochemical detection. Neuroimaging data were available for 145 patients with Lewy body synucleinopathies (112 PD, 33 PAF), 85 MSA, and 74 controls. Post-mortem tissue NE data were available for $38 \mathrm{PD}, 2 \mathrm{PAF}, 5 \mathrm{MSA}$, and 35 controls.

Results: 18F-DA-derived radioactivity in the interventricular cardiac septum was decreased in the Lewy body synucleinopathy group compared to the control and MSA groups $(\mathrm{p}<0.0001$ by factorial analysis of variance). 18F-DA-derived radioactivity was not decreased in the liver, spleen, pancreas, stomach, kidneys, or submandibular glands in the Lewy body synucleinopathy group. The Lewy body synucleinopathy group had markedly decreased myocardial NE compared to the controls but no decrease in tissue NE contents in any of the other organs.

Interpretation: In Lewy body synucleinopathies peripheral noradrenergic deficiency is cardioselective. Understanding bases for this phenomenon may help elucidate the pathogenesis of these disorders. Funding: This research was supported by the Division of Intramural Research of the National Institute of Neurological Disorders and Stroke (NINDS), NIH.

\section{Virtual Poster \#2}

\section{Cutaneous alpha-synuclein deposition across the synucleinopathies}

C.H. Gibbons, N. Wang, S. Rajan, D.S. Kern, J.A. Palma, H. Kaufman, R. Freeman

Department of Neurology, Beth Israel Deaconess Medical Center, Harvard Medical School, Boston, MA, USA

Background: We have previously reported that $\alpha$-synuclein can be detected within cutaneous nerve fibers of patients with Parkinson's disease (PD) and multiple system atrophy (MSA). We now report the deposition of phosphorylated alpha-synuclein in all forms of synucleinopathy including PD, MSA, dementia with Lewy bodies (DLB) and pure autonomic failure (PAF).

Objective: To define the cutaneous deposition of phosphorylated alpha-synuclein in synucleinopathies.

Methods: 88 patients with synucleinopathy, 75 healthy control and 55 control subjects with concomitant disease (diabetic neuropathy, stroke, Alzheimer's disease) had detailed neurological examinations and autonomic function testing. Skin biopsies were taken from the posterior cervical, proximal and distal thigh, and were stained for PGP9.5 and phosphorylated alpha-synuclein.

Results: Phosphorylated alpha-synuclein was detected in 41/42 patients with PD, 17/17 with MSA, 14/14 with DLB, 14/15 with PAF, 0/75 healthy controls and 0/55 disease controls. $98 \%$ of synucleinopathy patients were positive in 1 or more biopsies, and none of the healthy or disease controls were positive. Moderate correlations were noted between disease duration, disease severity (as measured by validated scales) and phosphorylated synuclein deposition (correlations $0.42-0.61, \mathrm{P}<0.01$ ).

Discussion: We report the largest study of cutaneous phosphorylated alpha synuclein detection in patients with all forms of synucleinopathy. Results were similar across the various clinical synucleinopathy phenotypes and demonstrated a clinical correlation with quantified examination scores. These results carry significant implications for disease diagnosis, prognosis and therapeutic interventions that alter the natural history of the disease.

Funding: Study supported by the MSA Foundation (RF) and NIH U54 NS065736.

\section{Virtual Poster \#3}

Alpha-synuclein oligomers and neurofilament light chain in spinal fluid differentiate multiple system atrophy from Lewy body synucleinopathies

W. Singer ${ }^{1}$, A.M. Schmeichel ${ }^{1}$, M. Shahnawaz ${ }^{2}$, J.D. Schmelzer ${ }^{1}$, B.F. Boeve ${ }^{1}$, D.M. Sletten ${ }^{1}$, T.L. Gehrking ${ }^{1}$, J.A. Gehrking ${ }^{1}$, A.D. Olson ${ }^{1}$, R. Savica ${ }^{1}$, M.D. Suarez ${ }^{1}$, C. Soto ${ }^{2}$, P.A. Low ${ }^{1}$ ${ }^{1}$ Department of Neurology, Mayo Clinic, Rochester, MN, USA; ${ }^{2}$ Mitchell Center for Alzheimer's Disease and Related Brain Disorders, Department of Neurology, University of Texas McGovern Medical School at Houston, Houston, TX, USA

Objective: To explore the role of alpha-synuclein ( $\alpha$ Syn) oligomers and neurofilament light chain (NFL) in cerebrospinal fluid (CSF) as markers of early multiple system atrophy (MSA) and to contrast findings to Lewy body synucleinopathies.

Methods: In a discovery cohort of well-characterized early MSA patients $(n=24)$ and matched healthy controls $(C O N, n=14)$ we utilized ELISA to measure NFL and protein misfolding cyclic amplification (PMCA) to detect $\alpha$ Syn oligomers in CSF. We confirmed findings in a separate prospectively enrolled cohort of patients with early MSA ( $\mathrm{n}=38)$, Parkinson's disease (PD, $\mathrm{n}=16)$, dementia with Lewy bodies (DLB, $n=13)$, and CON subjects $(n=15)$.

Results: In the discovery cohort, NFL was markedly elevated in MSA patients with perfect separation from CON. ASyn-PMCA was nonreactive in all $\mathrm{CON}$, while all MSA samples were positive. In the confirmatory cohort, NFL again perfectly separated MSA from CON, and was significantly lower in PD and DLB compared to MSA. PMCA was again non-reactive in all CON, and positive in all but 2 MSA cases. All PD and all but 2 DLB samples were also positive for $\alpha$ Syn aggregates but with markedly different reaction kinetics than MSA: aggregation occurred later but maximum fluorescence was higher, allowing for perfect separation of reactive samples between MSA and Lewy body synucleinopathies. 
Interpretation: NFL and aSyn oligomers in CSF faithfully differentiate early MSA not only from CON but also from Lewy body synucleinopathies. The findings support the role of these markers as diagnostic biomarkers, and have important implications for understanding pathophysiologic mechanisms underlying the synucleinopathies.

Funding: Supported by NIH (R01NS092625, K23NS075141, UL1TR000135, P50AG016574, U01NS100620, R01AG055053, R01AG061069), FDA (R01FD004789), grants from the Michael J. Fox Foundation for Parkinson's disease, Cure MSA Foundation, Sturm Foundation, the Mayo Clinic Dorothy and Harry T. Mangurian Jr. Lewy Body Dementia Program, the Little Family Foundation, and Mayo funds.

\section{Virtual Poster \#4}

Pathophysiological meaning of alpha-synuclein deposition in sympathetic noradrenergic nerves in skin, submandibular gland, and heart

\section{R. Isonaka, P. Sullivan, D.S. Goldstein}

Autonomic Medicine Section, Clinical Neurosciences Program, Division of Intramural Research, National Institute of Neurological Disorders and Stroke, National Institutes of Health, Bethesda, MD, USA

Objective: Autonomic synucleinopathies such as Parkinson's disease (PD) with orthostatic hypotension and pure autonomic failure have in common deposition of the protein alpha-synuclein (AS) in sympathetically innervated tissues and generalized noradrenergic deficiency; however, whether intra-neuronal AS is actually pathogenic in sympathetic neurons has not been tested in any clinical form of synucleinopathy. The purpose of this post-mortem study was to address this issue directly by measuring AS and norepinephrine (NE) in tissue samples from readily biopsiable submandibular gland (SMG) and scalp skin and from myocardium in patients with autopsy-proven PD.

Methods: Immunoreactive AS and tyrosine hydroxylase ( $\mathrm{TH}$, a marker of noradrenergic innervation) were visualized by immunofluorescence confocal microscopy and quantified in coded post-mortem specimens of SMG, scalp, and apical myocardium from 13 PD patients and 14 age-matched control subjects. NE in the same specimens was assayed by liquid chromatography with electrochemical detection.

Results: PD patients had increased AS in SMG (37 times control, $\mathrm{p}<0.0001$ ), all of 3 sympathetic noradrenergically innervated skin constituents-arrector pili muscles, sweat glands, and blood vessels (5.7, 3.8, and 4.0 times control, $\mathrm{p}<0.0001 \mathrm{each})$, and myocardium (15 times control, $\mathrm{p}=0.00018$ ). Across all subjects AS signal intensities in SMG and in the 3 skin constituents correlated positively with myocardial AS and negatively with myocardial TH and NE. Although the PD group had higher mean AS-TH colocalization indices in SMG $(p<0.0001)$ and scalp $(p<0.0001)$ than did the control group, the groups did not differ in $\mathrm{TH}$ or NE contents in either tissue.

Conclusions: Increased immunoreactive AS levels and elevated ASTH colocalization indices in potentially biopsiable SMG and skin tissues are associated with increased myocardial AS deposition, loss of myocardial noradrenergic nerves, and depletion of myocardial NE in PD. Measuring intra-neuronal AS and calculating AS-TH colocalization indices in SMG or skin may provide pathophysiologically relevant biomarkers of cardiac sympathetic neurodegeneration in autonomic synucleinopathies.

Funding: Division of Intramural Research, NINDS, NIH.

\section{Virtual Poster \#5}

Intervening effects of orthostatic blood pressure change on subcortical atrophy and cognition in de novo and drug-naïve Parkinson's disease

\section{S.-W. Yoo, J.-S. Kim}

Department of Neurology, College of Medicine, The Catholic University of Korea, Seoul, Republic of Korea

Background and Purpose: Cognitive impairment and cardiovascular dysautonomia are two major non-motor features of Parkinson's disease (PD). They have been investigated separately and extensively, but their interactive outcomes have rarely been studied. The purpose of this study was to examine the association between central atrophy and cognition and to assess the influence of cardiovascular lability on this association in early PD patients.

Methods: Out of 151 early PD patients, 47 subjects were ultimately enrolled according to our selection criteria. Their cognitive status was examined by comprehensive neuropsychological tests assessing five domains of cognition (attention/working memory, executive function, language, memory, and visuospatial function). Supine and orthostatic blood pressures were recorded during head-up tilt tests, and orthostatic mean arterial pressure change was calculated. Every patient underwent brain magnetic resonance imaging, and intercaudate nucleus ratio was obtained as a central atrophy surrogate marker. The associations and interactions between central atrophy, cognition, and blood pressure variability were analyzed.

Results: Among 47 subjects, 20 (42.6\%) had orthostatic hypotension. Attention/working memory, executive function, and delayed recall were inversely associated with central atrophy $(r=-0.332$, $\mathrm{p}=0.028 ; r=-0.314, \mathrm{p}=0.038 ; r=-0.399, \mathrm{p}=0.024$; respectively). In a multiple regression model, only attention/working memory was independently associated with central atrophy when modulated by orthostatic mean arterial pressure change $(\mathrm{p}<0.05)$. Blood pressure fluctuation worsened this inverse relationship.

Conclusion: This study revealed that cardiovascular dysautonomia interacted with the inverse association between cerebral atrophy and cognition, and it reinforced its relationship. Interaction between these two non-motor features should be kept in mind in clinical practice, particularly in PD patients with co-morbid vascular risk factors.

\section{Virtual Poster \#6}

\section{Delayed orthostatic hypotension in Parkinson's disease}

\section{S.-W. Yoo, J.-S. Kim}

Department of Neurology, College of Medicine, The Catholic University of Korea, Seoul St. Mary's Hospital, Seoul, Republic of Korea

Objective: Orthostatic hypotension $(\mathrm{OH})$ is relatively common in early drug-naïve Parkinson's disease (PD). It is divided into delayed $\mathrm{OH}$ and classical $\mathrm{OH}$. Classical $\mathrm{OH}$ in $\mathrm{PD}$ has been investigated widely, however, the clinical implications of delayed $\mathrm{OH}$ in PD have seldom been studied. The purpose of this study is to characterize delayed $\mathrm{OH}$ in PD.

Methods: A total of 285 patients with early drug-naïve PD were enrolled and divided into three groups according to orthostatic change: no $\mathrm{OH}$, delayed $\mathrm{OH}$, and classical $\mathrm{OH}$. The disease severity was assessed by the Unified Parkinson's Disease Rating Scale and modified Hoehn and Yahr stage. Global cognitive status was evaluated by the Mini-Mental Status Examination and Clinical Dementia Rating. Of 285 patients with PD, 177 were assessed by the Non-Motor 
Symptoms Scale, Parkinson's Disease Quality of Life-39, Orthostatic Hypotension Questionnaire, Montgomery-Asberg Depression Rating Scale, and Rapid-eye-movement Sleep Behavior Disorder Screening Questionnaire. The cortical thickness of 82 patients was analyzed with brain magnetic resonance imaging. Between-group differences and linear tendency in the order of no $\mathrm{OH}$, delayed $\mathrm{OH}$, and classical $\mathrm{OH}$ were investigated. The disease status of 77 patients was reevaluated. Initial and follow-up evaluations were explored to discern any temporal effects of orthostasis on disease severity.

Results: Sixty-four (22.5\%) patients were defined as having delayed $\mathrm{OH}$ and $117(41.1 \%)$ had classical $\mathrm{OH}$. Between-group comparisons revealed that classical $\mathrm{OH}$ had the worst outcomes in motor, nonmotor, cognitive, and cortical thickness, compared to the other groups. No $\mathrm{OH}$ and delayed $\mathrm{OH}$ did not differ significantly. Linear trend analysis across the pre-defined ordinal scale revealed that clinical parameters worsened along with the orthostatic challenge. Clinical scales worsened and the linear gradient was maintained during the follow-up period.

Conclusion: Delayed $\mathrm{OH}$ is a mild form of classical OH in PD. PD with delayed $\mathrm{OH}$ has milder disease severity and progression.

\section{Virtual Poster \#7}

Hemodynamic effects and cardiovascular safety of once-daily oral ampreloxetine (TD-9855), a norepinephrine reuptake inhibitor, in healthy adults and subjects with neurogenic orthostatic hypotension

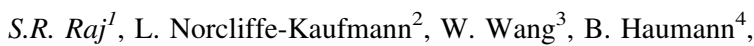
R. Vickery ${ }^{5}$

${ }^{1}$ Cumming School of Medicine, University of Calgary, Calgary, Alberta, Canada; ${ }^{2}$ New York University School of Medicine, Department of Neurology, New York, NY, USA; ${ }^{3}$ Theravance Biopharma US, Inc., South San Francisco, CA, USA; ${ }^{4}$ Theravance Biopharma UK Limited, London, United Kingdom; ${ }^{5}$ Theravance Biopharma Ireland Limited, Dublin, Ireland

Background: In neurogenic orthostatic hypotension ( $\mathrm{nOH})$, inadequate release of norepinephrine (NE) at the neurovascular junction causes blood pressure (BP) to fall on standing and debilitating symptoms of cerebral hypoperfusion. Ampreloxetine is a novel, longacting NE reuptake inhibitor that potentiates the effects of endogenous NE and may increase standing BP and improve symptoms of $\mathrm{nOH}$ in affected patients. Hemodynamic responses and cardiovascular safety of oral ampreloxetine were studied in healthy adults and subjects with $\mathrm{nOH}$.

Methods: Supine and 3-min standing heart rate (HR) and BP, adverse events (AEs), and electrocardiographic parameters were evaluated in a 14-day multiple-dose study in healthy adults $(\mathrm{n}=30$, ampreloxetine $4-40 \mathrm{mg} ; \mathrm{n}=10$ placebo; mean age: $42 \pm 13$ years) and a 20 -week study of open-label ampreloxetine at a median dose of $10 \mathrm{mg}$ in subjects with $\mathrm{nOH}(\mathrm{n}=21$; mean age: $64 \pm 8$ years).

Results: In healthy adults at Day 14 of treatment, ampreloxetine showed a dose-dependent increase in supine HR and systolic BP (SBP). Standing HR and SBP were similar for all treatment groups. Mean change in standing HR did not exceed 25 beats per minute in either healthy adults or subjects with $\mathrm{nOH}$. In healthy adults, there was little change in standing SBP at ampreloxetine $10 \mathrm{mg}$. In subjects with $\mathrm{nOH}$, at a median dose of $10 \mathrm{mg}$, fall in standing SBP improved from baseline by approximately $10 \mathrm{mmHg}$ at Day 1 and Day 14 of treatment. Ampreloxetine had a favorable safety profile in healthy adults and subjects with $\mathrm{nOH}$ with no serious AEs. Most common AEs in healthy subjects were dry mouth, headache, and dizziness, and in subjects with $\mathrm{nOH}$, urinary tract infection, hypertension, and headache. Overall, there were no clinically significant findings on electrocardiogram in either study population.

Conclusion: Ampreloxetine at a median dose of $10 \mathrm{mg}$ improved decrease in standing SBP in subjects with $\mathrm{nOH}$, with few cardiovascular effects in healthy subjects. In both study populations, responses at Day 1 and Day 14 of treatment were similar, suggesting no lag effect. Ampreloxetine showed the desired pressor response in subjects with $\mathrm{nOH}$ and did not produce deleterious hypertension in healthy adults.

Funding: Theravance Biopharma.

\section{Virtual Poster \#8}

Integration of palliative medicine into a multidisciplinary clinic for multiple system atrophy

E.A. Coon $^{1}$, P. Stevens ${ }^{1}$, C. Chou ${ }^{1}$, M. Suarez ${ }^{1}$, P. Sandroni ${ }^{1}$, J.H. Bower ${ }^{1}$, F. Ali ${ }^{1}$, P.A. Low ${ }^{1}$, W. Singer ${ }^{1}$, L. Rhee ${ }^{2}$

${ }^{1}$ Department of Neurology, Mayo Clinic, Rochester, MN, USA;

${ }^{2}$ Department of Palliative Medicine, Mayo Clinic, Rochester, MN, USA

Background: Multiple system atrophy (MSA) is characterized by autonomic failure and motor symptoms of predominant parkinsonism (MSA-P) or cerebellar ataxia (MSA-C). Throughout the disease course, patients with MSA develop symptoms which may benefit from a multidisciplinary team including palliative medicine. Understanding how features of the disease contribute to patient distress will impact clinical management.

Objective: To integrate palliative medicine into a multidisciplinary care model to treat patients with MSA and determine which features of the disease impact patient-reported levels of distress.

Methods: We developed a multidisciplinary clinic for patients with MSA, including Palliative Medicine, Nursing, Speech Pathology, Sleep Medicine, and Physical Medicine and Rehabilitation. We prospectively obtained Unified MSA Rating Scale (UMSARS) scores Part I and Part II; and palliative medicine instruments, the Edmonton Symptom Assessment Scale and The Distress Thermometer (graded 0-to-10; 10 represents highest distress). Statistical analyses were performed with statistical significance set at $p<0.05$. Student's t test was used for continuous variables with Chi square analysis for categorical variables.

Results: During the initial 6 months of the Mayo Clinic MSA Clinic, 22 unique patients were evaluated with 5 patients having more than 1 visit. MSA-P was the most common subtype (12 patients; $54 \%$ ) compared to MSA-C (10 patients; 36\%) while 2 patients were determined to have dementia with Lewy bodies (9\%). The majority of patients were male (12 patients; $54 \%$ ). The median time from onset of MSA symptoms to clinic visit was 5.71 years (interquartile range 3.17-8.70 years). Median UMSARS I was 20.5 (IQR 13-29.25) while UMSARS II was 19 (IQR 14.25-19). Median distress score was 4 (IQR 2-5) and women reported higher distress (5; IQR 3.5-6.5) compared to men (3; IQR 1.3-4) $(p=0.0063)$. Higher distress scores were not associated with orthostatic hypotension, urinary incontinence, catheterization or longer disease duration.

Conclusions: A multidisciplinary care model that includes palliative medicine and use of palliative care instruments may guide care for patients with MSA. Self-reported levels of distress may be higher in women with MSA; however, this data should be interpreted cautiously due to small numbers and non-validated use of instruments in the MSA population. 


\section{Virtual Poster \#9}

Association between cardiovascular autonomic control and work ability index in patients with pure autonomic failure

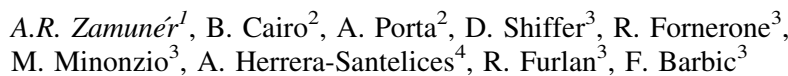
${ }^{1}$ Department of Kinesiology, Universidad Católica del Maule, Talca, Maule, Chile; ${ }^{2}$ Department of Biomedical Sciences for Health, University of Milan, Italy; ${ }^{3}$ Internal Medicine, Humanitas Clinical and Research Center-IRCCS, Humanitas University, Rozzano, Italy; ${ }^{4}$ Servicio de Medicina Física y Rehabilitación, Hospital de Curicó, Servicio de Salud del Maule, Maule, Chile

Background: Pure autonomic failure (PAF) is a rare disorder belonging to the group of synucleinopathies, characterized by autonomic nervous system degeneration. Clinical features include symptomatic orthostatic hypotension and symptoms of cerebral hypoperfusion. Symptoms usually start at middle age, thus affecting people at their working age. Therefore, elucidating whether cardiovascular autonomic control is related to the work ability in patients with PAF is relevant to propose new strategies to prevent early retirement or to extend the working life of these patients.

Objective: To evaluate the association between work ability index and cardiovascular autonomic control in patients with PAF.

Methods: Ten patients with diagnosis of PAF, $55.5 \pm 2.0$ years old, who were currently engaged in paid work took part in the study. Electrocardiogram, blood pressure and respiratory activity were continuously recorded for $10 \mathrm{~min}$ in both supine and $70^{\circ}$ head-up tilt. Spectral analysis provided the overall power spectrum density (variance of $\mathrm{RR}$ intervals, $\mathrm{VAR}_{\mathrm{RR}}$ ) and indices of cardiac parasympathetic modulation $\left(\mathrm{HF}_{\mathrm{RR}}\right.$ and $\left.\mathrm{HF}_{\mathrm{RRnu}}\right)$ and sympathetic vasomotor control $\left(\mathrm{LF}_{\mathrm{SAP}}\right)$. Delta $(\Delta)$ change from supine to standing posture was calculated in order to account for the autonomic adjustment to the orthostatic stimulus. Work ability was assessed by a self-administered questionnaire, the Work Ability Index (WAI). The scores ranged from 7 to 49 , with higher values indicating better work ability and lower values indicating unsatisfactory or jeopardized work ability. Spearman tests were applied $(\alpha=5 \%)$.

Results: Significant correlations were found between WAI and $\Delta \operatorname{VAR}_{\mathrm{RR}}(\mathrm{r}=-0.63, \mathrm{p}=0.05), \Delta \mathrm{HF}_{\mathrm{RR}}(\mathrm{r}=-0.70, \mathrm{p}=0.03)$, and $\Delta \mathrm{HF}_{\mathrm{RRnu}}(\mathrm{r}=-0.86, \mathrm{p}=0.001)$. Results indicate that the patients with better adjustment to the orthostatic stimulus, characterized by greater vagal withdrawal, were those who reported higher values of WAI.

Conclusion: Work ability is related to the cardiac autonomic adjustment to the orthostatic stimulus. Therefore, patients with greater cardiac autonomic impairment should be closely followed by their physicians and healthcare professionals in order to prevent early retirement and to extend their working age.

Funding: Supported by the Italian Ministry of Health, grant \# RF2013-02355242 and ARZ was supported by FONDECYT Iniciación grant 11180310.

\section{Virtual Poster \#10}

\section{Objective longitudinal evaluation of autonomic function in isolated REM sleep behavior disorder}

L. Baldelli ${ }^{1}$, A. Querzani ${ }^{1}$, L. Sambati ${ }^{1,2}$, G. Calandra-Buonaura ${ }^{1,2}$, P. Guaraldi ${ }^{2}$, F. Baschieri ${ }^{1,2}$, F. Provini $^{1,2}$, P. Cortelli $^{1,2}$

${ }^{1}$ Department of Biomedical and NeuroMotor Sciences (DiBiNeM), University of Bologna, Bologna, Italy; ${ }^{2}$ IRCCS Istituto delle Scienze Neurologiche di Bologna, Bologna, Italy
Objectives: To objectively assess autonomic function in a group of patients with isolated REM sleep behavior disorder (iRBD) and its longitudinal progression.

Background: Autonomic impairment is a common marker of progression towards neurodegeneration in iRBD. Several studies have reported more autonomic symptoms in iRBD compared to controls. Cardiovascular autonomic dysfunction is frequently reported in these patients, with greatest impairment in sympathetic function. However, prospective data on these markers are limited.

Methods: We enrolled consecutive videopolisomnography-confirmed iRBD patients and followed them longitudinally for a mean of $2.30 \pm 1.01$ years. Each patient underwent Ewing's battery of cardiovascular reflexes tests and the Scale for Outcomes in Parkinson's Disease-Autonomic (SCOPA-AUT). Procedures were performed at baseline, and annually. The first (T0) and the last (FU) evaluation were selected for each patient for comparison.

Results: We enrolled 18 patients (13 males-72\%), with a mean age of $68.94 \pm 6.96$ years. At T0, 8 iRBD $(47 \%)$ showed a pathologic Valsalva Maneuver (VM), of them 7 showed orthostatic hypotension $(\mathrm{OH})$ at the 3rd minute of tilting. Handgrip (HG), cold face (CF) and deep breathing (DB) test were abnormal in 6 patients each. Mean SCOPA-AUT score was $11.0 \pm 7.70$, with a greater prevalence of urinary and cardiovascular symptoms (94\% and 67\% of patients, respectively). At FU, 9 iRBD presented $\mathrm{OH}(\mathrm{p}=0.317)$ and the frequency of abnormal VM, HG, CF and DB did not significantly change. Considering blood pressure responses, diastolic blood pressure change at the 10th minute of tilt significantly increased in magnitude $(-1.78 \pm 7.73$ vs. $-6.28 \pm 12.27 \mathrm{mmHg}, \mathrm{p}=0.017)$ as did systolic change with a tendential increase $(p=0.052)$; these values did not reach statistical significance at 3rd minute. HG diastolic pressure change also decreased of $3.52 \mathrm{mmHg}(95 \% \mathrm{CI}-7.56$ to $-0.50, p=0.041$ ) in respect to T0. SCOPA-AUT score increased of 2.39 points $(95 \%$ CI $0.17-4.95 ; \mathrm{p}=0.014)$, with patients presenting one additional altered domain on average $(p=0.005)$. The greatest increase was among the sexual $(\mathrm{p}=0.023)$, thermoregulatory and urinary domains ( $\mathrm{p}=0.049$, respectively).

Conclusions: Our results confirm not only the presence of autonomic impairment in iRBD but also its gradual worsening over time justifying its value as a marker of progression of the disease.

\section{Virtual Poster \#11}

Symptoms of sympathetic dysfunction in patients with synucleinopathy referred for evaluation in a Chilean autonomic laboratory

J. Idiaquez ${ }^{1}$, J.C. Casar ${ }^{1}$, J.F. Idiaquez ${ }^{3}$, R. Iturriaga ${ }^{2}$ Departments of ${ }^{1}$ Neurology and ${ }^{2}$ Physiology, Pontificia Universidad Catolica de Chile, Santiago, Chile; ${ }^{3}$ Department of Neurology, Universidad del Desarrollo, Santiago, Chile

Aim: The major goal of this study was to describe symptoms of cardiovascular and sweating autonomic dysfunction in patients with synucleinopathies.

Patients and Methods: 73 consecutive patients, grouped in: pure autonomic failure $(\mathrm{PAF})(\mathrm{n}=10,68.6 \pm 3.6$ years $)$, multiple system atrophy (MSA) ( $=33,63.3 \pm 1.7$ years), and Parkinson's disease (PD n $=30,65 \pm 2$ years). Cardiovascular and sudomotor symptoms were evaluated with the SCOPA-autonomic score. Cardiovascular autonomic tests: blood pressure (BP) and heart rate (HR) change on standing. Valsalva maneuver. Neurogenic orthostatic hypotension (nOH) criteria: sustained systolic BP fall $\geq 20 \mathrm{mmHg}$. Sudomotor tests: sympathetic skin responses (SSR). Data Mean \pm SEM or \%. 
Results: 1. Cardiovascular: patients with nOH: PAF (100\%), MSA (87.8\%), and PD (46.7\%). Autonomic tests: systolic BP fall on standing was higher in PAF $(66.9 \pm 8.1 \mathrm{mmHg})$ related to MSA $(44.9 \pm 4.5 \mathrm{mmHg})$ and $\mathrm{PD}(15.2 \pm 3.2 \mathrm{mmHg})$. Orthostatic symptoms: dizziness + vision problems in PAF $(90 \%)$, in $\mathrm{MSA}+\mathrm{nOH}$ $(82.8 \%)$, in $\mathrm{PD}+\mathrm{NOH}(78.6 \%)$ and in $\mathrm{PD}$ without $\mathrm{nOH}(0 \%)$, syncope in PAF $(100 \%)$, in MSA $+\mathrm{nOH}(24.1 \%)$, in $\mathrm{PD}+\mathrm{nOH}$ $(14.3 \%)$ and PD without $\mathrm{nOH}(0 \%)$, weakness in PAF in $(90 \%)$, $\mathrm{MSA}+\mathrm{nOH}(69 \%)$, and in $\mathrm{PD}+\mathrm{nOH}(57.1 \%)$. SCOPA-cardio was higher in PAF $(14.6 \pm 1.0)$, related to $\mathrm{MSA}+\mathrm{nOH}(11.1 \pm 0.5)$, $\mathrm{PD}+\mathrm{nOH}(10.4 \pm 2.4)$, and PD without $\mathrm{nOH}(0.25 \pm 0.2) .2$. Sudomotor symptoms: hyperhidrosis in PAF (30\%), in MSA $(3.1 \%)$, and in PD (13.3\%). Loss of sweating in PAF (80\%), in MSA $(64 \%)$, and in PD (40\%). Segmental anhidrosis in PAF $(30 \%)$, in MSA $(0 \%)$ and in PD $(0 \%)$. Heat intolerance in PAF $(70 \%)$, in MSA (54.5\%) and in PD (37\%). Absent plantar SSR in PAF (70\%), in MSA (57.6\%) and in PD (3.3\%). SCOPA-sweating was higher in PAF $(4.0 \pm 0.9)$ and $\mathrm{MSA}+\mathrm{nOH}(5.2 \pm 0.6)$ compared to $\mathrm{PD}+\mathrm{nOH}(2.1 \pm 0.5)$ and $\mathrm{PD}$ without $\mathrm{nOH}(0.3 \pm 0.2)$.

Conclusion: PAF group showed greater cardiovascular and sudomotor symptoms than MSA and PD patients. MSA showed greater sudomotor symptoms than in PD group. $\mathrm{PD}+\mathrm{nOH}$ patients showed higher SCOPA-cardio and SCOPA-sweating scores than PD without $\mathrm{nOH}$

\section{Virtual Poster \#12}

Decreased sympathetic activity is associated with cognitive decline in Parkinson's disease

G.R. Popov ${ }^{1}$, M.A. Lebedeva ${ }^{1,3}$, D.V. Zhuravlev ${ }^{1}$, A.A. Yakovlev ${ }^{1,4}$, A.B. Guekht ${ }^{1,2}$

${ }^{1}$ Moscow Research and Clinical Center for Neuropsychiatry, Moscow, Russia; ${ }^{2}$ Pirogov Russian National Research Medical University, Moscow, Russia; ${ }^{3}$ Institute of General Pathology and Pathophysiology, Moscow, Russia; ${ }^{4}$ Institute of Higher Nervous Activity and Neurophysiology of Russian Academy of Sciences, Moscow, Russia

Introduction: Both autonomic dysfunction and cognitive decline are well known in Parkinson's disease (PD). However, the correlation between them is poorly understood. We looked at the association between quantitative autonomic and cognitive characteristics in PDpatients.

Methods: In 50 PD-patients without clinically manifest comorbidities (mean age $60.0 \pm 9.8 ; 13$ males) and 25 healthy volunteers (mean age $63.9 \pm 11.9$; 6 males), we recorded RR-intervals (RRI), beat-tobeat systolic blood pressure (BPsys), and respiratory frequency during 2 min in sitting position. We calculated parameters of total cardiac autonomic modulation (standard deviation [SD-RRI], coefficient of variation, (CV-RRI) and total powers [TP] of RRI), parasympathetic (root mean square of successive RRI differences [RMSSD], high frequency powers $[\mathrm{HF}]$ of RRI) and sympathetic cardiovascular modulation (low frequency powers [LF] of RRI and BPsys, LF/HFRRI), and baroreflex sensitivity. All patients underwent Mini-Mental State Examination (MMSE), Clock Drawing Test (CDT) and Frontal Assessment Battery (FAB). We compared autonomic parameters in PD-patients and controls using Mann-Whitney U test and evaluated association between autonomic parameters and cognitive scores in PD-patients using Spearman rank correlation (significance: $\mathrm{p}<0.05$ ). Data are presented as median and interquartile ranges.

Results: Compared to controls, PD-patients had higher respiratory frequency and lower systolic blood pressure $(113[103 ; 126]$ vs. $120[109 ; 136] \mathrm{mmHg}, \mathrm{p}=0.018)$, SD-RRI, TP-RRI, LF-RRI, and, to the greatest extent, LF-BPsys $(4.1[2.4 ; 10.3]$ vs. $10.4[5.9 ; 22.2]$ $\left.\mathrm{mmHg}^{2}, \mathrm{p}=0.001\right)$. There was significant correlation between FAB score and CV-RRI, LF-RRI, LF-BPsys, LF/HF-RRI ( $\mathrm{rs}=$ $0.310-0.366, \mathrm{p}=0.009-0.030$ ), and also between CDT score and LF/HF-RRI ( $\mathrm{rs}=0.375, \mathrm{p}=0.007$ ).

Conclusions: PD-patients demonstrated a decrease mainly in sympathetic parameters. We observed an association between sympathetic activity and cognitive level in PD-patients. These findings are in line with the concept of sympathetic denervation as a leading cause of autonomic dysfunction in PD-patients, but also might be explained by possible presence of central autonomic dysregulation.

\section{Virtual Poster \#13}

M-STAR, an ongoing Phase 3 study in participants with multiple system atrophy: baseline characteristics

J.A. Palma ${ }^{1}$, A. Ellenbogen ${ }^{2}$, R. Freeman ${ }^{3}$, G. Höglinger ${ }^{4}$ G. L'Italien', H. Kaufmann', A. Lang ${ }^{6}$, P. Low ${ }^{7}$, W. Meissner ${ }^{8}$, H. Morris ${ }^{9}$, S. Perlman ${ }^{10}$, W. Poewe ${ }^{11}$, J. Schmahmann ${ }^{12}$, W. Singer ${ }^{7}$, S. Vernino ${ }^{13}$, G. Wenning ${ }^{11}$, V. Wirtz ${ }^{5}$ I.A. Qureshi ${ }^{5}$; for the M-STAR Study Investigators

${ }^{1}$ Dysautonomia Center, New York University School of Medicine, New York, NY, USA; ${ }^{2}$ QUEST Research Institute, Farmington Hills, MI, USA; ${ }^{3}$ Center for Autonomic and Peripheral Nerve Disorders, Beth Israel Deaconess Medical Center, Boston, MA, USA;

${ }^{4}$ Department of Neurology, Hannover Medical University, Hannover, Germany; ${ }^{5}$ Biohaven Pharmaceuticals, New Haven, CT, USA; ${ }^{6}$ The Edmond J Safra Program in Parkinson's Disease University Health Network and the Department of Medicine, University of Toronto, Toronto, Ontario, Canada; ${ }^{7}$ Department of Neurology, Mayo Clinic, Rochester, MN, USA; ${ }^{8}$ University of Bordeaux CRMR Atrophie Multisystématisée CHU Bordeaux, Bordeaux, France; ${ }^{9}$ Institute of Neurology, UCL Queen Square, London, UK; ${ }^{10}$ Department of Neurology, University of California, Los Angeles, Los Angeles, CA, USA; ${ }^{11}$ Department of Neurology, Medical University of Innsbruck, Innsbruck, Austria; ${ }^{12}$ Ataxia Unit, Department of Neurology,

Massachusetts General Hospital, Boston, MA, USA; ${ }^{13}$ Department of Neurology and Neurotherapeutics, UT Southwestern Medical Center, Dallas, TX, USA

Objective: To describe baseline characteristics of participants randomized in the ongoing M-STAR phase 3 study evaluating the disease modifying effect of verdiperstat on multiple system atrophy (MSA).

Background: MSA is a rare, adult-onset, rapidly progressive, and fatal neurodegenerative disease with no disease modifying treatment available. Verdiperstat (previously BHV-3241/AZD3241) is a first-inclass, potent, selective, brain-permeable, irreversible myeloperoxidase (MPO) inhibitor. In phase 2 studies in Parkinson's disease (PD) and MSA, treatment with verdiperstat was generally safe and well tolerated. Verdiperstat decreased MPO activity in plasma, providing evidence of target engagement; reduced translocator protein binding on brain PET imaging in PD, providing proof of mechanism (decreased microglial activation/neuroinflammation) (Jucaite et al., Brain, 2015); and demonstrated favorable trends on clinical efficacy measures (Unified MSA Rating Scale [UMSARS]) at 12 weeks (Mullen et al., International MSA Congress, 2018).

Methods: M-STAR is a randomized, double-blind, placebo-controlled, parallel group study. Ambulatory participants, 40-80 years of age, with possible or probable MSA (Gilman et al., Neurology, 2008), including MSA-P or MSA-C, are randomized to 48 weeks of treatment with verdiperstat $600 \mathrm{mg}$ twice daily or placebo. Planned recruitment is approximately 250 subjects across 56 sites in 7 
countries. The primary efficacy endpoint is change from baseline to Week 48 in verdiperstat- vs. placebo-treated subjects on a score derived from the UMSARS optimized (based on health authority guidance) to assess clinically meaningful change in ability to function.

Results: M-STAR began enrolling in July 2019 and is currently ongoing; select baseline characteristics (e.g., age, gender, race, ethnicity, weight) of the initial enrollees will be presented. In addition, relevant baseline disease characteristics (e.g., disease category [MSAP vs. MSA-C]; diagnostic certainty [possible vs. probable], UMSARS Part I, Part II, and total score; Clinical and Patient Global Impression of Severity [CGI-S/PGI-S]) scales will be presented.

Conclusion: Baseline characteristics of M-STAR participants will provide key information about the enrolled population, including MSA diagnostic classifications and clinician- and patient-reported disease severities, which is significant for MSA clinical trials and therapeutic development.

Trial registration: ClinicalTrials.gov Identifier: NCT03952806. EudraCT Number: 2019-001100-38.

Funding: Study sponsored by Biohaven Pharmaceuticals (New Haven, CT).

\section{Virtual Poster \#14}

\section{Inflammatory small fiber sensory and autonomic neuropathy with prominent dysgeusia}

\section{J. Fernandez, G. Cook \\ Department of Neurology, Walter Reed National Military Medical Center, Bethesda, MD, USA}

Introduction: Peripheral taste sensation is mediated by small visceral afferent sensory fibers traveling from taste buds to the solitary nucleus. Dysgeusia is a multifactorial taste alteration characterized by an unpleasant taste. While it has been described in familial dysautonomia, it is rarely recognized in autoimmune autonomic neuropathies. Hereby, we present a case of inflammatory small fiber sensory and autonomic neuropathy (SFSAN) with prominent dysgeusia.

Case Report: A 45-year-old man presented with 4 weeks of progressive symmetric painful acral paresthesias which were followed by a persistent "awful" taste causing severe prandial nausea leading to food aversion and a $13 \mathrm{~kg}$ weight loss, a constant globus sensation causing frequent coughing spells, heartburn, constipation, erectile dysfunction, and orthostatic syncope. He took no relevant medications, had no pertinent family history or travel/occupational exposures. Neurological examination only revealed sluggish pupils with mild anisocoria, mild vibratory loss at toes, and allodynia on lower extremities. Autonomic testing revealed failure of sudomotor, cardiovagal, and sympathobaro-reflexes, and orthostatic hypotension. EMG/NCS was unremarkable. Skin biopsy showed length-dependent epidermal and sweat gland nerve fiber loss. Cerebrospinal fluid (CSF) protein was elevated to $72 \mathrm{mg} / \mathrm{dL}$. Evaluation for structural, cardiac, infectious, neoplastic, autoimmune, toxic and metabolic etiologies was unrevealing. Syncope and constipation improved with pyridostigmine. Neuropathic pain did not respond to neuro-modulatory medications. He required a gastrostomy tube for long-term nutrition given refractory upper GI symptoms to proton-pump inhibitors (PPIs). Orthostatic hypotension and sudomotor responses showed improvement after an empiric IVIg trial.

Discussion: The subacute time course and elevated CSF protein suggest an immune-mediated SFSAN. While patients with autonomic neuropathies may develop GI symptoms due to gastrointestinal dysmotility, the severe dysgeusia was deemed unique. Although gastroesophageal reflux (GR) may result in dysgeusia, the severity was considered out of proportion and did not respond to PPIs. Isolated distorted taste perception has been reported in familial dysautonomia due to partial peripheral denervation of small sensory fibers. Additionally, dysgeusia and dysautonomia have been described in antiglycine receptor encephalomyelitis. We hypothesize that the prominent dysgeusia resulted from irritation of small visceral afferent taste sensory fibers with some contribution from GR as a result of an inflammatory SFSAN.

\section{Virtual Poster \#15}

Sudomotor dysfunction in taxanes-induced peripheral neurotoxicity (CIPN) graded using the Total Neuropathy ScoreReduced in breast cancer patients

\section{S.-Y. Kim}

Department of Neurology, Ulsan University Hospital, University of Ulsan College of Medicine, Ulsan, Korea

Background: The profile of sudomotor dysfunction of taxanes-induced peripheral neuropathy has rarely been reported although substantial proportion of neuropathy showed length dependent sensory dominant axonopathy. This study measured quantitative grading of neuropathy using the reduced version of Total Neuropathy Score TNS (TNSr) and correlated this scale and sudomotor abnormality. Methods: Neuropathic symptoms were assessed using the neuropathy symptom scale. A detailed neurologic examination, nerve conduction study (NCS), and the Quantitative Sweat Measurement Test (Q-sweat test) were also performed. The TNSr score was calculated by a single examiner. We divided the patients with small fiber neuropathy and large fiber neuropathy and compared each variable between groups. Also, we analyzed correlations of the TNSr score with various parameters (NCS data, neuropathy symptom scales and Q-sweat parameters).

Results: Of 40 recruited patients, 29 (72.5\%) had large fiber neuropathy, and the other $11(27.5 \%)$ had small fiber neuropathy. Patients with large fiber neuropathy had a lower sural sensory nerve action potential (SNAP) $(p=0.000)$, lower peroneal compound muscle action potential (CMAP) $(p=0.002)$, and higher TNSr score $(p=0.000)$. There was no significant difference of Q-sweat abnormality between those groups. Only 9 of $29(31 \%)$ patients with large fiber neuropathy and 3 of $11(39 \%)$ patients with small fiber neuropathy showed a decreased sudomotor dysfunction. The TNSr score was most significantly correlated with the sural SNAP $(p=0.000)$, neuropathy symptom scale $(p=0.001)$, and pin score $(p=0.002)$ However, it was not correlated with the result of Q-sweat test.

Conclusions: The severity of taxanes-induced neuropathy using TNSr score is correlated well with NCS data and sensory symptom but not with sudomotor dysfunction. The sudomotor dysfunction did not reflect the neurotoxicity in taxanes-induced neuropathy.

\section{Virtual Poster \#16}

\section{Response to cold stress stimulus in diabetic vasculopathy: a pulse} volume study

S. Tripathi ${ }^{1}$, E. Khandelwal ${ }^{2}$

${ }^{1}$ Department of Physiology, Pt. JNM Medical College Raipur, India; ${ }^{2}$ Department of Physiology, AIIMS Raipur, India

Introduction: Diabetic autonomic neuropathy (DAN) and vasculopathy is an under diagnosed complication found in patients with 
type II diabetes mellitus (DM). Atherosclerotic changes in the vasculature lead to dysfunctional peripheral vascular dynamics and a tenfold higher risk of peripheral artery disease. Increased arterial stiffness may be an important factor linking diabetes with increased cardiovascular morbidity and mortality. Hence, measurement of vasculopathy is used to predict the development of cardiovascular disease, with sympathetic reactivity to stress being a potential marker for subsequent cardiovascular disease.

Aim and Objectives: To quantify sympathetic vascular reactivity to Cold Stress Test (CST) by using measures of photo-plethysmography (PPG) like the peak to peak interval, peak amplitude, and pulse transit time in type II DM patients and healthy subjects.

Methods: Sympathetic reactivity was assessed by finger-PPG in baseline, during CST $(1 \mathrm{~min})$ and after CST $5 \mathrm{~min}$ in 20 healthy volunteers and 20 Type II DM patients. Data obtained were compared by using statistical software for peak amplitude, pulse transit time (PTT) and peak to peak interval (PPI).

Results and Conclusion: There was no significant difference in the demographic parameters and baseline PPG parameters in the control and DM patients. Baseline heart rate was significantly higher in the patients $(p=0.009)$. A significant decrease in amplitude occurred after immersion of hand in cold water and remained low throughout CST $(p=0.006)$ and a significant slow recovery $(p=0.04)$ towards baseline after CST was seen in patients. Controls showed a significant increase in PPI between baseline and during CPT $(\mathrm{p}=0.047)$ as compared to the patients. Controls showed significant increase in PTT between baseline and during CPT $(p=0.013)$ as compared to the patients. The result of this study suggests that the collective responses of PPG amplitude, PPI and PTT can be utilized to objectively quantify the sympathetic reactivity to cold stress to detect the deficits of vascular reactivity in diabetes.

\section{Virtual Poster \#17}

\section{Does subclinical vitamin B12 deficiency alter the normal} autonomic modulation during pregnancy?

\section{S. Sharma, S. Sucharita}

Department of Physiology, St. Johns Medical College, Bangalore, India

Background: Pregnancy is associated with physiological adaptations in various organ systems including hemodynamic and cardiovascular domains. One such mechanism by which these adaptations occur is by modulations in the autonomic nervous system. In addition, deficiency of micronutrients, especially subclinical vitamin B12 deficiency, is highly prevalent during pregnancy especially among Indian women. Subclinical vitamin B12 deficiency is known to affect autonomic regulation across life cycle. The current study aimed to compare autonomic nervous activity through heart rate variability (HRV) indices during longitudinal follow up of pregnancy (across three trimesters) and also post pregnancy in women with low and high vitamin B12 status.

Methods: 50 pregnant women were longitudinally followed from trimester 1 until 2 years post birth. Lead II ECG was collected at 4 time points. i.e., each trimester and 2 years post birth, and spectral analysis was performed to obtain heart rate variability (HRV) indices. Blood samples were collected for complete hemogram and plasma vitamin B12 estimation during each trimester.

Results: Subjects were divided into low $(\mathrm{n}=24)$ and high $(\mathrm{n}=26)$ vitamin B12 groups based on vitamin B12 level of $200 \mathrm{pmol} / \mathrm{L}$. On examination of pattern of changes in HRV indices across 4-time points, all the HRV indices (low frequency (LF), high frequency (HF) and total power (TP) significantly decreased with progression of pregnancy $(\mathrm{p}<0.05)$. HRV indices measured at trimester 1 and 2 years post birth were comparable. Between subject analysis showed LF and HF HRV indices to be significantly lower $(\mathrm{p}<0.05)$ in the low vitamin B12 group across time points compared to high vitamin B12 group.

Conclusion: This study demonstrated significant reduction in autonomic activity during healthy pregnancy which increased following birth. However, subclinical vitamin B12 deficiency may impair the normal autonomic modulations associated with pregnancy.

\section{Virtual Poster \#18}

\section{Small fiber neuropathy among Gulf War Veterans evaluated at the WRIISC}

E.C. Shadiack, O. Osinubi, C. Bhate, A. Gruber-Fox, L. PatrickDeLuca, P. Cohen, D. Helmer

War Related Illness and Injury Study Center, U.S. Department of Veterans Affairs, East Orange, NJ, USA

Background: Following deployment to Operations Desert Shield/ Storm (1990-1991) (aka Gulf War), many Veterans reported developing a combination of multi-system termed Gulf War Illness (GWI). It affects between 25 and 32\% of those deployed. Prior research has identified dysfunction in the autonomic nervous system in Veterans with GWI. Some reports suggest small fiber polyneuropathy (SFPN) may underlie autonomic symptoms and chronic pain in GWI. Here, we report on a clinical program of evaluating for SFPN in Veterans with GWI.

Methods: IRB approval was obtained (VA IRB\# 01497) for this retrospective chart review of Gulf War Veterans who visited the War Related Illness and Injury Study Center (WRIISC) between 2014 and 2019, and who had a skin biopsy to evaluate for SFPN. Autonomic symptom burden was evaluated using the Composite Autonomic Severity Score 31 (COMPASS 31). COMPASS 31 scores and presence of SFPN were compared in those with and without a clinical diagnosis of GWI. Microsoft Excel and SPSS 12.0.1 for Windows were used for statistical analysis.

Results: 51 Gulf War Veterans [49 male, mean age of 48.6 years $( \pm 3.7)]$ were included. $42(82.4 \%)$ of 51 received a clinical diagnosis of GWI. $24(57.1 \%)$ of these 42 had SFPN. In GWI Veterans with SFPN, a previously known potential etiology was identified in all of them. Of GWI Veterans with SFPN, mean total COMPASS 31 score was 38.3 ( \pm 14.3 ). There was no statistical difference in COMPASS 31 scores in those with or without SFPN.

Conclusion: SFPN was frequently identified in Veterans with GWI seen at the WRIISC. Our clinical sample reported a high degree of autonomic symptom burden consistent with other published studies of patients with SFPN (14), but the SFPN cannot explain all of GWI as there was no difference in symptom burden in those with and without SFPN. The SFPN in our sample could be explained by a number of previously reported potential etiologies. Additional research is needed to further elaborate the extent and etiology of SFPN in this population, and to what extent it may be contributing to their symptoms.

\section{Virtual Poster \#19}

Activation of the rostral ventrolateral medulla results in differential increases in adrenal versus splanchnic sympathetic nerve activity

S.S. Kulkarni, N.A. Mischel, P.J. Mueller 
Department of Physiology, Wayne State University School of Medicine, Detroit, MI, USA

The sympathetic nervous system mediates the "fight-or-flight" response, including stimulation of the adrenal medulla to secrete epinephrine. Epinephrine increases heart rate and contractility and increases plasma glucose. Sympathetic nerve activity (SNA) to the adrenal gland and other organs is regulated by the rostral ventrolateral medulla (RVLM). The RVLM responds to decreases in blood pressure by increasing sympathetic nerve activity. When blood pressure decreases, RVLM activity increases via withdrawal of tonic GABAergic inhibition. Whether all sympathetic nerves rely on withdrawal of GABAergic inhibition to increase SNA is unknown. The goals of the present study were to (1) compare effects of contralateral vs. ipsilateral RVLM activation on SNAs, and (2) determine the role of RVLM $\mathrm{GABA}_{\mathrm{A}}$ receptors in hypotension-induced increases in splanchnic SNA (sSNA) and adrenal SNA (AdSNA). We hypothesized that activation of the RVLM produces differential increases in SSNA vs. AdSNA, depending on the stimulus used. In male Sprague-Dawley rats we measured AdSNA and sSNA during ipsilateral or contralateral activation of the RVLM. We produced decreases in blood pressure (sodium nitroprusside, i.v.) before and after bilateral $\mathrm{GABA}_{\mathrm{A}}$ receptor blockade (bicuculline, $5 \mathrm{mM} 90 \mathrm{~nL}$ ). Blood glucose was also recorded before and after 2-deoxyglucose (2DG, i.v.). Adrenal glands from active (12 week running wheels) and sedentary animals were analyzed to measure adrenal medulla size. We observed that glutamate $(100 \mathrm{mM}, 30 \mathrm{~nL})$ injected into the left RVLM (ipsilateral) produced increases in AdSNA that were more than twice as large as AdSNA increases to right RVLM (contralateral) injections $(\mathrm{p}<0.05 ; \mathrm{n}=6)$. In contrast, sSNA responses were equivalent to glutamate injections on either side of the RVLM. Both sSNA and AdSNA increased in response to hypotension; however, increases in sSNA but not AdSNA were eliminated by blocking $\mathrm{GABA}_{\mathrm{A}}$ receptors $(\mathrm{p}<0.05 ; \mathrm{n}=4)$. Preliminary studies indicated no differences in adrenal medulla size $(\mathrm{p}>0.05, \mathrm{n}=3)$ or blood glucose between active and sedentary animals $(p>0.05, n=4)$. Results suggest that the RVLM has predominantly ipsilateral innervation of adrenal nerves. Baroreflex-mediated increases in sSNA but not AdSNA are mediated by GABA in the RVLM. A deeper understanding of neural control of adrenal function provides insight into comorbidities between cardiovascular and metabolic disease pathologies.

\section{Virtual Poster \#20}

\section{Cerebral autoregulation associated with falls in older persons with orthostatic hypotension?}

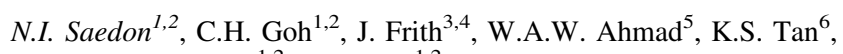
S.B. Kamaruzzaman ${ }^{1,2}$, M.P. Tan ${ }^{1,2}$

${ }^{1}$ Ageing and Age-Associated Disorders Research Group, University of Malaya, Kuala Lumpur, Malaysia; ${ }^{2}$ Division of Geriatric Medicine, Department of Medicine, Faculty of Medicine, University of Malaya, Kuala Lumpur, Malaysia; ${ }^{3}$ Falls and Syncope Service, Royal Victoria Infirmary, Newcastle Upon Tyne, UK; ${ }^{4}$ Institute for Ageing, Newcastle University, UK; ${ }^{5}$ Division of Cardiology, Faculty of Medicine, University of Malaya, Kuala Lumpur, Malaysia; ${ }^{6}$ Division Neurology, Department of Medicine, Faculty of Medicine, University of Malaya, Kuala Lumpur, Malaysia

Background: While it is considered well-established that orthostatic hypotension $(\mathrm{OH})$ is associated with falls, population-based studies have found that a large proportion of community-dwelling older adults fulfill the criteria for $\mathrm{OH}$ without experiencing falls. It is therefore postulated that older individuals with $\mathrm{OH}$ may only experience falls if cerebral autoregulation, which is the mechanism by which the brain blood flow is maintained constant through an autoregulatory range, is impaired.

Objective: To evaluate the relationship between cerebral autoregulation and $\mathrm{OH}$ in older individuals with and without a history of falls. Method: Individuals aged $\geq 60$ years were recruited from medical clinics, by word of mouth, and from existing research registries. Baseline sociodemographics, detailed medical history and medication history were obtained. Bilateral middle cerebral artery (MCA) blood flow were recorded using transcranial Doppler ultrasound (TCD) synchronized with non-invasive continuous blood pressure and ECG recordings. Synchronized brain blood flow signals, heart trace and blood pressure signals were first recorded during 10 min' supine rest. Participants were then asked to perform a standard set of mathemathical calculations, a Valsalva maneuver and to stand up from a seated position.

Results: 36 participants have been evaluated to date (13 fallers, 23 non-fallers). There is no statistically significant difference in gender, ethnicity or height and weight between groups. Fluctuations in MCA flow velocity were observed with spontaneous fluctuations in blood pressure at rest and with induced blood pressure changes during the challenge maneuvers. Visual inspection of the signals obtained suggested that changes in MCA flow velocity were more marked among fallers and non-fallers.

Conclusion: Synchronization of cerebral blood flow and continuous blood pressure signals opens up numerous new possibilities in research and clinical practice. Our preliminary findings suggest that cerebral autoregulation does play a role in determining the presence of symptomats in older individuals with $\mathrm{OH}$.

Funding: Fundamental Research Grant Scheme (FRGS) Research FRGS/1/2016/SKK02/UM/02/1.

\section{Virtual Poster \#21}

\section{Norepinephrine transporter saturation at physiological} sympathetic firing rates in the murine heart

\section{L.L. Cao ${ }^{1}$, J.M. Marshall ${ }^{1}$, L. Fabritz ${ }^{2,3}$, K.L. Brain ${ }^{1}$}

${ }^{1}$ School of Biomedical Science, Institute of Clinical Science, College of Medical and Dental Sciences, University of Birmingham, United Kingdom; ${ }^{2}$ Institute of Cardiovascular Science, College of Medical and Dental Sciences, University of Birmingham, United Kingdom; ${ }^{3}$ Department of Cardiology, University Hospitals Birmingham NHS Foundation Trust, Birmingham, UK

Introduction: Norepinephrine transporter (NET) rate is regulated by changes in NET trafficking, activity and expression. While chronic sympathetic neuron depolarisation has been consistently shown to increase NET rate, the effect of acute changes in action potential (AP) frequency has been less clear. Here, we optically measure dynamic changes of murine cardiac single-terminal NET activity ex vivo in response to firing rates over the physiological range.

Methods: Left atrial appendages (LAA) of C57BL/6 mice were isolated, loaded with fluorescent NET substrate (Neurotransmitter Transporter Uptake Assay; NTUA) and imaged with confocal microscopy, as we recently described (Cao et al., 2020). Electrical field stimulation (EFS) was delivered via two parallel electrodes (0.1-0.2 ms pulse width, $80 \%$ of cardiomyocyte contraction threshold, $0.2-10 \mathrm{~Hz}$ ) in square wave pulses to initiate APs. Competition by $\mathrm{NE}$ at NET was assessed by exogenous NE application during the NTUA wash-out phase. ' $n_{\mathrm{t}}$ ': individual nerve terminals; data were compared by Kruskal-Wallis test and post hoc analysis.

Results: In the first 3 min of EFS, NTUA uptake was progressively suppressed in response to increasing EFS frequencies in the mouse 
LAA. Thus, median (IQR) NET reuptake rate in the absence of stimulation [15.8 (8.0-23.5) \%. $\left.\mathrm{min}^{-1} ;\left(\mathrm{n}=7, \mathrm{n}_{\mathrm{t}}=90\right)\right]$ was reduced by $61 \%$ at $2 \mathrm{~Hz}\left[6.2(0.6-20.3) \% \cdot \mathrm{min}^{-1}\right], 66 \%$ at $5 \mathrm{~Hz}[5.3(-1.6$ to $15.3) \% \cdot \mathrm{min}^{-1}$ ] and $72 \%$ at $10 \mathrm{~Hz}\left[4.4(-4.7\right.$ to 14.0$\left.) \% \cdot \mathrm{min}^{-1}\right]$ (all stimulation groups: $\left.\mathrm{n}=4, \mathrm{n}_{\mathrm{t}}=60 ; p<0.01\right)$. In the absence of EFS, exogenously applied NE $(1-100 \mu \mathrm{M})$ during wash-out significantly accelerated NTUA displacement median (IQR) rate in a concentration-dependent manner compared to control $[-6.6$ ( -16.7 to $6.1) \% \cdot \mathrm{min}^{-1}$ ], for example, by over two-fold in the presence of $1 \mu \mathrm{M}$ $\left[-24.9(-45.0\right.$ to -16.8$\left.) \% \cdot \mathrm{min}^{-1}\right]$ (all groups: $\mathrm{n}=4, \mathrm{n}_{\mathrm{t}}=60$; $p<0.0001)$.

Conclusion: In the heart, acute increases in sympathetic firing rate progressively saturate single-terminal NET reuptake rate. This implies a supralinear relationship between firing frequencies and extracellular NE concentrations, with implications for cardiac overexcitability and arrhythmogenesis. Furthermore, substrate competition of $\mathrm{NE}$ at NET also has implications for the clinical interpretation of ${ }^{123}$ I-MIBG cardiac scans under conditions of varying sympathetic tone.

Funding: This work was supported by the British Heart Foundation studentship [grant number FS/17/7/32651 to LLC], British Heart Accelerator Award [AA/18/2/3421 to ICVS (LF)] and Leducq Foundation via ICVS.

\section{Virtual Poster \#22}

\section{Direct recording of rat vagal tone}

\section{J.T. Marmerstein, G.A. McCallum, D.M. Durand}

Biomedical Engineering, Case Western Reserve University, Cleveland, $\mathrm{OH}$, USA

"Vagal tone" is a clinical measure presumed by some to indicate overall levels of vagal activity. Low vagal tone has been associated with many severe conditions such as diabetes, heart failure and hypertension, yet has so far only been measured indirectly, commonly through the heart rate variability (HRV). It has been hypothesized that vagal nerve stimulation (VNS) therapies could be used to fix the imbalance of vagal tone associated with these diseases, and VNS has seen widespread use, most famously for the treatment of drug-resistant epilepsy. Despite the effectiveness of VNS in some cases, others have seen minimal success, including three recent studies which explored VNS for treatment of heart failure (ANTHEM-HF, INOVATE-HF, and NECTAR-HF) but found little change in patient endpoints. Elucidating the relationship between vagal activity and HRV may establish a better understanding of vagal physiology, which can be used to better understand VNS mechanisms, stimulation parameters, and targets, thus improving therapy outcomes. We have developed a methodology to directly measure vagal activity chronically in awake, behaving animals, allowing, for the first time, true measures of vagal tone. Electrodes made of carbon nanotube yarns (CNTY) were implanted inside the left cervical vagus of rats, along with ECG electrodes for simultaneous measurement of HRV. Tonic vagal activity was recorded in eight animals, with and without isoflurane anesthesia. Vagal activity was averaged over 5-10 min periods and compared to common HRV metrics used for vagal tone, including time and frequency domain measurements. While the name "vagal tone" implies a tonic level of baseline activity in the vagus nerve, our results show that average neural activity does not correlate with any of the HRV metrics analyzed. Isoflurane is known to alter HRV and individual fiber behavior, but even unanesthetized animals did not exhibit a significant correlation between HRV and true vagal tone. Overall these results suggest that "vagal tone" is a misleading term, and confirms that HRV is likely representative of a small subset of vagal fibers, rather than overall vagal activity.

Funding: Congressionally Directed Medical Research Programs, Autonomic nervous system activity and the implications on breast cancer metastasis (W81XWH-18-1-058); National Institutes of Health, Nerve reshaping for improved selectivity (5R01NS03284522).

\section{Virtual Poster \#23}

\section{Vagal reactivation after stress testing is associated with vagal deactivation during orthostatic testing in women in the luteal phase of the menstrual cycle}

G.M.R. Cunha, E.M.K.K. Soares, C.J.G. da Cruz, L.G.G. Porto, D.S. Martin, M. Morlim, G.E. Molina

Exercise Physiology Laboratory, Faculty of Physical Education, University of Brasília, Brazil

Introduction: Low cardiac autonomic function (CAF) modulation is strongly associated with increased cardiovascular events, including sudden death and all-cause mortality in the general population. At rest, CAF can be evaluated over the active orthostatic stress test (AOS) and also after a maximal exercise stress test by heart rate variability (HRV). Both methods are considered valid and reproducible to assess the degree of responsiveness of parasympathetic activity under different clinical and functional conditions. It is not yet clear if the parasympathetic reactivation immediately after maximal exercise stress test is associated with the parasympathetic deactivation during the initial moments of AOS. Most studies using HRV delimit their samples to male volunteers. Therefore, more studies with females are necessary due to the autonomic differences between them. The present study aimed to analyze the correlation between parasympathetic deactivation- $\mathrm{RR}$ interval response (RR) during AOS - and parasympathetic reactivation (HRV following a maximal exercise test) in healthy women in the early stage of the menstrual cycle.

Method: Ten healthy women $(\mathrm{n}=10)$ aged $20 \pm 1.5$ years and BMI $23.0 \pm 2.7 \mathrm{~kg} / \mathrm{m}^{2}$ in the luteal phase of the menstrual cycle (between 19 th to 21 st day of regular 28-day cycles) were evaluated. Blood pressure, respiratory rate was collected. The RR intervals were recorded throughout the AOS (first $6 \mathrm{~s}$ ) at rest and following a maximal exercise stress test-recovery phase $(2.5 \mathrm{~km} / \mathrm{h} 2.5 \%)$ until the 5 th min of recovery-using the Polar RS $800 \mathrm{cx}^{\circledR}$ frequency meter. Subsequently, the data were analyzed and processed using the Kubios $\mathrm{HRV}^{\mathrm{TM}}$ time-domain (rMSSD) and Poincaré (SD1) domains, both parasympathetic activity measurements. Due to the data distribution, nonparametric statistics were used. Significance was set at $5 \%$ (Spearman correlation).

Results: There was a positive correlation between parasympathetic reactivation in the initial moments of the active recovery phase $(30 \mathrm{~s}$ and $1 \mathrm{~min}$ ) with parasympathetic deactivation during AOS (rMSSD $\mathrm{rs}=0.52 ; 0.55, \mathrm{p}=0.04 ; 0.05$ and SD1 $\mathrm{rs}=0.53 ; 0.55, \mathrm{p}=0.05$; $0.05)$. No significant correlations were observed between the later minutes of parasympathetic reactivation (3rd and 5th minute of active recovery).

Conclusion: Parasympathetic reactivation in the initial moments of recovery (the $30 \mathrm{~s}$ and $1 \mathrm{~min}$ ) after a maximal effort is positively correlated with parasympathetic deactivation during AOS in healthy women with regular menstrual cycle who were evaluated during their luteal phase of the menstrual cycle. 


\section{Virtual Poster \#24}

High fat diet-induced adaptations in cardiac vagal motor neuron electrophysiology in the pathogenesis of cardiovascular disease

\section{Espinoza, C.R. Boychuk}

Department of Cellular and Integrative Physiology, University of Texas Health San Antonio, San Antonio, TX, USA

The link between consumption of fat and the development of cardiovascular disease (CVD) has long been established. Still, the obesity-independent mechanisms through which a high fat diet (HFD) contributes to the pathogenesis of CVD remains elusive. Increasing evidence has linked autonomic dysfunction-characterized by a potentiation of sympathetic activity, and a decrease in parasympathetic vagal tone-to a broad spectrum of CVDs. Parasympathetic vagal innervation to the sinoatrial node originates from brainstem neurons located in the nucleus ambiguus and the dorsal motor nucleus of the vagus (DMV). However, aside from the therapeutic potential of DMV optogenetic activation in models of CVD, little is known about their electrophysiological properties, or how these are altered by HFD exposure. In the present study, we investigated the obesity-independent effect of short-term HFD consumption on the electrophysiological properties of cardiac motor neurons in the context of decreased parasympathetic tone. We hypothesized that HFD exposure would modulate the electrophysiological properties of cardiac projecting motor neurons. These adaptations, we believe, can either exacerbate into cardiac manifestations if left unaltered, or be harnessed as a potential therapeutic target. Mice were challenged with HFD or control chow for 2 weeks. During this time, we employed in vivo heart rate telemetry to monitor HFD-induced alterations in heart rate (HR). We found that HFD-fed mice exhibited significant increases in HR at day 3 of HFD exposure $(p=0.0159)$, with no significant differences during the second week. We next investigated whether the lack of differences in HR during the second week of HFD exposure were the result of a compensatory process by determining HFD-induced modulations in the signaling properties of cardiac motor neurons. Mice underwent retrograde tracing to identify cardiac motor neurons, followed by in vitro whole-cell patch-clamp electrophysiology. Under the current clamp configuration, we found that despite no differences in firing frequency, cardiac DMV motor neurons from HFD-fed mice showed a significant increase in evoked action potentials following GABAergic blockade $(\mathrm{p}<0.0001)$. Our findings suggest that increased sensitivity to GABAergic blockade might represent an adaptive response to HFD exposure, which can account for the similarities in HR at week two of HFD exposure. Funding: American Heart Association (AHA) 20PRE35180105 and NIH Initiative for Maximizing student development grant R25GM095480-08 to LE; AHA16SDG26590000 and UT Health Start-up to CRB.

\section{Virtual Poster \#25}

\section{Evidence for signaling plasticity in preganglionic} parasympathetic motor neurons in the dorsal motor nucleus of the vagus as a contributor to the regulation of pancreatic alpha cell regulation

S. Fedorchak, M. Lopez, T. Fujikawa, C.R. Boychuk Department of Cellular and Integrative Physiology, University of Texas Health Science Center at San Antonio, San Antonio, TX, USA

During hypoglycemic challenge, alpha cells within the pancreas release glucagon to facilitate the release and production of glucose from hepatic stores. Although current dogma indicates that intrapancreatic signaling primarily mediates alpha cell-dependent glucagon release, a significant body of evidence implies the autonomic nervous system as an additional regulator of pancreatic secretions, specifically via the dorsal vagal motor nucleus (DMV). However, the role of the DMV in glucagon regulation is understudied. Therefore, this study sought to provide evidence using electrophysiology and retrograde tracing that the DMV plays a role in maintaining glucose homeostasis during hypoglycemic challenge. Based on previous literature identifying increased vagal nerve activity associated with glucagon release, we hypothesized that fasting would increase excitable neurotransmission to DMV neurons. Brainstem tissue containing the DMV was collected from mice for whole cell patchclamping immediately following an overnight $16-\mathrm{h}$ fast. Fasting significantly decreased the frequency of excitatory post-synaptic currents $(1.96 \pm 0.34 \mathrm{HZ}, \mathrm{n}=2)$ in DMV motor neurons compared to DMV neurons from fed mice $(10.74 \pm 0.82 \mathrm{HZ}, \mathrm{n}=4)$, suggesting that, contrary to our hypothesis, fasting suppresses DMV excitatory neurotransmission. To identify if this reduced excitatory neurotransmission could be translated to the pancreas via DMV neurons, initial experiments injected non-toxic retrograde tracers directly into the pancreas and determined that DMV neurons can be directly labelled $(9.0 \pm 4.0$ DMV neurons; $n=2)$. To determine if DMV neurons projected to alpha cells, we used a Cre-dependent pseudo-rabies virus (PRV) injected into the pancreas of mice which expressed Cre in pancreatic alpha cells $(\mathrm{Gcg}-\mathrm{ER} 2-\mathrm{Cre} ; \mathrm{n}=3)$. These experiments confirmed that DMV neurons were labeled, which suggests that DMV neurons are part of a pathway which may regulate pancreatic alpha cells (34.7 \pm 6.6 labelled DMV neurons). Taken together, these data suggest that synaptic signaling to DMV neurons is altered during a 16-h fasting period and DMV neurons do send innervations to pancreatic alpha cells. This study also provides compelling evidence that inhibition (not activation) of vagal efferent activity is related to hypoglycemic challenge, and likely the release of glucagon. Future studies will continue to directly investigate how changes in synaptic signaling in the DMV after fasting may translate to alpha cell glucagon secretion.

Funding: AHA 16SDG26590000 to CRB; T32 NS082145 to SF.

\section{Virtual Poster \#26}

\section{Cardiovascular deconditioning induces synaptic and neuronal plasticity in the nucleus tractus solitarii of rats}

\section{Lima-Silveira, D. Martinez, E.M. Hasser, D.D. Kline}

Department of Biomedical Sciences and Dalton Cardiovascular Research Center, University of Missouri, Columbia, MO, USA

The hindlimb unloading (HU) rat model induces cardiovascular deconditioning (CVD) analogous to that observed in individuals exposed to microgravity or bed rest. HU rats have increased resting heart rate, elevated blood pressure and impaired baroreflex function. The nucleus tractus solitarii (nTS) is a critical central region where autonomic and cardiovascular reflex function are integrated. In this study we tested the hypothesis that CVD following HU modulates glutamatergic neurotransmission and neuronal properties of nTS neurons. For this purpose, stainless wire rings were placed in the tail of male Sprague-Dawley rats and suspended at $30-35^{\circ}$ for 2 weeks with free access to food and water. Control animals were maintained in normal postural conditions. After the HU protocol, coronal brainstem slices that contain nTS were generated. The whole-cell patch clamp technique and live-cell calcium imaging were performed to evaluate in monosynaptic neurons glutamatergic excitatory post synaptic currents (EPSCs), properties of nTS neurons, and calcium 
signaling in visceral afferents. In response to afferent tractus solitarii stimulus, the amplitude of EPSCs and influx of calcium into afferent terminals was greater in HU than controls. Spontaneous EPSCs were comparable between groups. Despite the increase in evoked glutamatergic signaling, HU neurons were more hyperpolarized and exhibited increased rheobase, depolarized threshold, and decreased excitability compared to controls. The decreased excitability and hyperpolarized membrane was associated with enhanced GABAergic modulation to these neurons. The results indicate that HU induces both synaptic and neuronal plasticity in nTS. Altogether these alterations may contribute to the basal autonomic and reflex effects of cardiovascular deconditioning following HU.

Funding: NIH, HL132836, AAS-Lundbeck fellowship.

\section{Virtual Poster \#27}

\section{Control of heart function by nucleus ambiguus neurons}

T.C. Coverdell, R.-J. Abraham-Fan, N.M. Schiavone, M. Crook, S.B. Abbott, J.N. Campbell

Department of Pharmacology, University of Virginia, Charlottesville, VA, USA

Heart rate is a well-established indicator of overall health and wellbeing, yet little is known about the neurons that control it. Although the heart has its own pacemaker independent of the brain, heart rate is largely regulated by the autonomic nervous system, subjecting it to the interplay between sympathetic activities (via sympathoexcitatory neurons) and parasympathetic activities (via cardiac vagal preganglionic neurons (CVNs)). Studies have also suggested that CVNs control a wide variety of cardiac functions, ranging from heart rate and atrioventricular conductance (via nodal tissue) to contractility and excitability (via ventricular myocardium). The nucleus ambiguus (nAmb), a region in the medullary reticular formation of the brainstem, is a major center of parasympathetic cardiac control and houses the majority of CVNs. nAmb neurons are also known to control respiratory functions (bronchoconstriction, bronchosecretion) and upper airway and esophageal muscles, making it challenging to isolate CVNs for study. This has greatly limited what we know about their gene expression, synaptic circuitry, and specific roles in cardiac function. To address these issues, we characterized cardiovagal neurons based on transcriptome-wide mRNA expression, synaptic circuitry, and sufficiency for heart rate control. Our single-cell transcriptomics analysis pointed to three molecularly distinct neuron subtypes (marked by the genes Crhr2, Vipr2, and Tbx3) localized in the nAmb, which we confirmed and anatomically mapped by in situ detection of marker transcripts. We profiled the projections of Tbx3 $\mathrm{nAmb}$ neurons by injecting a Cre-dependent reporter vector into the nAmb of Tbx3-CreER mice, followed by tamoxifen administration and recovery of the heart for clearing and imaging. Our results show that these neurons innervate multiple sites in the heart. Furthermore, using an intersectional optogenetics approach to specifically manipulate the activity of nAmb neuron subtypes in vivo, we have determined that activating the nAmb subtype marked by Tbx 3 dramatically decreases heart rate. Together, these studies reveal a close relationship between the molecular and functional organization of the nAmb.

Funding: Pharmacological Sciences Training Grant (NIH: 2T32GM007055-44).

\section{Virtual Poster \#28}

Deep breathing impacts blood pressure to a greater degree in individuals with cervical spinal cord injury than able-bodied controls

M.T Maher ${ }^{1,3}$, J.P. Weir ${ }^{1,4}$, W.A. Bauman ${ }^{1,2}$, J.M. Wecht ${ }^{1,2}$

${ }^{1}$ James J Peters VA Medical Center, Bronx, NY, USA; ${ }^{2}$ Icahn School of Medicine at Mount Sinai, New York, NY, USA; ${ }^{3}$ Kessler Institute for Rehabilitation, West Orange, NJ, USA; ${ }^{4}$ University of Kansas, Lawrence, KS, USA

Background: Due to impaired descending cortical control of sympathetic vasomotor tone, individuals with cervical spinal cord injury (SCI) are often hypotensive and cannot adequately regulate blood pressure (BP) during routine activities. As a result, we speculate that changes in intrathoracic volume (intV) during deep breathing (DB) may have a heightened influence on BP compared to healthy controls. Methods: The participants included 10 individuals with SCI and 10 age-matched controls. Participants with SCI had cervical injuries (C4-C6), were predominantly motor-complete (AIS A and B) and were chronically injured (2-31 years). Participants performed the DB test, which consisted of breathing at a rate of 6 breaths $/ \mathrm{min}$ for 8 inspiratory/expiratory cycles while seated. A bioamplifier system was used to record ECG and estimate changes in intV (via impedance analysis), with simultaneous beat-to-beat BP recorded using photoplethysmography at the finger. The peak cross correlation (peakCC) between intV and BP was calculated for each 8-breath cycle during $\mathrm{DB}$ and normal breathing (NB), for the period when respiration was leading BP. Data were analyzed with ANOVA to determine significant main and interaction effects for condition (DB, NB) and group (SCI, controls) in peakCC.

Results: Although average HR did not differ significantly between the SCI and control groups ( $66 \pm 15$ vs. $65 \pm 10 \mathrm{bpm}$, respectively), $\mathrm{SBP}(86 \pm 27$ vs. $112 \pm 14 \mathrm{mmHg}$, respectively; $\mathrm{p}<0.05)$ and DBP $(54 \pm 14$ vs. $75 \pm 8 \mathrm{mmHg}$, respectively; $\mathrm{p}<0.05)$ were significantly lower in the SCI group. The interaction effect for peakCC was not significant $(\mathrm{F}=2.95 ; \mathrm{p}=0.10)$; however, the main effect for condition was significant $(\mathrm{F}=4.64 ; \mathrm{p}<0.045)$. Within group change in peakCC appeared larger in the SCI $(0.24 \pm 0.21$; Cohen's $\mathrm{d}=1.13$ ), than in the control group (change $=-0.03 \pm 0.39$, Cohen's $\mathrm{d}=-0.08)$.

Conclusion: These findings suggest that changes in respiration rate and intV during DB differentially impact blood pressure in individuals with cervical SCI as compared to controls. While the small sample size limited our ability to detect an interaction effect, the heightened influence of intrathoracic pressure fluctuations on cardiovascular hemodynamics may confound use of the DB test to assess autonomic cardiovascular control in individuals with cervical SCI. Funding: This work is supported by VA RR\&D Service (Grants B9212-C B2020-C).

\section{Virtual Poster \#29}

Impact of angiotensin receptor neprilysin inhibitors on blood pressure variability in heart failure

S. Nathaniel ${ }^{1}$, S. McGinty ${ }^{1}$, D.G. Edwards ${ }^{1}$, W.B. Farquhar $^{1}$, M.A.H. Witman $^{1}$, V. Hosmane ${ }^{2}$, M.M. Wenner ${ }^{1}$

${ }^{1}$ University of Delaware, Newark, DE, USA; ${ }^{2}$ Hosmane Cardiology, Newark, DE, USA

Introduction: One of the main benefits of angiotensin receptor neprilysin inhibitors (ARNi) in heart failure patients with reduced 
ejection fraction (HFrEF) is decreasing resting blood pressure (BP). The degree to which BP fluctuates during day and night (BP variability; BPV) is a prognostic marker in HFrEF, yet it is unclear if ARNi improves BPV. The purpose of the study was to test the hypothesis that BPV would be attenuated after 12 weeks of ARNi in HFrEF.

Methods: 9 HFrEF participants [64 \pm 10 years, EF: $29 \pm 6 \%$ ] underwent 24-h ambulatory BP measurement at baseline and following 12 weeks of ARNi therapy; BPV assessed by average real variability (ARV) and standard deviation (SD) of systolic BP (SBP) and diastolic BP (DBP).

Results: Twenty-four-hour ambulatory $\quad$ SBP $\quad(128 \pm 27 \quad$ vs. $115 \pm 14 \mathrm{mmHg} ; \mathrm{P}=0.05)$ and DBP $(77 \pm 14$ vs. $68 \pm 8 \mathrm{mmHg}$; $\mathrm{P}=0.01$ ) were reduced after 12 weeks of ARNi. During daytime SBP $(129 \pm 24$ vs. $120 \pm 19 \mathrm{mmHg} ; \mathrm{P}=0.01)$ was reduced and $\mathrm{DBP}$ $(78 \pm 13$ vs. $73 \pm 13 \mathrm{mmHg} ; \mathrm{P}=0.07)$ tended to reduce after 12 weeks of ARNi. During nighttime, SBP (125 \pm 33 vs. $110 \pm 16 \mathrm{mmHg} ; \mathrm{P}=0.19)$ and $\mathrm{DBP}(71 \pm 17$ vs. $62 \pm 11 \mathrm{mmHg}$; $\mathrm{P}=0.12$ ) were unchanged after 12 weeks of ARNi. Overall SBP ARV $(13 \pm 2$ vs. $11 \pm 2 ; \mathrm{P}<0.01)$ and $\mathrm{DBP}$ ARV $(12 \pm 2$ vs. $11 \pm 2 ; \mathrm{P}=0.05)$ were reduced after 12 weeks of ARNi. During daytime, SBP ARV (14 \pm 2 vs. $12 \pm 2 ; \mathrm{P}=0.11)$ and DBP ARV $(13 \pm 2$ vs. $12 \pm 3 ; \mathrm{P}=0.10)$ were unchanged after 12 weeks of ARNi. During nighttime, SBP ARV (12 \pm 3 vs. $10 \pm 3$; P = 0.02) was reduced and DBP ARV $(12 \pm 5$ vs. $8 \pm 3 ; \mathrm{P}=0.08)$ tended to reduce after 12 weeks of ARNi. Overall, SBP SD (20 \pm 4 vs. $16 \pm 9$; $\mathrm{P}=0.12)$ was unchanged and DBP SD $(21 \pm 4$ vs. $15 \pm 6 ; \mathrm{P}=0.03)$ was reduced after 12 weeks of ARNi. During daytime, SBP SD $(21 \pm 4$ vs. $16 \pm 7 ; \mathrm{P}=0.10)$ was unchanged and DBP SD $(21 \pm 5$ vs. $15 \pm 4$; $\mathrm{P}=0.02)$ was reduced after 12 weeks of ARNi. During nighttime, SBP SD ( $15 \pm 8$ vs. $14 \pm 8 ; \mathrm{P}=0.64)$ was unchanged and DBP SD $(15 \pm 7$ vs. $9 \pm 4 ; \mathrm{P}<0.01)$ was reduced after 12 weeks of ARNi.

Conclusion: Preliminary data suggest ARNi reduces BPV in HFrEF. Additional data are needed to understand the impact of ARNi on BPV in HFrEF.

Funding: Supported by NIH GRANT P20 GM 103446; ACSM grant 19-00934.

\section{Virtual Poster \#30}

\section{Assessment of endothelial dysfunction in sickle cell disease: an insight into vaso-occlusive crisis}

\section{E. Khandelwal ${ }^{1}$, M. Kumar ${ }^{1}$, S. Tripathi ${ }^{2}$}

${ }^{1}$ Department of Physiology, AIIMS Raipur, India; ${ }^{2}$ Department of Physiology, Pt. JNM Medical College Raipur, India

Introduction: Sickle cell disease (SCD) is a monogenic disorder of the beta globin chain characterised by the formation of Sickle haemoglobin ( $\mathrm{HbS}$ ). Polymerization of deoxy $\mathrm{HbS}$ leads to formation of tactoid within the RBCs causing a distorted sickle shape which eventually leads to increased haemolysis and vaso-occlusion crisis (VOC). Role of endothelial dysfunction and noxious microenvironment due to pro-inflammatory and pro-oxidative factors in pathophysiology of VOC is not well addressed, which is the major cause of morbidity and mortality globally. The purpose of this study is to establish the role of endothelial dysfunction in pathophysiology of vaso-occlusive crises with induced vasoconstriction which may play a role in triggering crises in patients of SCD.

Aim \& Objectives: To assess and quantify endothelial dysfunction by reactive hyperemia $(\mathrm{RH})$ in adult patients of sickle cell anaemia. To compare the vascular reactivity parameter obtained between healthy subjects and SCD patients.
Methods: A total of 30 patients of SCD were assessed for heart rate variability (HRV) and pulse wave analysis (PWA) before and after RH. Data obtained were compared with aged-matched controls using statistical software for time domain, frequency domain and non linear analysis for HRV. Peak amplitude, pulse transit Time (PTT), upstroke slope and peak to peak interval for PWA.

Result \& Conclusion: There is no significant difference in the baseline, demographic parameters in the control and SCD patient groups. Their baseline heart rate variability and PWA parameters are comparable, suggestive of normal resting functions of cardiovascular system. PWA parameters like peak amplitude, PTT, upstroke slope and peak to peak interval showed significant change in the control group after RH $(\mathrm{p}>0.005)$ compared to the SCD patient group, suggestive of an effective metabolic response in the control group. PTT response reflects the myogenic component in the early part of RH and PPG amplitude response reflects the metabolic component reinforcing the latter course of RH. Together, they are very useful to predict endothelial dysfunction and can be used clinically to assess the risk of a vaso-occlusive crisis happening in sickle cell patients, thus improving their quality of life.

Funding: Indian Council Of Medical Research (ICMR).

\section{Virtual Poster \#31}

\section{Respiratory neuroplasticity to acute intermittent hypercapnic hypoxia in humans}

\section{C.V. Brown, T.D. Vermeulen, B.M. Shafer, T.J.R. Stuckless, G.E.} Foster

Centre for Heart, Lung, and Vascular Health, School of Health and Exercise Science, University of British Columbia, Okanagan,

Kelowna, BC, Canada

Intermittent hypercapnic hypoxia (IHH) leads to respiratory neuroplasticity including increased ventilatory responsiveness to chemostimuli (progressive augmentation, PA) and increased minute ventilation $\left(\dot{\mathrm{V}}_{\mathrm{I}}\right.$; long term facilitation, $\left.\mathrm{vLTF}\right)$. The development of respiratory neuroplasticity during $\mathrm{IHH}$ depends on the hypoxic stimulus profile and may require the addition of hypercapnia. To determine if acute IHH induces PA and VLTF in healthy, young participants, we retrospectively analyzed three independent studies conducted in our laboratory. Participants $(\mathrm{N}=33 ; \mathrm{F}=6)$ were exposed to 40 cycles IHH comprised of 40-s hypercapnic hypoxia (nadir end-tidal $\mathrm{O}_{2}, \mathrm{P}_{\mathrm{ET}} \mathrm{O}_{2}: 52 \mathrm{mmHg}\{5253\}$, change in end-tidal $\left.\mathrm{CO}_{2}\left(\mathrm{P}_{\mathrm{ET}} \mathrm{CO}_{2}\right)=+4 \mathrm{mmHg}\left\{\begin{array}{ll}3 & 4\end{array}\right\}\right)$, and 20 -seconds normoxia (means $\{95 \%$ Confidence Interval $\}$ ). Tidal volume $\left(\mathrm{V}_{\mathrm{T}}\right)$, breathing frequency $\left(f_{\mathrm{B}}\right), \dot{\mathrm{V}}_{\mathrm{I}}, \mathrm{P}_{\mathrm{ET}} \mathrm{O}_{2}$, and $\mathrm{P}_{\mathrm{ET}} \mathrm{CO}_{2}$ were measured continuously throughout IHH. Data were linearly interpolated on a second-bysecond basis and signal-averaged into 10-min bins. Nadir $\dot{\mathrm{V}}_{\mathrm{I}}$ increased continuously throughout the 40-min exposure (1-10: 21 1/ $\min \{1923\}, 31-40: 231 / \min \{2126\} ; \mathrm{P}<0.001)$. The change in $\dot{\mathrm{V}}_{\mathrm{I}}$ within each 10-min bin was greater during the last $10 \mathrm{~min}$ of IHH compared with the first $10 \mathrm{~min}$ (1-10: 14 1/min \{11 18\}, 31-40: 16 1/ $\min \{1319\} ; \mathrm{P}<0.01)$. Peak $\dot{\mathrm{V}}_{\mathrm{I}}$ was greater during the last $10 \mathrm{~min}$ of IHH compared with the first $10 \mathrm{~min}$ (1-10: 35 1/min $\{3040\}, 31-40$ : 38 1/min $\{3343\} ; \mathrm{P}<0.001)$. The heightened ventilatory response to hypoxia induced during IHH is primarily driven by increases in $\Delta \mathrm{V}_{\mathrm{T}}$ (1-10: $0.81\{0.61 .0\}, 31-40: 0.91\{0.81 .1\} ; \mathrm{P}<0.001)$ while the increase in nadir $\dot{\mathrm{V}}_{\mathrm{I}}$ was primarily driven by $f_{\mathrm{B}}(1-10: 13 \mathrm{bpm}\{11$ 14\}, 31-40: $14 \mathrm{bpm}\{1316\} ; \mathrm{P}<0.001)$. These data suggest that acute IHH involving rapid cycles of hypercapnic hypoxia can induce respiratory neuroplasticity in humans as evidenced by (1) the progressive increase of the nadir $\dot{\mathrm{V}}_{\mathrm{I}}$ across the 40-min of IHH (i.e., 
vLTF), and (2) both an increased peak $\dot{\mathrm{V}}_{\mathrm{I}}$ and a greater change in $\dot{\mathrm{V}}_{\mathrm{I}}$ during each cycle of IHH (i.e., PA).

Funding: NSERC, HSFC, MSFHR.

\section{Virtual Poster \#32}

\section{Orthostatic tachycardia and functional cardiac response following strict head-down-tilt bedrest}

\section{F. Hoffmann ${ }^{1,2}$, J. Rabineau ${ }^{3}$, D. Mehrkens ${ }^{2}$, B.W. Johannes ${ }^{1}$, E. Caiani ${ }^{4}$, P.-F. Migeotte ${ }^{3}$, J. Jordan ${ }^{5}$, J. Tank ${ }^{2}$ \\ ${ }^{1}$ Institute of Aerospace Medicine, German Aerospace Center, Cologne, Germany; ${ }^{2}$ Department of Internal Medicine III, University of Cologne, Cologne, Germany; ${ }^{3}$ LPHYS, Université Libre de Bruxelles, Bruxelles, Belgium; ${ }^{4}$ Dipartimento di Elettronica, Informazione e Bioingegneria, Politecnico di Milano, Italy; ${ }^{5}$ Chair of Aerospace Medicine, University of Cologne, Cologne, Germany}

Background: Reduced physical activity and poor cardiovascular fitness predispose to orthostatic tachycardia. Functional and structural changes in the heart have been implicated. Myocardial strain measurements capture subtle abnormalities in myocardial function. We applied the approach to test the hypothesis that bedrest deconditioning produces cardiac dysfunction in healthy persons.

Materials and methods: In the AGBRESA study, which assessed artificial gravity through centrifugation as potential countermeasure for space travel, 24 healthy persons ( 8 women) were submitted to 60 days strict $-6^{\circ}$-head-down-tilt bedrest. During bedrest, eight subjects each were included in a control group or groups subjected to continuous artificial gravity training on a short-arm centrifuge [30 $\mathrm{min} /$ day] or intermittent centrifugation $[6 \times 5 \mathrm{~min} /$ day]. We assessed cardiac morphology, function, strain and hemodynamics by cardiac-MRI (baseline, end of bedrest, recovery) and echocardiography (baseline, end of bedrest). Before and after bedrest, we assessed orthostatic heart rate responses as measure of cardiovascular deconditioning.

Results: We conducted a pooled analysis because cardiovascular responses to bedrest did not differ between groups. Supine heart rate (baseline: $64 \pm 9.6 \mathrm{bpm}$; bedrest: $72.3 \pm 10.5 \mathrm{bpm}$; recovery: $69.6 \pm 10.5 \mathrm{bpm}, \quad p<0.0001)$ and diastolic blood-pressure $(69.6 \pm 7.3 \mathrm{mmHg}$; bedrest: $78.5 \pm 6.9 \mathrm{mmHg}$; recovery: $70.3 \pm 6.3 \mathrm{mmHg}, p<0.0001)$ increased with bedrest. With head-up tilt, heart rate increased $22.8 \pm 10.5 \mathrm{bpm}$ before and $45.9 \pm 21.3 \mathrm{bpm}$ at the end of bedrest $(p<0.0001)$ consistent with cardiovascular deconditioning. Cardiac-output decreased from $6.6 \pm 0.9 \mathrm{l} / \mathrm{min}$ to $6 \pm 1 \mathrm{1} / \mathrm{min}$ at end of bed rest (recovery: $6.8 \pm 1.2 \mathrm{l} / \mathrm{min}, p=0.0096)$. Left-ventricular ejection fraction and mass-index did not change. Echocardiographic global longitudinal strain (baseline: $-19.90 \pm 2.1 \%$; bedrest: $-18.1 \pm 2.1 \%$, $p<0.0001)$ decreased, whereas global circumferential strain in MRI tended to increase (baseline: - $18.6 \pm 1.7 \%$; bedrest: $19.1 \pm 1.6 \%, p=0.0843$ ). After 4 days of recovery MRI measurements had returned to baseline.

Conclusion: In healthy persons, cardiovascular deconditioning through 60 days strict head-down promotes orthostatic tachycardia in the absence of cardiac atrophy or sustained reductions in myocardial performance. The rapid reversal of the phenomenon following reambulation is not commonly seen in patients with the postural tachycardia syndrome.

Funding: This work was supported by NASA, ESA and programmatic funding of the German Aerospace Center (DLR). FH received funding by the German Aerospace Center (DLR) and the German Federal Ministry of Economy and Technology, BMWi, 50WB1816).

\section{Virtual Poster \#33}

\section{Heart rate variability in healthcare workers with and without preschool children}

B. De Maria ${ }^{1}$, V. De Grazia ${ }^{1}$, G. Cassetti ${ }^{1}$, L. Clementi ${ }^{2}$, F. Perego ${ }^{1}$, A. Porta ${ }^{3,4}$, L.A. Dalla Vecchia ${ }^{1}$

${ }^{1}$ IRCCS Istituti Clinici Scientifici Maugeri, Istituto di Milano, Milan, Italy; ${ }^{2}$ Department of Electronics Information and Bioengineering, Politecnico of Milan, Milan, Italy; ${ }^{3}$ Department of Biomedical Sciences for Health, University of Milan, Milan, Italy; ${ }^{4}$ Department of Cardiothoracic, Vascular Anesthesia and Intensive Care, IRCCS Policlinico San Donato, Milan, Italy

The conciliation between career and family is a relevant ongoing issue for working women and men worldwide. The more and more dynamic and demanding working activities outside home on one side, and the family needs on the other side are potential stressors influencing the workers' health. The literature lacks evidence about the state of the cardiac autonomic regulation of young parents with preschool children, whose conciliation of work and family duties may be highly demanding, in particular for workers with responsibility, such as the healthcare professionals. The aim of the study was to compare the cardiac autonomic profile of men and women, working as healthcare professionals, with and without children of preschool age. We enrolled 50 men and 50 women working at our hospital: 25 men (MKIDS) and 25 women (WKIDS) had preschool children, while 25 men (MNOKIDS) and 25 women (WNOKIDS) did not. A standard 24-h electrocardiogram was obtained during a regular working day. Long-term power spectral analysis was performed on the RR interval (RR) series during daytime (DAY) and nighttime (NIGHT). RR variance $\left(\sigma_{\mathrm{RR}}^{2}\right)$ was calculated and the power in high frequency band $\left(\mathrm{HF}_{\mathrm{RR}}\right)$ was considered as an index of the vagal modulation directed to the sinus node. As to the male population, $\sigma_{\mathrm{RR}}^{2}$ and $\mathrm{HF}_{\mathrm{RR}}$ increased during NIGHT compared to DAY in both MNOKIDS (3460 \pm 2068 vs. $4629 \pm 3433 \mathrm{~ms}^{2}$ and $363 \pm 378$ vs. $1181 \pm 1245 \mathrm{~ms}^{2}$, respectively) and MKIDS $\left(2391 \pm 1195\right.$ vs. $3972 \pm 3192 \mathrm{~ms}^{2}$ and $150 \pm 92$ vs. $1221 \pm 1243 \mathrm{~ms}^{2}$, respectively). As to the female population, the expected increase of $\sigma_{\mathrm{RR}}^{2}$ and $\mathrm{HF}_{\mathrm{RR}}$ was observed only in the WNOKIDS $\left(3334 \pm 2153\right.$ vs. $4816 \pm 4063 \mathrm{~ms}^{2}$ and $356 \pm 334$ vs. $1397 \pm 1629 \mathrm{~ms}^{2}$, respectively). Both $\sigma_{\mathrm{RR}}^{2}$ and $\mathrm{HF}_{\mathrm{RR}}$ were higher in WNOKIDS than in WKIDS during NIGHT $\left(4816 \pm 4063\right.$ vs. $2336 \pm 3170 \mathrm{~ms}^{2}$ and $1397 \pm 1629$ vs. $556 \pm 950 \mathrm{~ms}^{2}$, respectively). These results suggest that the presence of preschool children modifies the cardiac neural control of mothers, but not that of male working parents. We hypothesize that the lower nocturnal cardiac vagal modulation that characterizes women in presence of children might represent a physiological and finalistic adaptation to favor a prompt reaction in case of any need of the child.

\section{Virtual Poster \#34}

The effect of analgesic fentanyl administration on sympathetic and cardiovascular responses in humans during simulated hemorrhage

J.C. Watso ${ }^{1}$, M. Huang ${ }^{1,2}$, L.N. Belval ${ }^{1}$, F.A. Cimino III ${ }^{1}$, J.M. Hendrix ${ }^{1,3}$, C. Hinojosa-Laborde ${ }^{4}$, C.G. Crandall ${ }^{1}$

${ }^{1}$ Institute for Exercise and Environmental Medicine, Texas Health Presbyterian Hospital Dallas and University of Texas Southwestern Medical Center, Dallas, TX, USA; ${ }^{2}$ Department of Applied Clinical Research, University of Texas Southwestern Medical Center, Dallas, TX, USA; ${ }^{3}$ Department of Anesthesiology, University of Texas 
Southwestern Medical Center, Dallas, TX, USA; ${ }^{4}$ US Army Institute of Surgical Research, JBSA Fort Sam, Houston, TX, USA

Introduction: Fentanyl is a leading pre-hospital analgesic option following military or civilian trauma. However, it is unknown if fentanyl impairs hemorrhagic tolerance in humans.

Purpose: The purpose of this investigation was to test the hypothesis that fentanyl administration in conscious humans impairs tolerance to simulated hemorrhage via diminished sympathetic and cardiovascular compensation.

Methods: Twenty-two adults (9 females/13 males; age: $28 \pm 7$ years; body mass index: $26 \pm 3 \mathrm{~kg} \mathrm{~m}^{-2}$; BP: systolic $120 \pm 10$, diastolic $75 \pm 10 \mathrm{mmHg}$ ) completed this randomized, crossover, placebocontrolled trial during two visits to the laboratory. One minute after intravenous administration of either fentanyl $(75 \mu \mathrm{g})$ or placebo (saline), tolerance to simulated hemorrhage was assessed via progressive lower-body negative pressure (LBNP). The LBNP protocol started at $40 \mathrm{mmHg}$ and decreased by $10 \mathrm{mmHg}$ (i.e., $40,50 \mathrm{mmHg}$, etc.) every $3 \mathrm{~min}$ until the participant reached pre-syncope. Hemorrhagic tolerance was quantified as a cumulative stress index ( $\mathrm{mmHg} \mathrm{min})$ and compared between trials using paired, two-tailed t-tests. Muscle sympathetic nerve activity (MSNA; radial microneurography, $\mathrm{n}=12$ paired recordings), beat-to-beat BP (finger photoplethysmography), and heart rate (electrocardiogram) were compared between trials using two-way repeated measures ANOVAs with main factors of time point (pre-infusion, post-infusion, LBNP 40, LBNP 50, and the final $20 \mathrm{~s}$ of LBNP) and drug (fentanyl versus placebo).

Results: LBNP tolerance was not different between trials (fentanyl: $570 \pm 345$, placebo: $618 \pm 263 \mathrm{mmHg} \min , \quad p=0.48$ ). During LBNP, MSNA (time: $\mathrm{p}<0.01$, drug: $\mathrm{p}=0.21$, interaction: $\mathrm{p}=0.34$ ), BP (time: $p<0.01$, drug: $p=0.08$, interaction: $p=0.29$ ), and heart rate (time: $p<0.01$, drug: $p=0.79$, interaction: $p=0.03 ; p>0.08$ for all post hoc comparisons) responses were not different between trials.

Conclusions: These preliminary data suggest that administration of an analgesic dose of fentanyl does not reduce tolerance to simulated hemorrhage. These data also suggest that fentanyl administration does not attenuate increases in MSNA or BP responses during progressive central hypovolemia.

Funding: Department of Defense-US Army W81XWH1820012 (CGC).

\section{Virtual Poster \#35}

\section{Muscle metaboreflex control of sympathetic activity is preserved in healthy males following acute intermittent hypercapnic hypoxia}

B.M. Shafer ${ }^{1}$, T.D. Vermeulen ${ }^{1}$, A.V. Incognito ${ }^{2}$, M. Nardone ${ }^{2}$, A.L. Teixeira' ${ }^{2}$, J. Benbaruj ${ }^{1}$, P.J. Millar ${ }^{2}$, G.E. Foster ${ }^{1}$

${ }^{1}$ Centre for Heart, Lung and Vascular Health, School for Health and Exercise Science, University of British Columbia, Kelowna, Canada; ${ }^{2}$ Department of Human Health and Nutritional Sciences, University of Guelph, Guelph, Ontario, Canada

Introduction: Obstructive sleep apnea (OSA) patients experience intermittent hypercapnic hypoxia (IHH) during sleep, resulting in elevated sympathetic activity during wakefulness. However, the change in muscle sympathetic nerve activity (MSNA) during postexercise circulatory occlusion (PECO) is reduced in OSA, suggesting impaired skeletal muscle metaboreflex control. IHH also yields longlasting sympathoexcitation in healthy humans, but its impact on the metaboreflex is unknown. We hypothesized that IHH would increase
MSNA and attenuate the change in MSNA during muscle metaboreflex activation (MMA) in healthy men.

Methods: Thirteen men (age $=24 \pm 4$ years; $B M I=24 \pm 4 \mathrm{~kg} / \mathrm{m}^{2}$ ) were exposed to IHH consisting of 40 cycles of $40 \mathrm{~s}$ of hypercapnic hypoxia (end-tidal $\mathrm{PCO}_{2}: \quad+4 \pm 0 \mathrm{mmHg}$; end-tidal $\mathrm{PO}_{2}$ : $60 \pm 0 \mathrm{mmHg}$ ) and $20 \mathrm{~s}$ of normoxia. Blood pressure (BP; photoplethysmography), heart rate (HR; lead II ECG), and MSNA (microneurography) were acquired at baseline, during 2-min of submaximal isometric handgrip exercise, and 4-min of PECO before and after IHH. MSNA was quantified as burst frequency (BF) and total activity (TA). Pressor and MSNA response to handgrip exercise and MMA before and after IHH were compared using mixed effects linear models. Data are means \pm standard deviation or $95 \%$ confidence intervals $(\mathrm{CI})$.

Results: IHH increased MSNA BF (15 \pm 7 vs. $20 \pm 6$, burst/min; $\mathrm{P}<0.01), \quad$ TA $\quad(686 \pm 290$ vs. $1115 \pm 371$, arbitrary units; $\mathrm{P}<0.001)$ and diastolic $\mathrm{BP}(66 \pm 7$ vs. $70 \pm 7, \mathrm{mmHg} ; \mathrm{P}=0.05)$ at rest. In response to handgrip exercise, the changes in BP, HR, MSNA $\mathrm{BF}$, and TA were similar pre- and post-IHH (All $\mathrm{P}>0.36$ ). During PECO, the change in MSNA BF and TA were similar following IHH; however, the change in diastolic BP $(\Delta 10\{95 \% \mathrm{CI} 6,13\}$ vs. $\Delta 11\{7,15\}, \mathrm{mmHg} ; \mathrm{P}<0.05)$ and systolic BP $(\Delta 16\{95 \% \mathrm{CI} 10,22\}$ vs. $\Delta 18\{12,23\}, \mathrm{mmHg} ; \mathrm{P}<0.05)$ from baseline were modestly increased following IHH.

Conclusions: IHH leads to sympathoexcitation and increased DBP without affecting the pressor response to exercise. Contrary to our hypothesis, the sympathetic response to MMA during PECO was preserved even though the pressor response was slightly augmented. These data would suggest that in healthy men, acute IHH does not alter muscle metaboreflex control of sympathetic activity.

Funding: Natural Sciences and Engineering Research Council of Canada, Heart and Stroke Foundation of Canada, and the Michael Smith Foundation for Health Research.

\section{Virtual Poster \#36}

In-vivo recordings from the human vagus nerve using ultrasoundguided microneurography

M.M. Ottaviani ${ }^{1}$, L. Wright ${ }^{2}$, T. Dawood ${ }^{2}$, V.G. Macefield ${ }^{2,3}$

${ }^{1}$ Scuolo Superiore Sant'Anna, Pisa, Italy; ${ }^{2}$ Baker Heart and Diabetes Institute, Melbourne, Australia; ${ }^{3}$ Department of Physiology, University of Melbourne, Melbourne, Australia

Intraneural microelectrodes have been used extensively to record from single somatosensory axons supplying muscle, tendons, joints and skin, and to record from postganglionic sympathetic axons supplying muscle and skin, in accessible peripheral nerves in awake humans. However, never has the vagus nerve been targeted, no doubt because of its close proximity to the carotid artery and jugular vein in the neck. Here we report the first unitary recordings from the human cervical vagus nerve, obtained using ultrasound-guided insertion of tungsten microelectrodes into fascicles of the nerve. We identified tonically-active neurones in which firing rates were inversely related to heart rate (and directly related to cardiac interval), which we classified as putative preganglionic parasympathetic axons directed to sinoatrial node of the heart. We also recorded from tonically-active presumed sensory axons from the airways and presumed motor axons to the larynx. This new methodology opens exciting new opportunities to study the physiology of the human vagus nerve in health and disease.

Funding: Baker Heart and Diabetes Institute. 


\section{Virtual Poster \#37}

\section{Evidence of sympathetic regulation of the choroid circulation} by baroreceptors in humans

S.W. Holwerda $a^{1,2,6}$, R.H. Kardon ${ }^{3,4,5}$, R. Hashimoto ${ }^{5}$ J.K. Nellis ${ }^{5}$ J.M. Ful1 ${ }^{5}$, G.L. Pierce 1,2

${ }^{1}$ Department of Health and Human Physiology, University of Iowa, Iowa City, IA, USA; ${ }^{2}$ Abboud Cardiovascular Research Center, University of Iowa, Iowa City, IA, USA; ${ }^{3}$ Iowa City VA Center for Prevention and Treatment of Visual Loss, Iowa City, IA, USA; ${ }^{4}$ Department of Veteran Affairs Hospital Iowa City, Iowa City, IA, USA; ${ }^{5}$ Department of Ophthalmology and Visual Sciences, Iowa City, IA, USA; ${ }^{6}$ Department of Anesthesiology, University of Kansas Medical Center, Kansas City, KS, USA

Background: The choroid is the most vascularized structure of the eye with dense sympathetic innervation. Impairment in neural control of choroidal blood flow occurs with ocular diseases, such as glaucoma and age-related macular degeneration. Therefore, we sought to determine whether sympathetic regulation of choroid blood flow is influenced by baroreceptor input.

Hypothesis: We hypothesized that choroid vascular resistance would be significantly elevated during reflex increases in sympathetic activity during baroreceptor unloading compared with retinal arterioles that do not receive sympathetic innervation. Further, we hypothesized that that increases in sympathetic nerve activity and choroid vascular resistance during cardiopulmonary baroreceptor unloading would be positively correlated.

Methods: 10 healthy participants (6 women/4 men) of varying age (22-73 years) without history of ophthalmic disease and/or surgery underwent measurements of ocular blood flow (Laser Speckle Flowgraphy), ocular perfusion pressure, and muscle sympathetic nerve activity (MSNA, microneurography), before and during baroreceptor unloading via mild steady-state lower body negative pressure (LBNP). Responses to LBNP at -15 and $-30 \mathrm{mmHg}$ were averaged for each participant to account for inherent differences in sensitivity to the stimulus.

Results: As expected, significant increases in MSNA were observed during LBNP compared with baseline (43 \pm 7 vs. $29 \pm 9$ bursts $100 \mathrm{hb}^{-1}, \mathrm{P}=0.002, \mathrm{n}=8$ ). In response to LBNP, choroid vascular resistance in the high-flow region was significantly increased compared with baseline $\left(3.5 \pm 0.5\right.$ vs. $3.1 \pm 0.5 \mathrm{mmHg} \bullet \mathrm{AU}^{-1}$, $\mathrm{P}=0.008)$. Interestingly, vascular resistance in the choroid low-flow region (watershed zone) tended to be reduced during LBNP $\left(9.8 \pm 1.4\right.$ vs. $\left.11.4 \pm 1.4 \mathrm{mmHg} \bullet \mathrm{AU}^{-1}, \quad \mathrm{P}=0.052\right)$, suggesting reduced sympathetic-mediated vasoconstriction. Moreover, no change in resistance was observed in retinal arterioles lacking sympathetic innervation $(\mathrm{P}=0.84)$. Importantly, percent increases in choroid vascular resistance were positively correlated with observed increases in MSNA ( $\mathrm{R}=0.76, \mathrm{P}=0.028)$. No relation between vascular resistance and MSNA was observed in the choroid watershed zone $(\mathrm{R}=-0.01, \mathrm{P}=0.99)$.

Conclusion: These preliminary findings suggest that sympathetic vasoconstriction in the choroid occurs during baroreceptor unloading in humans, particularly in the high-flow region of the choroid (nonwatershed zone). Further studies involving sympathetic blockade at the choroid during baroreceptor unloading are needed to confirm and extend these findings.

Funding: This research was supported by AHA 17POST33440101 and T32 HL07121 NHLBI (S.W.H.), and AHA 13SDG143400012 (G.L.P.) and U54 TR001356 (University of Iowa) and the Iowa City VA Center for the Prevention and Treatment of Visual Loss C9251-C (RX003002).

\section{Virtual Poster \#38}

Muscle sympathetic single-unit response patterns during graded muscle metaboreflex activation in young healthy adults

A.V. Incognito ${ }^{1}$, M. Nardone ${ }^{1}$, A.L. Teixeira ${ }^{1}$, J.B. Lee ${ }^{1}$, M.M. Kathia ${ }^{1}$, P.J. Millar ${ }^{1,2}$

${ }^{1}$ Department of Human Health and Nutritional Sciences, University of Guelph, Guelph, Ontario, Canada; ${ }^{2}$ Toronto General Research Institute, Toronto, Ontario, Canada

Background: In response to a sympathoexcitatory stimulus in humans, most muscle sympathetic single units within a nerve bundle demonstrate increases in firing frequency (i.e., are activated). However, we have observed small single-unit subpopulations that can also be simultaneously inhibited (decrease firing frequency) in response to the same stimulus. The control systems underpinning this level of differential control are largely unknown. We investigated whether muscle sympathetic single units can behave bidirectionally in response to a single stimulus, dependent on the level of the sympathoexcitation.

Methods: Fifty-three muscle sympathetic single units (microneurography) were recorded in 17 participants ( 8 women; $24 \pm 3$ years). Five $40 \mathrm{~s}$ bouts of $10 \%$ static handgrip were performed during a $10 \mathrm{~min}$ forearm ischemia to progressively increase metabolite accumulation. Each static handgrip was separated by a $75 \mathrm{~s}$ ischemic rest (post-exercise circulatory occlusion [PECO]) to assess the isolated action of the muscle metaboreflex. During each set of PECO, individual single units were classified as activated, non-responsive, or inhibited if the spike frequency was above, within, or below the baseline variability, respectively.

Results: From sets $1-5$ of PECO, the proportion of single units with activated $(34,45,68,87,89 \%)$, non-responsive $(43,44,23,7,9 \%)$, or inhibited $(23,11,9,6,2 \%)$ responses changed $(\mathrm{P}<0.001)$ as total muscle sympathoexcitation increased (baseline vs. set 1-5: $684 \pm 280$ vs. $762 \pm 340 \quad[P>0.99], \quad 849 \pm 355 \quad[P=0.07]$ $1079 \pm 355 \quad[P<0.001], \quad 1313 \pm 311[P<0.001], \quad 1639 \pm 398$ $[P<0.001]$ arbitrary units/min; all $P$. Overall, $51 / 53(96 \%)$ single units were activated in at least one set of PECO, with $16(31 \%)$ of these first demonstrating an initially inhibited response prior to activation. Those single units with an initially inhibited response pattern had delayed activation onset compared to non-inhibited single units (set $3 \pm 1$ vs. $2 \pm 1, \mathrm{P}<0.001$ ). Once activated, the spike frequency rate-of-rise was similar between single-unit subgroups $(8.5 \pm 6.5$ vs. $7.1 \pm 6.0$ spikes $/ \mathrm{min} / \mathrm{set}, \mathrm{P}=0.48$ ).

Conclusions: Muscle sympathetic single-unit firing demonstrated differential control during muscle metaboreflex activation. Single units that were initially inhibited during graded metaboreflex activation were capable of being activated in later sets. These findings reveal that single-unit activity is influenced by convergent inhibitory and excitatory neural inputs which yield heterogenous single-unit activation thresholds.

Funding: A.V.I.-Natural Science and Engineering Research Council of Canada (NSERC) Canada Graduate Scholarship; M.N.-Ontario Graduate Scholarship; J.B.L.-Natural Science and Engineering Research Council of Canada (NSERC) Graduate Scholarship; P.J.M.-NSERC Discovery Grant (No. 06019), Canada Foundation for Innovation (No. 34379), Ontario Ministry of Research, Innovation, and Science (No. 34379), Ontario Early Researcher Award (1814-288). 


\section{Virtual Poster \#39}

Six months of exercise-based cardiac rehabilitation enhances sympathetic neural recruitment strategies during maximal endinspiratory apnea

A.W. D'Souza ${ }^{1}$, M.B. Badrov ${ }^{1}$, S. Lalande ${ }^{2}$, N. Suskin ${ }^{3,4}$, J.K. Shoemaker ${ }^{1,5}$

${ }^{1}$ School of Kinesiology, Western University, London, Ontario, Canada; ${ }^{2}$ Department of Kinesiology and Health Education, The University of Texas at Austin, Austin, TX, USA; ${ }^{3}$ Cardiac Rehabilitation and Secondary Prevention Program of St. Joseph's Health Care London, London, Ontario, Canada; ${ }^{4}$ Department of Medicine (Cardiology) and Program of Experimental Medicine, Western University, London, Ontario, Canada; ${ }^{5}$ Department of Physiology and Pharmacology, Western University, London, Ontario, Canada

Exercise-based cardiac rehabilitation (CR) improves autonomic function in ischemic heart disease (IHD) patients. However, the effect of $\mathrm{CR}$ on sympathetic neural recruitment remains unknown. We therefore evaluated the hypothesis that six months of CR would enhance sympathetic neural recruitment in response to a maximal end-inspiratory apnea (EI-apnea) in IHD patients with preserved ejection fraction. Eight IHD patients ( 1 female; $62 \pm 9$ years, $173 \pm 11 \mathrm{~cm}, 84 \pm 9 \mathrm{~kg}$ ) were studied prior-to and following six months of aerobic and resistance training-based CR. We measured heart rate (HR; electrocardiogram), blood pressure (finger plethysmography) and muscle sympathetic nerve activity (MSNA; microneurography) during an EI-apnea, pre- and post-CR. Sympathetic action potentials (AP) were detected from the filtered raw MSNA signal using a wavelet-based algorithm which quantifies the number and morphology of APs within a recording segment, enabling detection of changes in firing patterns and AP recruitment from baseline to periods of stress. Data were analyzed during a 1-min baseline and the second half $(\sim 30 \mathrm{~s})$ of the EI-apnea. Values are mean \pm standard deviation. EI-apnea duration was similar pre- and post-CR ( $28 \pm 7$ vs. $35 \pm 19 \mathrm{~s}, P=0.25$ ). Mean arterial pressure during the EI-apnea was $91 \pm 15 \mathrm{mmHg}$ pre-CR and $85 \pm 14 \mathrm{mmHg}$ post-CR $(P=0.07)$, while HR remained unaltered $(66 \pm 12$ vs. $63 \pm 11 \mathrm{bpm}$, pre-CR vs. post-CR, respectively, $P=0.41$ ). CR reduced baseline MSNA burst frequency ( $46 \pm 9$ to $32 \pm 11$ bursts/min, $P<0.01$ ), whereas EI-apnea burst frequency trended toward a reduction post-CR $(59 \pm 13$ to $51 \pm 11$ bursts/min, $P=0.10)$. Thus, the change in MSNA burst frequency $(\Delta 12 \pm 9 \mathrm{vs.}$ $\Delta 18 \pm 14$ bursts/min, $P=0.23)$ and burst amplitude $(\Delta 15 \pm 11$ vs. $\Delta 23 \pm 16$ AU, $P=0.20$ ) from baseline to EI-apnea were not altered by CR. Nonetheless, CR elicited a greater change from baseline to EIApnea in AP frequency $(\Delta 233 \pm 229$ to $\Delta 373 \pm 318$ spikes $/ \mathrm{min}$, preCR to post-CR, respectively, $P=0.04$ ), AP clusters per burst $(\Delta 0.3 \pm 0.6$ to $\Delta 0.8 \pm 0.4$ clusters/burst, $P=0.03)$, cluster recruitment $(\Delta 0 \pm 2$ to $\Delta 2 \pm 2$ clusters, $P=0.01)$, and APs per burst ( $\Delta 2 \pm 2$ to $\Delta 4 \pm 3$ spikes/burst, $P=0.04$ ). These data indicate that 6 months of CR increased sympathetic neural recruitment in response to an EI-apnea via increased firing of previously recruited axons and recruitment of latent subpopulations of higher-threshold axons.

Funding: Canadian Institutes of Health Research (Grant no. 217532; awarded to J.K. Shoemaker).

\section{Virtual Poster \#40}

Effect of sensory blockade and rate of sensory stimulation on local heating induced axon reflex response in facial skin

K. Metzler-Wilson ${ }^{1,2}$, T.E. Wilson ${ }^{3}$, S.M. Ausmus ${ }^{1}$, A. Sventeckis ${ }^{1}$

${ }^{1}$ Department of Physical Therapy, School of Health \& Human

Sciences, School of Medicine, Indiana University, Indianapolis, IN, USA; ${ }^{2}$ Departments of Dermatology and Anatomy, Cell Biology, \& Physiology, School of Medicine, Indiana University, Indianapolis, IN, USA; ${ }^{3}$ Division of Biomedical Sciences, College of Osteopathic Medicine, Marian University, Indianapolis, IN, USA

Local neuronal circuits in non-glabrous skin drive the initial increase of the biphasic cutaneous vasodilation response to rapid non-noxious heating. This axon reflex response is abolished with non-glabrous forearm cutaneous sensory blockade via voltage-sensitive $\mathrm{Na}^{+}(\mathrm{NaV})$ channel inhibition, but investigations in skin associated with flushing pathology are sparse. Slow local heating does not engage forearm skin axon reflexes, but this neuronal activation threshold has not been investigated in facial skin. We hypothesized that despite regional differences in sensory afferents, both sensory blockade and slowing the rate of heating would abate the cutaneous axon reflex-mediated vasodilator responses. Skin blood flow (laser-Doppler flowmetry) responses of 6 healthy subjects ( 5 female) to non-noxious local heating in the forearm, cheek, and forehead were measured and expressed as a percentage of maximum cutaneous vascular conductance $(\mathrm{CVC}=$ flux/mean arterial pressure $) . \mathrm{CVC}$ was assessed during rapid $\left(1{ }^{\circ} \mathrm{C} / 30 \mathrm{~s}\right)$ and slow $\left(1{ }^{\circ} \mathrm{C} / 10 \mathrm{~min}\right)$ local heating to $43{ }^{\circ} \mathrm{C}$ in both $\mathrm{NaV}$ inhibition (topical $2.5 \%$ lidocaine/prilocaine) and control conditions. Sensory blockade decreased forearm (control: $84 \pm 4$, block: $34 \pm 9 \%, \mathrm{p}<0.001)$ and forehead (control: $90 \pm 3$, block: $68 \pm 3 \%, \mathrm{p}=0.022$ ) initial peaks but did not alter cheek responses (control: $90 \pm 3$, block: $92 \pm 13 \%$ ) to rapid heating. Slow heating decreased the initial peak for all sites (forearm: $16 \pm 2$, forehead: $36 \pm 3$, cheek: $34 \pm 5 \%$, all $p<0.001)$. Control experiments combining slow heating and $\mathrm{NaV}$ inhibition did not further reduce responses. Slower rate of sensory afferent activation eliminates the axon reflex response in both facial and non-glabrous skin, but sensory blockade was ineffective in blocking axon reflex responses in flushing-prone cheek skin.

Funding: This work was supported by the National Institutes of Health [AR069912]. Additional support was provided by Marian University College of Osteopathic Medicine and the Federal Work Study program.

\section{Virtual Poster \#41}

Cardiorespiratory responses to upper and lower body isometric exercise or passive movement

\section{T.J. Pereira, H. Edgell \\ York University, Kinesiology and Health Sciences, Toronto, ON, Canada}

Muscle size should be an important consideration for metaboreflex and mechanoreflex studies, yet the isolation of these reflexes often involves only one muscle group. Recent work from our lab has indicated that limb-specific differences could exist. In 10 men (age $23 \pm 2$; BMI $27 \pm 5 \mathrm{~kg} / \mathrm{m}^{2}$ ) and 5 women (age $21 \pm 2$; BMI $21 \pm 1 \mathrm{~kg} / \mathrm{m}^{2}$ ), we investigated the pressor and ventilatory responses to arm or leg metaboreflex and mechanoreflex activation. Trials were: (1) 2 min of isometric arm (40\% MVC) or leg ( $80 \%$ MVC) exercise followed by $3 \mathrm{~min}$ of post-exercise circulatory occlusion (PECO), and 
(2) 3 min of arm or leg passive movement (PM). Ventilation (VE) and mean arterial pressure (MAP) were continuously measured (Nexfin, pneumotach). As expected, VE and MAP increased during arm and leg exercise (all $\mathrm{P}<0.006$ ). During leg PECO, MAP remained above baseline ( $89 \pm 2$ vs. $85 \pm 2 \mathrm{mmHg}, \mathrm{P}=0.007)$, while VE returned to baseline $(\mathrm{P}=1.00)$. Similarly, during arm PECO, MAP remained above baseline $(97 \pm 3$ vs. $83 \pm 3 \mathrm{mmHg}, \mathrm{P}<0.001)$, while $\mathrm{VE}$ returned to baseline levels $(\mathrm{P}=0.344)$. VE was similar between arm and leg PECO $(\mathrm{P}=0.357)$, yet MAP was higher during arm PECO ( $97 \pm 3$ vs. $89 \pm 2 \mathrm{mmHg}, \mathrm{P}=0.006$ ). During the first $30 \mathrm{~s}$ of leg $\mathrm{PM}, \mathrm{VE}$ increased above baseline $(12.8 \pm 0.5$ vs. $11.3 \pm 0.4 \mathrm{~L} / \mathrm{min}$, $\mathrm{P}=0.001)$ and remained elevated during the final $30 \mathrm{~s}$ of $\mathrm{PM}$ $(13.0 \pm 0.5 \mathrm{~L} / \mathrm{min}, \mathrm{P}=0.004)$. MAP did not change throughout leg PM (all P > 0.241). Arm PM did not change VE from baseline in the first $30 \mathrm{~s} \quad(\mathrm{P}=0.196)$, but increased $\mathrm{VE}$ during the last $30 \mathrm{~s}$ $(12.3 \pm 0.5$ vs. $11.5 \pm 0.5 \mathrm{~L} / \mathrm{min}, \mathrm{P}=0.026)$. Similarly, MAP was not different from baseline during the first $30 \mathrm{~s}$ of arm PM $(\mathrm{P}=0.12)$ but was increased during the last $30 \mathrm{~s}(87 \pm 2$ vs. $83 \pm 2 \mathrm{mmHg}$, $\mathrm{P}=0.004)$. At each timepoint, the $\mathrm{VE}$ and MAP responses to arm or leg PM were similar (all $\mathrm{P}>0.180$ ). We found (1) greater MAP responses during arm PECO compared to leg PECO, (2) VE increased immediately upon leg PM, yet there was a delay during arm PM, and (3) MAP increased by 3 min of arm PM but not leg PM. Our study suggests that while muscle size may be an important consideration for metaboreflex and mechanoreflex investigations, limb dependent responses may also be present.

Funding: Natural Science and Engineering Council of Canada \#201605289 .

\section{Virtual Poster \#42}

\section{Influence of sex on neural coding strategies during isometric handgrip in young adults}

\author{
L.N. Shoemaker ${ }^{1}$, A.T. Robinson ${ }^{2}$, J.C. Watso ${ }^{3}$, J.L. Greaney ${ }^{4}$, \\ M.M. Wenner ${ }^{1}$ \\ ${ }^{1}$ Department of Kinesiology and Applied Physiology, University of \\ Delaware, Newark, DE, USA; ${ }^{2}$ School of Kinesiology, Auburn \\ University, Auburn, AL, USA; ${ }^{3}$ Institute for Exercise and \\ Environmental Medicine, Texas Health Presbyterian Hospital Dallas, \\ Dallas, TX, USA; ${ }^{4}$ College of Nursing and Health Innovation, \\ University of Texas at Arlington, TX, USA
}

Introduction: Neural control of blood pressure differs between young women and men. Young women generally exhibit lower integrated sympathetic nerve activity at rest, as well as a blunted sympathetic response to isometric handgrip (IHG) exercise compared to men. However, potential sex differences in the underlying sympathetic discharge patterns have not been examined.

Purpose: The purpose of this study was to begin to elucidate sexspecific differences in the magnitude and patterning of sympathetic neural discharge at rest and during IHG.

Methods: Efferent muscle sympathetic nerve activity (microneurography) was measured at baseline and during the final minute of both $30 \%$ IHG and post-exercise ischemia (PEI) in six healthy women $\left(21 \pm 2 \mathrm{y}, 21 \pm 4 \mathrm{~kg} / \mathrm{m}^{2}, 81 \pm 7 \mathrm{mmHg}\right)$ and six healthy men $\left(29 \pm 8 \mathrm{y}, 26 \pm 1 \mathrm{~kg} / \mathrm{m}^{2}\right.$, mean blood pressure: $90 \pm 10 \mathrm{mmHg}$ ). Sympathetic action potential (AP) discharge patterns were examined using wavelet-based methodology.

Results: Integrated burst frequency (women $11 \pm 3$ vs. men $10 \pm 3$ bursts/min; $\mathrm{p}=0.76$ ) and AP firing (women $131 \pm 116$ vs. men $59 \pm 27 \mathrm{APs} / \mathrm{min}, \mathrm{p}=0.19$ ) at rest were not different between groups. The increases in integrated burst frequency during IHG and PEI (both $\mathrm{p}<0.01$ vs. baseline) were not different between groups (interaction: $\mathrm{p}=0.56$ ). Similarly, increases in AP firing during IHG (women $\Delta 125 \pm 131$ vs. men $\Delta 97 \pm 108 \mathrm{APs} / \mathrm{min}$ ) and PEI (women $\Delta 82 \pm 64$ vs. men $\Delta 130 \pm 154$ APs/min; both $\mathrm{p}<0.02$ vs baseline) were not different between men and women (interaction: $\mathrm{p}=0.62$ ). The mean AP content per burst was not different during IHG or PEI (main effect: $p=0.77$ ) or between groups (main effect: $p=0.52$ ). Lastly, the increase in total AP clusters (binned according to peak-topeak amplitude) during IHG (women $\Delta 2 \pm 1$ vs. men $\Delta 4 \pm 2$ ) and PEI (women $\Delta 2 \pm 2$ vs. men $\Delta 3 \pm 3$; both $\mathrm{p} \leq 0.01$ vs. baseline) was not different between groups (interaction: $\mathrm{p}=0.59$ ).

Conclusion: These preliminary data suggest that neural coding strategies to increase sympathetic outflow during IHG and PEI are not different between young men and women; further investigation of the mechanisms supporting sex differences in neural control during sympathoexcitation is needed.

Funding: Supported by AHA 16SDG30700015 and NIH Grants P20 GM113125, 1R01HL146558-01, and HL133414.

\section{Virtual Poster \#43}

\section{Baroreflex sensitivity in premenopausal and postmenopausal women}

S.J. McGinty ${ }^{1}$, E.L. Matthews ${ }^{2}$, J.L. Greaney ${ }^{3}$, M.M. Wenner ${ }^{1}$ ${ }^{1}$ Department of Kinesiology and Applied Physiology, University of Delaware, Newark, DE, USA; ${ }^{2}$ Department of Exercise Science and Physical Education, Montclair State University, Montclair, NJ, USA; ${ }^{3}$ Department of Kinesiology, The University of Texas at Arlington, Arlington, TX, USA

Introduction: Postmenopausal women (POST) have increased muscle sympathetic nerve activity (MSNA) at rest compared to premenopausal women (PRE), an effect that is driven not only by chronological aging but also by the loss of sex hormones. Despite these established increases in sympathetic outflow at rest, whether age-related alterations in sympathetic baroreflex sensitivity (sBRS) are evident in POST remains equivocal.

Purpose: We tested the hypothesis that sBRS would be blunted in POST compared to PRE.

Methods: We examined R-R interval (electrocardiography), beat-bybeat BP (finger photoplethysmography) and MSNA (peroneal microneurography) in 19 PRE (22 \pm 2 years, $\left.22 \pm 3 \mathrm{~kg} / \mathrm{m}^{2}\right)$ and 12 POST ( $57 \pm 5$ year, $\left.24 \pm 2 \mathrm{~kg} / \mathrm{m}^{2}\right)$ during $10 \mathrm{~min}$ of supine rest. Spontaneous sBRS was quantified as the slope of the linear relation between MSNA and diastolic BP. Spontaneous cardiovagal baroreflex sensitivity (cvBRS) was quantified as the slope of the linear relation between $\mathrm{R}-\mathrm{R}$ interval and systolic BP, during sequences in which systolic BP and R-R interval changed in the same direction for four or more consecutive cardiac cycles (HemoLab). Data are presented as mean $\pm \mathrm{SD}$.

Results: Resting systolic BP (PRE $110 \pm 10$ vs. POST $114 \pm 10 \mathrm{mmHg}, P=0.18$ ) and diastolic BP (PRE $69 \pm 8$ vs. POST $70 \pm 8 \mathrm{mmHg}, P=0.72$ ) were not different between PRE and POST, but MSNA at rest was increased in POST (PRE $10 \pm 6$ vs. POST $45 \pm 16$ bursts/100 heart beats, $P<0.0001)$. Spontaneous sBRS was greater in POST (PRE $-1.9 \pm 1.1$ vs. POST $-4.6 \pm 2.3$ bursts/ beat $/ \mathrm{mmHg}, P<0.0005)$, whereas spontaneous cvBRS was reduced (PRE: $29 \pm 13$ vs. POST: $19 \pm 7 \mathrm{~ms} / \mathrm{mmHg}, P<0.05$ ).

Conclusions: Consistent with the existing literature, these data demonstrate blunted cvBRS in POST. However, contrary to our hypothesis, these preliminary data suggest that spontaneous sBRS may be increased in POST. In healthy, normotensive POST, enhanced sensitivity of arterial baroreflex control of MSNA may potentially indicate a preserved ability to buffer fluctuations in BP. 
Funding: Supported by NIH Grants P20 GM113125, P20 GM103446, U54 GM104941, K99/R00 HL133414, and the University of Delaware Research Foundation.

\section{Virtual Poster \#44}

\section{Sympathetic neural response to acute thermal sensations}

H.A. Cunningham ${ }^{1}$, J.R. Carter $^{2}$

${ }^{1}$ Department of Kinesiology, Michigan Technological University, Houghton, MI, USA; ${ }^{2}$ Department of Health \& Human Development, Montana State University, Bozeman, MT, USA

The influence of thermal stimuli on the sympathetic nervous system is variable and largely depends on the change in temperature and timing of the stimuli. Core temperature changes yield increased muscle sympathetic nerve activity (MSNA) while changes in skin temperature yield variable MSNA responses. The MSNA responses to acute heating or cooling sensations remains unclear. Twenty-three participants (11 women, $12 \mathrm{men}$; age $24 \pm 1$ years, BMI $26 \pm 1 \mathrm{~kg} / \mathrm{m}^{2}$ ) underwent a thermal protocol that included four trials each of cool sensation threshold, warm sensation threshold, and heat pain (12 total trials). Continuous blood pressure (finger plethysmography), heart rate (electrocardiography), and muscle sympathetic nerve activity (microneurography) were recorded throughout all trials. A $\log$ transformation was utilized for non-normal data, and assessed with a Shapiro-Wilk test. For data with normal distribution, t-tests were used to compare physiological data for cool and warm sensation, and repeated measures ANOVA to compare multiple heat pain data points. Mean arterial pressure (MAP) and MSNA were significantly attenuated during the immediate recovery of cool sensation (MAP, $\Delta-1.1 \pm 0.3 \mathrm{mmHg}, \mathrm{p}=0.001 ;$ MSNA,$\Delta-2.3 \pm 0.6 \mathrm{bursts} / \mathrm{min}$, $\mathrm{p}=0.001)$. Similarly, MSNA was inhibited during the immediate recovery of warm sensation $(\Delta-3.8 \pm 1.1$ bursts $/ \mathrm{min}, \mathrm{p}=0.003)$. Finally, MSNA was inhibited during the sensation of heat pain $(\Delta-3.5 \pm 1.0$ bursts/min; $\mathrm{p}=0.002)$ and MAP was reduced during the recovery from heat pain $(\Delta 0.7 \pm 0.6 \mathrm{mmHg}, \mathrm{p}=0.021)$. Heart rate was attenuated during sensation compared to recovery (sensation, $\Delta-1.3 \pm 0.7 \mathrm{bpm}$; recovery, $\Delta 1.6 \pm 0.5 \mathrm{bpm} ; \mathrm{p}<0.001)$. There were no significant differences between men and women for any variables and responses. These results indicate that acute thermal sensations result in the inhibition of MSNA.

Funding: Songer Research Award for Human Health Research.

\section{Virtual Poster \#45}

\section{Divergent autonomic responses to tonic pain: what happens to baroreflex control of blood pressure?}

C.E. Taylor ${ }^{1,2}$, S. Kobuch ${ }^{2}$, J. Wu ${ }^{2}$, A. Burton ${ }^{2,3}$, V.G. Macefield ${ }^{3,4}$ ${ }^{1}$ School of Health Sciences, Western Sydney University, Sydney, Australia; ${ }^{2}$ School of Medicine, Western Sydney University, Sydney, Australia; ${ }^{3}$ Neuroscience Research Australia, Sydney, Australia; ${ }^{4}$ Baker Heart and Diabetes Institute, Melbourne, Australia

Chronic pain may have serious physiological consequences that affect the cardiovascular system. Our group has previously identified divergent responses of muscle sympathetic nerve activity (MSNA) in response to an experimental model of tonic pain. However, it is not clear what effect this may have on beat-to-beat control of blood pressure. We hypothesise that individuals who experience an increase in MSNA during pain (positive responders) demonstrate a reduction in sympathetic baroreflex sensitivity (BRS) and an upward resetting of the baroreflex to higher pressures and sympathetic outflow. We performed retrospective analysis on data from 30 participants (15 male, 15 female) in whom MSNA (microneurography), blood pressure and ECG measurements were made continuously during rest and a tonic pain protocol. Pain was induced using an infusion of hypertonic saline into the tibialis anterior muscle in order to produce a dull, muscular ache. 5-min periods during rest and peak pain were extracted and sympathetic BRS was quantified as the relationship between diastolic pressure and MSNA burst incidence. To assess baroreflex resetting we calculated the T50 value (diastolic pressure at which $50 \%$ of cardiac cycles are associated with a burst of MSNA) and the error signal (difference between average diastolic pressure and T50). Whilst both groups experienced elevated blood pressure during pain, we report no significant change in sympathetic BRS from rest to peak pain in positive responders ( -1.9 vs. -1.7 bursts/100 $\mathrm{hb} / \mathrm{mmHg}$ ) and negative responders ( -1.9 vs. -1.8 bursts/100 hb/ $\mathrm{mmHg}, \mathrm{P}>0.05)$. In positive responders there was an upward resetting of the baroreflex slope by an average of $25 \mathrm{mmHg}$ and a shift in the operating point, meaning that individuals were operating at higher pressures and higher levels of MSNA. In negative responders, there was a downward resetting of the baroreflex by an average of $16 \mathrm{mmHg}$ and a change in the error signal so that individuals were operating at slightly higher pressures but lower levels of MSNA. The latter response may reflect a 'healthier' adjustment of the baroreflex during tonic pain, whereas the upward resetting of the baroreflex in positive responders could manifest in chronically elevated sympathetic outflow and poor blood pressure outcomes over time.

Funding: This work was supported by the National Health \& Medical Research Council of Australia (GNT1029782 and GNT11000420).

\section{Virtual Poster \#46}

Exploring interactions between apneic and baroreflexmechanisms governing sympathetic neural recruitment strategies in human muscle sympathetic nerve activity

B.S. Kulas ${ }^{1}$, S.A. Klassen ${ }^{1,3}$, M.E. Moir ${ }^{1}$, J.K. Shoemaker ${ }^{1,2}$

${ }^{1}$ Neurovascular Research Laboratory, School of Kinesiology,

${ }^{2}$ Department of Physiology and Pharmacology, University of Western Ontario, London, Ontario, Canada; ${ }^{3}$ Department of Anesthesiology and Perioperative Medicine, Mayo Clinic, Rochester, MN, USA

The sympathetic nervous system employs recruitment strategies to modify human muscle sympathetic nerve activity (MSNA), but the interactions between neural mechanisms governing these strategies are not known. This study investigated the interactive effects of apneic and baroreflex mechanisms on sympathetic neural recruitment strategies. Seven healthy young individuals ( 2 females) were studied using the microneurographic technique (fibular nerve) to quantify action potential (AP) discharge, recruitment, and latency within the (MSNA) filtered neurogram during lower body negative pressure (LBNP) of -10 and $-40 \mathrm{mmHg}$, and during end expiratory apnea (APN) performed at baseline (BSL) and during each level of LBNP. AP discharge increased from BSL $(176 \pm 67 \mathrm{AP} / \mathrm{min}, 8 \pm 2 \mathrm{AP} /$ burst) to $-10 \mathrm{mmHg} \operatorname{LBNP}(241 \pm 63 \mathrm{AP} / \mathrm{min}, 9 \pm 2 \mathrm{AP} / \mathrm{burst})$ and - $40 \mathrm{mmHg}$ LBNP $(524 \pm 182 \mathrm{AP} / \mathrm{min}, 12 \pm 3 \mathrm{AP} / \mathrm{burst}$; main effect LBNP: both $P<0.01$ ). Compared to BSL ( $9 \pm 2$ clusters), $40 \mathrm{mmHg}$ LBNP recruited a subpopulation of previously-silent larger AP clusters $(16 \pm 3$ clusters; $P=0.01)$ while no clusters were recruited from $\mathrm{BSL}$ to $-10 \mathrm{mmHg} \mathrm{LBNP}(10 \pm 1$ clusters; $P=0.31$; LBNP x APN interaction: $P<0.01)$. LBNP did not alter AP latency from BSL $(1.25 \pm 0.03 \mathrm{~s})$, to $-10 \mathrm{mmHg} \mathrm{LBNP}$ $(1.26 \pm 0.03 \mathrm{~s})$, or $-40 \mathrm{mmHg} \operatorname{LBNP}(1.27 \pm 0.05 \mathrm{~s}$; main effect LBNP: $P=0.14)$. Compared to spontaneous breathing, APN 
increased AP discharge when performed at BSL $(\Delta 351 \pm 132 \mathrm{AP} /$ min, $\Delta 5 \pm 2 \mathrm{AP} /$ burst $),-10 \mathrm{mmHg} \mathrm{LBNP}(\Delta 423 \pm 184 \mathrm{AP} / \mathrm{min}$, $\Delta 6 \pm 3 \mathrm{AP} / \mathrm{burst})$, and $-40 \mathrm{mmHg} \operatorname{LBNP}(\Delta 355 \pm 278 \mathrm{AP} / \mathrm{min}$, $\Delta 4 \pm 3$ AP/burst; main effect APN: both $P<0.01)$. APN recruited previously-silent larger AP clusters when performed at BSL $(\Delta 3 \pm 2)$ and $-10 \mathrm{mmHg} \operatorname{LBNP}(\Delta 3 \pm 2 ; P=0.65$ vs. BSL $)$, but not when performed during $-40 \mathrm{mmHg} \operatorname{LBNP}(\Delta 0 \pm 1 ; P=0.02$ vs. BSL; LBNP $\times$ APN interaction: $P<0.01)$. APN decreased AP latency when performed at BSL $(\Delta-0.039 \pm 0.037 \mathrm{~s}),-10 \mathrm{mmHg} \mathrm{LBNP}$ $(\Delta-0.025 \pm 0.027 \mathrm{~s})$, and $-40 \mathrm{mmHg} \operatorname{LBNP}(\Delta-0.030 \pm 0.040 \mathrm{~s}$; main effect APN: $P<0.01)$. The results suggest that apneic stress increases MSNA via rate-coding and population-coding mechanisms unless the subpopulation of previously-silent larger APs is already recruited by baroreflex unloading, at which point the rate-coding mechanism predominates.

Funding: Natural Sciences and Engineering Research Council of Canada (NSERC) Discovery Grant (J.K.S.).

\section{Virtual Poster \#47}

\section{Impact of maternal obesity and sleep apnea on resting muscle sympathetic nerve activity during pregnancy: a pilot study}

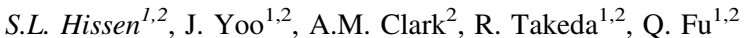

${ }^{1}$ Institute for Exercise and Environmental Medicine at Texas Health Presbyterian Hospital Dallas, Dallas, TX, USA; ${ }^{2}$ University of Texas Southwestern Medical Center, Dallas, TX, USA

Introduction: Maternal obesity is associated with an elevated risk of adverse pregnancy outcomes such as gestational hypertension, preeclampsia, preterm birth, etc. The risk of these adverse outcomes is further increased in women with obstructive sleep apnea (OSA). Pregnancy, obesity and OSA per se are all associated with sympathetic activation. However, it is unknown whether obesity and OSA increase the risk of adverse outcomes through sympathetic neural mechanisms. We hypothesized that obese women have greater sympathetic activation during pregnancy compared with normal weight women, and sympathetic activity is further increased in women with OSA.

Methods: Blood pressure, heart rate and muscle sympathetic nerve activity (MSNA) were measured for $5 \mathrm{~min}$ of supine rest during early pregnancy (6-10 weeks) in 18 normal weight (NWG, BMI $22 \pm 3 \mathrm{~kg} / \mathrm{m}^{2}$ ) and 17 obese (OG, BMI $36 \pm 5 \mathrm{~kg} / \mathrm{m}^{2}$ ) women. 24-h ambulatory blood pressure monitoring was measured. Home-based sleep testing using a portable device was conducted to test for OSA (defined as apnea/hypopnea index of $\geq 5$ ). Comparisons were also made in a subgroup of women (11 NWG, $7 \mathrm{OG})$ during late pregnancy (32-34 weeks).

Results: During early pregnancy, OG had greater levels of 24-h systolic $(127 \pm 13$ vs. $109 \pm 7 \mathrm{mmHg}, \mathrm{p}<0.0001)$ and diastolic (71 \pm 9 vs. $62 \pm 4 \mathrm{mmHg}, \mathrm{p}=0.003$ ) blood pressure when compared with NWG and heart rate tended to be higher in OG ( $83 \pm 9$ vs. $77 \pm 10 \mathrm{bpm}, \mathrm{p}=0.08$ ). There were no significant differences in resting MSNA between OG and NWG $(\mathrm{p}>0.05)$. However, OG with OSA $(n=4)$ exhibited greater levels of MSNA when compared with OG without OSA $(45 \pm 6$ vs. $22 \pm 10$ bursts/min; $58 \pm 13$ vs. $27 \pm 9$ bursts/100 heartbeats; $681 \pm 123$ vs. $271 \pm 147 \mathrm{AU} / \mathrm{min}$ ). During late pregnancy, there were no significant differences in 24-h blood pressure or heart rate between OG and NWG. However, OG had greater levels of resting MSNA burst frequency (49 \pm 16 vs. $24 \pm 11$ bursts $/ \mathrm{min}, \mathrm{p}=0.001)$, burst incidence $(61 \pm 19$ vs. $32 \pm 15$ bursts $/ 100$ heartbeats, $\mathrm{p}=0.002$ ) and total activity $(713 \pm 283$ vs. $350 \pm 140 \mathrm{AU} / \mathrm{min}, \mathrm{p}=0.002)$.
Conclusion: OSA exaggerates sympathetic activation during early pregnancy in obese women and obesity is associated with greater increases in MSNA in late pregnancy when compared with normal weight women. These preliminary findings suggest that obesity and OSA may contribute to the risk of adverse outcomes during pregnancy through sympathetic overactivity.

Funding: Supported by the NIH R01 grant (HL142605).

\section{Virtual Poster \#48}

Elevated cerebral blood flow in individuals with pure autonomic failure

K.R. Hay ${ }^{l}$, P. Trujillo ${ }^{1}$, O.C. Roman ${ }^{2}$, S.Y. Paranjape ${ }^{3,4}$,

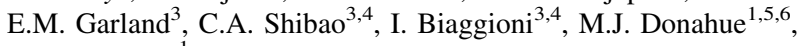
D.O. Claassen ${ }^{1}$

${ }^{1}$ Department of Neurology, Vanderbilt University Medical Center, Nashville, TN, USA; ${ }^{2}$ Vanderbilt University School of Medicine, Nashville, TN, USA; ${ }^{3}$ Department of Medicine, Vanderbilt University Medical Center, Nashville, TN, USA; ${ }^{4}$ Department of Pharmacology, Vanderbilt University Medical Center, Nashville, TN, USA;

${ }^{5}$ Department of Radiology and Radiological Sciences, Vanderbilt University Medical Center, Nashville, TN, USA; ${ }^{6}$ Department of Psychiatry and Behavioral Sciences, Vanderbilt University Medical Center, Nashville, TN, USA

Background: Individuals with pure autonomic failure (PAF) are at high risk for converting to a central nervous system (CNS) alphasynucleinopathy although they can have subclinical pathophysiological profiles of autonomic dysfunction prior to conversion. Central hemodynamic changes in PAF may inform the mechanisms of prodromal stages of central synucleinopathies and represent biomarkers for phenoconversion. Here, cerebral blood flow (CBF) was investigated to test the hypothesis that aberrant $\mathrm{CBF}$ and associated flow profiles are present in PAF participants, relative to healthy, adults. Methods: Individuals with a PAF diagnosis $(\mathrm{n}=17)$ were recruited and evaluated by the Vanderbilt University Medical Center Autonomic Rare Diseases Clinical Research Consortium and normotensive healthy controls $(n=12)$ were recruited from the local community. They were provided informed consent and underwent clinical evaluations, questionnaires, neurological examinations, and 3-Tesla MRI measurements of CBF. CBF maps were also used to examine dural venous sinus hyperintensities, indicative of accelerated capillary flow and reduced oxygen extraction. Non-parametric tests and general linear models were applied to contrast study variables (significance: two-sided $\mathrm{p}<0.05$ ).

Results: Cortical CBF was elevated in PAF $(51.1 \pm 13.4 \mathrm{ml} / 100 \mathrm{~g} /$ min) compared with healthy $(42.0 \pm 6.6 \mathrm{ml} / 100 \mathrm{~g} / \mathrm{min}, \mathrm{p}=0.008)$ participants and CBF was inversely correlated with supine systolic blood pressure (Spearman's-rho $=-0.679, p=0.004$ ) in PAF participants. In PAF participants only, venous hyperintensities were observed and these were over-represented in participants with the highest mean CBF. Such hyperintensities are consistent with rapid erythrocyte transit through capillaries and reduced oxygen extraction efficiency in other populations; here, participants with vs. without these features had UPDRS Part I $(\mathrm{p}=0.038)$, UPDRS Part II $(\mathrm{p}=0.010)$, and UMSARS $(\mathrm{p}=0.021)$ scores, indicating higher motor symptom severity.

Conclusions: Findings support the presence of hemodynamic dysregulation in the periphery and CNS of patients with PAF. Furthermore, the highest CNS dysregulation, manifesting as mild hyperemia and accelerated capillary flow, co-localized with greater motor impairment and may indicate reduced oxygen extraction. 
Ongoing investigations to evaluate how these profiles predict phenoconversion to central synucleinopathies are warranted.

Funding: This study was supported by the National Institutes of Health (R01 NS097783 (to D.O.C), R01 HL122847-01 (to I.B.), R01 HL149386 (to I.B.) and 1UL1 RR000445 (to Vanderbilt University)), the American Heart Association (14GRNT20150004 (to M.J.D) and 19CDA34790002 (to M.R.J.)), and the Food and Drug Administration (FD-R-04778-01-A3 (to S.A.C.)).

\section{Virtual Poster \#49}

\section{Cerebrovascular compliance is regulated by sympathetic and cholinergic inputs}

\section{M.E. Moir ${ }^{1}$, J.W. Hamner ${ }^{2}$, C.O. Tan ${ }^{2,3}$, J.K. Shoemaker ${ }^{1,4}$}

${ }^{1}$ School of Kinesiology, University of Western Ontario, London, ON, Canada; ${ }^{2}$ Cerebrovascular Research Laboratory, Spaulding Hospital Cambridge, Cambridge, MA, USA; ${ }^{3}$ Physical Medicine and Rehabilitation, Harvard Medical School, Boston, MA, USA; ${ }^{4}$ Department of Physiology and Pharmacology, University of Western Ontario, London, ON, Canada

Increasing evidence indicates that cerebrovascular compliance contributes to the dynamic regulation of cerebral blood flow. However, whether sympathetic and cholinergic inputs modify cerebrovascular compliance remains unknown. Cerebrovascular compliance was assessed following sympathetic blockade (Experiment 1) and cholinergic blockade (Experiment 2). Experiment 1 was performed in 11 healthy adults (21-40 years; 4 females) and Experiment 2 in 9 healthy adults (21-30 years; 4 females). Participants completed a 5-min baseline followed by an oscillatory lower body negative pressure (OLBNP) protocol ( -30 to $40 \mathrm{mmHg}$ ) at 6 frequencies $(0.03-0.08 \mathrm{~Hz})$. Following drug infusion (Experiment 1: phentolamine to block $\alpha$-adrenergic effects on the cerebral vasculature; Experiment 2: glycopyrrolate to block muscarinic receptors on the cerebrovascular endothelium), baseline and OLBNP were repeated. Individual blood pressure (BP) waveforms (Finometer) and corresponding middle cerebral artery blood velocity (BV) waveforms (transcranial Doppler) were extracted (10 beats at baseline and 30 beats during $0.03 \mathrm{~Hz}$ OLBNP) and input into a four-element Windkessel model that quantified indexes of cerebrovascular compliance (Ci) and cerebrovascular resistance (CVRi) (Experiment 1: $\mathrm{n}=9$; Experiment 2: $\mathrm{n}=9$ ). Experiment 1: Phentolamine increased $\mathrm{Ci}$ at baseline $\left(3.8 \mathrm{e}^{-4} \pm 1.5 \mathrm{e}^{-4}\right.$ to $\left.7.2 \mathrm{e}^{-4} \pm 4.0 \mathrm{e}^{-4}\right)$ and during $0.03 \mathrm{~Hz}$ OLBNP $\left(3.1 \mathrm{e}^{-4} \pm 6.7 \mathrm{e}^{-5}\right.$ to $7.7 \mathrm{e}^{-4} \pm 5.8 \mathrm{e}^{-4}$; drug: $P=0.02$, condition: $P=0.88$, interaction: $P=0.43)$. There was no effect of phentolamine infusion on CVRi (drug: $P=0.92$, condition: $P=0.37$, interaction: $P=0.28$ ), mean BP (drug: $P=0.61$, condition: $P=0.50$, interaction: $P=0.16$ ), or mean $\mathrm{BV}$ (drug: $P=0.31$, condition: $P=0.26$, interaction: $P=0.54$ ). Experiment 2: Glycopyrrolate increased $\mathrm{Ci}$ at baseline $\left(2.8 \mathrm{e}^{-4} \pm 1.4 \mathrm{e}^{-4}\right.$ to $\left.4.2 \mathrm{e}^{-4} \pm 1.9 \mathrm{e}^{-4}\right)$ and during $0.03 \mathrm{~Hz}$ OLBNP $\left(2.4 \mathrm{e}^{-4} \pm 9.1 \mathrm{e}^{-5}\right.$ to $3.5 \mathrm{e}^{-4} \pm 2.5 \mathrm{e}^{-4}$; drug: $P=0.05$, condition: $P=0.01$, interaction: $P=0.65)$. Also, CVRi (drug: $P=0.04$, condition: $P=0.32$, interaction: $P=0.45$ ) and mean BP (drug: $P=0.02$, condition: $P=0.29$, interaction: $P=0.53$ ) were increased following glycopyrrolate infusion but mean $\mathrm{BV}$ was unchanged (drug: $P=0.71$, condition: $P=0.89$, interaction: $P=0.96)$. Sympathetic and cholinergic blockade augmented $\mathrm{Ci}$ at baseline and during $0.03 \mathrm{~Hz}$ OLBNP. Sympathetic blockade increased $\mathrm{Ci}$ independent of $\mathrm{CVRi}$ and mean $\mathrm{BP}$ while cholinergic blockade increased Ci, CVRi, and mean BP. Therefore, adrenergic and muscarinic mechanisms affect cerebrovascular compliance.

Funding: This work was supported by a Natural Sciences and Engineering Research Council of Canada (NSERC) Discovery Grant
(RGPIN-2018-06255) and a Canadian Institutes of Health Research (CIHR) Grant (201503MOP-342412-MOVCEEA) awarded to J.K.S.

\section{Virtual Poster \#50}

Impact of patisiran, an RNAi therapeutic, on diarrhea symptoms in patients with hereditary transthyretin-mediated amyloidosis

L. Obici ${ }^{1}$, A. González-Duarte ${ }^{2}$, M. Waddington-Cruz ${ }^{3}$, Q. Dinh ${ }^{4}$,

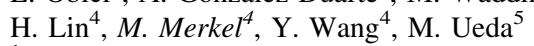

${ }^{1}$ Fondazione IRCCS Policlinico San Matteo, Pavia, Italy; ${ }^{2}$ Instituto Nacional de Ciencias Médicas y Nutrición, Salvador Zubirán, Mexico City, Mexico; ${ }^{3}$ Hospital Universitario Clementino Fraga Filho, Rio de Janeiro, Brazil; ${ }^{4}$ Alnylam Pharmaceuticals, Cambridge, MA, USA;

${ }^{5}$ Kumamoto University Hospital, Kumamoto, Japan

Hereditary transthyretin-mediated (hATTR) amyloidosis is a rapidly progressive disease often resulting in wasting, weight loss, and reduced quality of life. This is due in part to debilitating diarrhea caused by amyloid deposition in the gastrointestinal tract and autonomic nerves. Inanition associated with progressive peripheral or autonomic neuropathy is a leading cause of death among these patients. In the Phase 3 APOLLO study, patisiran demonstrated improvements on dysautonomia as measured by COMPASS-31 and Norfolk QOL-DN. This analysis further evaluates the impact of patisiran on diarrhea. APOLLO was an international, randomized (2:1), double-blinded, placebo-controlled study of patisiran $0.3 \mathrm{mg} / \mathrm{kg}$ or placebo IV $\mathrm{q} 3 \mathrm{~W}$ in patients with hATTR amyloidosis with polyneuropathy (NCT01960348). Change in presence and severity of diarrhea symptoms was evaluated descriptively using question-level analyses from COMPASS-31 and Norfolk QOL-DN. APOLLO enrolled 225 patients: median age 62 years, $74 \%$ male, $43 \%$ V30M, FAP Stage $1(46 \%)$ and $2(53 \%)$. At baseline, two-thirds reported mild to severe bouts of diarrhea in the prior year on COMPASS- 31 . After 18 months, patisiran-treated patients were 3.5 -fold more likely to report improvement in diarrhea compared to placebo patients (18\% vs. $5 \%$, respectively). Patients treated with patisiran were also more likely to remain stable in their diarrhea severity than those receiving placebo (54\% vs. $42 \%$, respectively). On Norfolk QOL-DN, more placebo-treated patients progressed to moderate or severe diarrhea and/or loss of bowel control at 18 months vs. baseline (43\% vs. $33 \%$, respectively); fewer patisiran-treated patients had moderate or severe symptoms at 18 months vs. baseline ( $27 \%$ vs. $34 \%$, respectively). Following 18 months, patisiran was more likely to improve or stabilize diarrhea at a lower-grade severity than placebo based on question-level analyses of patient-reported questionnaires. These data reinforce the clinical benefit of patisiran in addressing the debilitating autonomic symptoms of hATTR amyloidosis.

Funding: Alnylam Pharmaceuticals.

\section{Virtual Poster \#51}

Quantifiable gastrointestinal dysmotility in idiopathic autonomic neuropathy

J. Langford ${ }^{1}$, L. Millsap ${ }^{1}$, L.A. Pace ${ }^{2}$, J. Hemp ${ }^{3}$, M.M. Cortez ${ }^{1}$ ${ }^{1}$ Department of Neurology, University of Utah, Salt Lake City, Utah, USA; ${ }^{2}$ Center for Genomic Medicine, University of Utah, Salt Lake City, UT, USA; ${ }^{3}$ Department of Internal Medicine, University of Utah, Salt Lake City, UT, USA

Autonomic neuropathy (AN) patients frequently present with symptoms affecting multiple organ systems, ranging from cardiovascular, 
sensory, urinary, secretory, and gastrointestinal (GI). While it is well known that autonomic innervation plays an important role in GI motility and secretions, there is marked heterogeneity among AN patients, often limiting the yield of conventional diagnostic evaluations. The aim of this study was to describe our single-institution experience with wireless motility capsule (WMC) testing to quantitatively evaluate segmental dysfunction in AN patients. We performed a retrospective analysis of AN patients that presented with undiagnosed GI and generalized autonomic symptoms, who were seen by both Neurogastroenterology and Autonomic Neurology services at the University of Utah $(2015-2019 ; n=86)$. Inclusion criteria-diagnosis of AN based on $\geq 1$ of the following, abnormal: heart rate variability, sudomotor function, intraepidermal nerve density, and/or sensory thresholds of the initial cohort, 29 of 86 subjects met inclusion criteria. WMC results were analyzed to identify abnormalities in gastric emptying and small bowel/colonic/global transit times. Of the 29 subjects included, 25 were female and 4 male; age range 17-62 years (median 29); 24 had limited AN, whereas 5 had a primary diagnosis of AN. COMPASS-31 scores were available in 25/29, with a mean total score of 54.4 and GI domain score of 15.6. Overall, $25 / 29$ subjects ( $86 \%$ ) had dysmotility in at least one segment of the GI tract, with an average of 2.0 segments affected. Abnormal gastric emptying, was the most common finding $(20 / 29$; 69\%), followed by abnormal global transit time $(18 / 29 ; 62 \%)$, abnormal small bowel transit times $(10 / 29 ; 34 \%)$ and colonic transit times $(9 / 29$; $31 \%$ ). In this cohort of 29 subjects with $\mathrm{AN}$ and prominent undiagnosed GI symptoms, $86 \%$ had objectively quantifiable abnormal segmental WMC testing. These data support the likelihood that AN patients with prominent GI symptoms also have measurable changes in GI motility, reflective of possible visceral involvement of their autonomic neuropathy. These findings establish GI dysmotility as a key feature of AN with GI symptoms, and merit future studies to further evaluate underlying pathophysiology, including enteric nerve density in cases of otherwise unexplained visceral involvement of autonomic neuropathies.

Funding: LAP, Dysautonomia International, Grant No. DI 10049068 and National Institutes of Health, Office of Research on Women's Health, Grant No. K12HD085852.

\section{Virtual Poster \#52}

\section{Gastrointestinal dysmotility is common in autonomic nervous system disorders and has important implications for clinical management}

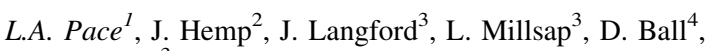
M.M. Cortez ${ }^{3}$

${ }^{1}$ Center for Genomic Medicine, University of Utah, Salt Lake City, UT, USA; ${ }^{2}$ Department of Internal Medicine, University of Utah, Salt Lake City, UT, USA; ${ }^{3}$ Department of Neurology, University of Utah, Salt Lake City, UT, USA; ${ }^{4}$ Division of Physical Medicine and Rehabilitation, Department of Internal Medicine, Division of Medical Genetics, Department of Pediatrics, University of Utah, Salt Lake City, UT, USA

Gastrointestinal (GI) symptoms are common in individuals with autonomic nervous system (ANS) disorders. We analyzed 106 patients with formalized gastrointestinal motility testing via wireless motility capsule (WMC) for presence of a comorbid ANS disorder. A total of 86 patients underwent both WMC and formalized ANS testing. $87.2 \%$ were found to have a dysautonomia $(50.7 \%$ postural orthostatic tachycardia syndrome (POTS), 29.3\% orthostatic intolerance (OI), $6.7 \%$ primary autonomic neuropathy, $14.7 \%$ other). $89.5 \%$ of POTS patients had GI-dysmotility with an average of 1.8 regions affected. $95.5 \%$ of OI patients had GI-dysmotility with an average of 2.3 regions affected. $100 \%$ of patients with a primary autonomic neuropathy had GI-dysmotility with an average 2.8 regions affected. $81.2 \%$ of other ANS disorders had GI-dysmotility. $81.8 \%$ of patients with negative ANS testing had evidence of GI-dysmotility with an average of 1.27 regions affected and $75 \%$ of those not tested for an ANS disorder had GI-dysmotility with an average of 1.9 regions affected. Sixty-three patients had documented COMPASS-31 scores; $100 \%$ had a score $>9.6$ and $95.2 \%$ had a score $>25.8$, (two standard-deviations above normal). $87.3 \%$ had GI-dysmotility and a weighted GI domain score $>$ 9.6. COMPASS-31 scores may serve as good screening tools to identify individuals with GI-dysmotility and ANS disorders. Given the high concordance rates for GI-dysmotility and ANS disorders, these findings strongly support the need for thorough GI evaluations for individuals with known ANS disorders and gastrointestinal symptoms and thorough ANS evaluations for individuals with otherwise idiopathic GI-dysmotility. The identification of GI-dysmotility in patients with ANS disorders will be important for developing appropriate management strategies as GIdysmotility will likely impact an individuals' ability to comply with standard salt and fluid recommendations. Furthermore, GI motility testing has the potential to expand our ability to interrogate the functioning of the ANS and should be considered as part of the ANS evaluation moving forward. In conclusion, objective gastrointestinal dysmotility is common in ANS disorders, including POTS and OI. This data serves to highlight the danger of classifying POTS/OI as functional disorders given the degree of objective GI-dysmotility in these patients and the clinical impact of these findings.

Funding: Dysautonomia International, Grant No. DI 10049068 and National Institutes of Health, Office of Research on Women's Health, Grant No. K12HD085852 (to LAP).

\section{Virtual Poster \#53}

Orthostatic blood pressure and arterial stiffness in persons with spinal cord injury: the effect of the renin-angiotensinaldosterone system

C.G. Katzelnick ${ }^{1,2,3}$, J.P. Weir ${ }^{4}$, G.P. Zipp ${ }^{3}$, M.F. LaFountaine ${ }^{1,5,6}$, J.M. Wecht ${ }^{1,7}$

${ }^{1}$ James J Peters VA Medical Center, Bronx, NY, USA; ${ }^{2}$ Kessler Foundation, West Orange, NJ, USA; ${ }^{3}$ Department of Interprofessional Health Sciences and Health Administration, School of Health and Medical Sciences, Hackensack Meridian School of Medicine at Seton Hall University, Nutley, NJ, USA; ${ }^{4}$ University of Kansas, Lawrence, KS, USA; ${ }^{5}$ Department of Physical Therapy, School of Health and Medical Sciences, Seton Hall University, South Orange, NJ, USA; ${ }^{6}$ Departments of Medical Sciences and Neurology, Hackensack Meridian School of Medicine at Seton Hall University, Nutley, NJ, USA; ${ }^{7}$ Icahn School of Medicine, Mount Sinai, New York, NY, USA

Objective: The purpose of this study was to determine the relationship between pulse wave velocity (PWV) and orthostatic changes in BP, norepinephrine (NE) and plasma renin in individuals with spinal cord injury (SCI) and age-matched controls.

Participants: Participants included 11 individuals with cervical (CERVICAL; C4-T1), 11 individuals thoracic (THORACIC; T6T12) SCI, and 11 non-SCI controls (Controls).

Methods: Participants were transferred to the tilt table and remained in supine position for 30-min prior to collection of hemodynamic data including BP, PWV, and a blood draw for renin and NE. Participants were then progressively tilted from supine to $30^{\circ}, 45^{\circ}$ and $60^{\circ}$ for $10 \mathrm{~min}$ at each tilt. A second blood draw was collected at the end of the $60^{\circ}$ tilt. 
Results: There were significant group differences in PWV $\left(\mathrm{F}_{2,30}=12.68, \mathrm{p}<0.001, \eta^{2}=0.46\right)$; Post hoc analyses indicated that PWV was significantly increased in CERVICAL $(8.81 \pm 1.91 \mathrm{~m} / \mathrm{s})$ and THORACIC $(7.36 \pm 1.58 \mathrm{~m} / \mathrm{s})$ SCI groups compared to controls $(5.53 \pm 0.95 \mathrm{~m} / \mathrm{s} ; \mathrm{p}<0.05)$. There were no significant group differences in supine systolic BP (SBP); however, the group differences were significant at $60^{\circ}\left(p=0.0014, \eta^{2}=0.36\right)$, such that SBP in was lower in the CERVICAL $(94.86 \pm 20.33 \mathrm{mmHg})$ compared to THORACIC $(125.03 \pm 14.42 \mathrm{mmHg} ; \quad \mathrm{p}<0.05)$ and control groups $(128.07 \pm 26.96 \mathrm{mmHg} ; \mathrm{p}<0.05)$. The interaction effects (group, tilt angle) were significant for both renin $\left(p=0.005, \eta^{2}=0.296\right)$ and $\mathrm{NE}\left(\mathrm{p}=0.016, \eta^{2}=0.242\right)$. Change in renin was significantly increased in CERVICAL $(11.54 \pm 12.29 \mathrm{pg} / \mathrm{mL})$ compared to THORACIC $\quad(0.47 \pm 5.67 \mathrm{pg} / \mathrm{mL} ; \quad \mathrm{p}<0.05)$ and controls $(1.22 \pm 3.45 \mathrm{pg} / \mathrm{mL} ; \mathrm{p}<0.05)$. Change in $\mathrm{NE}$ was significantly reduced in CERVICAL $(54.27 \pm 68.64 \mathrm{pg} / \mathrm{mL})$ compared to THORACIC $(203.73 \pm 171.88 \mathrm{pg} / \mathrm{mL}, \mathrm{p}<0.05)$. Both SCI group and change in renin were significant contributors to the prediction of PWV $\left(\mathrm{R}^{2}=0.63, \mathrm{p}=0.001\right)$.

Conclusion: These preliminary findings suggest that orthostatic hypotension and increased plasma renin responses to upright positioning contribute to elevated PWV, a marker of arterial stiffness, in otherwise healthy individuals with SCI.

Funding: New Jersey Commission on Spinal Cord Research (\#CSCR18FEL010) and VA RR\&D Service (\#B2020-C).

\section{Virtual Poster \#54}

\section{Frequency of injuries associated with syncope in the prevention} of syncope trials

J.G. Jorge*, P. Pournazari*, S.R. Raj, C. Maxey, R.S. Sheldon Libin Cardiovascular Institute of Alberta, Department of Cardiac Sciences, University of Calgary, Calgary, Alberta, Canada; *J.G.J and P.P. contributed equally to this project

Aims: Syncope is a common clinical problem and can cause injuries. The likelihood and severity of injuries due to a syncopal spell is not well understood. We determined the frequency, severity, and predictors of injuries due to syncope in cohorts of syncope patients. Methods: Participants were enrolled in the POST2 (fludrocortisone) and POST4 (midodrine) vasovagal syncope (VVS) randomized trials, and patients in POST3 had bifascicular block and syncope. Injury was defined as minor (bruising, abrasions), moderate (lacerations), and severe (fractures, burns, joint pain), and documented up to 1 year after enrollment.

Results: A total of 459 patients (median 39 years old) were analysed. A total of 710 faints occurred in 186 patients during a 1-year followup. Fully 56/186 (30\%) of patients were injured with syncope (12\% of overall group). There were 102 injuries associated with the 710 faints (14\%), of which $19 \%$ were moderate or severe injuries. Females with syncope were more likely to be injured, having a lower injury-free survival at 1 year than males $(65 \%$ vs. $85 \% ; \mathrm{p}=0.03)$. Patients with bifascicular block were more prone to injury (Relative Risk 1.98, $\mathrm{p}=0.018)$. Patients with $\geq 4$ faints in the prior year had more injuries than those with fewer faints (Relative Risk 2.97, $\mathrm{p}<0.0001$ ), but this was due to more frequent syncope, and not more injuries per faint. In VVS patients, pharmacological therapy significantly reduced the likelihood of an injury due to a syncopal spell (Relative Risk 0.64, $p=0.015$ ). Injury severity did not associate with age, sex or prior year syncope frequency.

Conclusion: The frequency of injuries is significant in patients with syncope, although most are contusions and abrasions. Only $4 \%$ of injuries were severe. Patients and physicians should be alert to the consequences of recurrent syncope and injuries and that females and patients with frequent syncope are more likely to be injured.

\section{Virtual Poster \#55}

\section{Characterizing the physiology of an active stand in initial orthostatic hypotension}

N. Sheikh, S. Ranada, M. Lloyd, D. McCarthy, R.S. Sheldon, A. Phillips, D.V. Exner, M. Runte, S.R. Raj

Department of Cardiac Sciences, Libin Cardiovascular Institute, University of Calgary, Calgary, Alberta, Canada

Background: Initial orthostatic hypotension ( $\mathrm{IOH})$ is a common form of orthostatic intolerance characterized by a reduction in blood pressure almost immediately on standing, with rapid recovery. It is thought that an initial large muscle activation reflex results in rapid vasodilation. When patients sit down and then stand almost immediately, the symptoms typically do not reappear, suggesting that the reflex might have a refractory period. We hypothesized that drops in systolic blood pressure (SBP) and systemic vascular resistance (SVR) are less after a SHORT (30 s) versus a LONG (20 min) sit.

Methods: Patients with IOH performed sit-to-stand maneuvers with varying seated baseline durations: a SHORT and a LONG sit. The order was randomized. Continuous heart rate (HR), SBP, diastolic blood pressure (DBP), SVR, stroke volume (SV) and cardiac output (CO) were recorded. Data are presented as mean \pm standard error of the mean (SEM).

Results: A total of 24 female IOH subjects ( $32 \pm 2$ years; body mass index $24 \pm 1 \mathrm{~kg} / \mathrm{m}^{2}$ ) were enrolled. The drops in SBP and DBP following the SHORT sit $(11 \pm 2 \mathrm{mmHg}$ and $7 \pm 1 \mathrm{mmHg})$ were reduced compared to the LONG sit $(34 \pm 5.1 \mathrm{mmHg}$ and $24 \pm 3 \mathrm{mmHg}), \mathrm{p}<0.001$. The drop in SVR following the SHORT sit $\left(296.4 \pm 35.5\right.$ dynes s cm$\left.{ }^{-5}\right)$ was reduced compared to the LONG sit $\left(521.3 \pm 58.3\right.$ dynes $\left.\mathrm{s} \mathrm{cm}^{-5}\right), \mathrm{p}<0.001$. There were blunted increases in HR and CO following the SHORT sit $(13 \pm 2 \mathrm{bpm}$, $2 \pm 0.3 \mathrm{~L} / \mathrm{min})$ compared to the LONG sit $(29 \pm 3 \mathrm{bpm}, 3 \pm 0.4$ $\mathrm{L} / \mathrm{min}), \mathrm{p}<0.001$ and $\mathrm{p}=0.002$ respectively. The time to nadir blood pressure $(8 \pm 1 \mathrm{~s} ; \mathrm{p}=\mathrm{NS})$ and SV $(17 \pm 3 \mathrm{ml} ; \mathrm{p}=\mathrm{NS})$ were not different between the two stands.

Conclusion: These data suggest that the SBP drop seen in IOH is associated with a drop in SVR. Increases in HR and CO were blunted in response to the reduced drop in SBP and SVR following the SHORT sit. The standing-induced reductions in SBP and SVR were significantly mitigated following the $30 \mathrm{~s}$ sit compared to the $20 \mathrm{~min}$ sit, suggesting that there is a refractory period to this hemodynamic reflex.

\section{Virtual Poster \#56}

Midodrine improves quality of life in patients with vasovagal syncope: results from the Prevention of Syncope Trial IV

L.Y. Lei, R.S. Sheldon, S. Safdar, D. Ritchie, C. Maxey, S.R. Raj Department of Cardiac Sciences, Libin Cardiovascular Institute, University of Calgary, Calgary, AB, Canada

Background: Recurrent vasovagal syncope (VVS) is a common clinical problem that is associated with significantly impaired healthrelated quality of life (HRQoL). Current guidelines suggest that midodrine might suppress VVS, but its ability to provide QoL improvement is largely unknown. 
Methods: The EuroQol-5D (EQ-5D; 0-100, lower scores indicate poorer generic HRQoL [worse]) and Impact of Syncope on Quality of Life (ISQL; 0-55, lower scores indicate less impact of syncope on HRQoL [better]) scales were completed at baseline and 6 months post-enrollment by VVS patients in the Prevention of Syncope Trial IV (POST4), a randomized, placebo-controlled trial of midodrine. Differences in HRQoL were compared by randomization group and syncope recurrence status at 6 months.

Results: Complete survey data was available for $63 \mathrm{VVS}$ patients (38 \pm 14 years; $70 \%$ female; 5 syncopal episodes in the preceding year [median]). There were no significant baseline differences in HRQoL between the placebo and midodrine groups. At 6 months, the midodrine group reported superior $\mathrm{HRQ}$ oL relative to the placebo group (ISQL: 17 vs. $24, p=0.04$; EQ-5D: 80 vs. $69, p<0.01$ ). With midodrine, both syncope-specific and generic HRQoL had significantly improved at follow-up compared to baseline (ISQL: 17 vs. 24, $p=0.02$; EQ-5D: 80 vs. $73, p=0.03$ ). Patients in this treatment group reported similar levels of HRQoL at 6 months regardless of whether they did or did not experience syncope during follow-up (ISQL: 18 vs. $16, p=0.61$; EQ-5D: 82 vs. $79, p=0.61$ ). Conversely, in the placebo group, while syncope-specific HRQoL had improved by 6 months (ISQL: 30 vs. $24, p=0.02$ ), there was no change in generic HRQoL (EQ-5D: 68 vs. 69, $p=0.76$ ). Additionally, patients in the placebo group that had at least one syncopal episode reported poorer HRQoL compared to those who were syncope-free during the 6-month period (ISQL: 32 vs. 20, $p=0.04$; EQ-5D: 59 vs. 76, $p<0.01)$.

Conclusion: Over time, midodrine improves HRQoL in VVS patients relative to placebo, even with syncope recurrence during follow-up. Improvements with placebo may be attributable to "placebo effect" or interaction with experts during clinical trial enrollment.

\section{Virtual Poster \#57}

\section{Transient early orthostatic hypotension: a subtype of orthostatic hypotension}

\section{P. Shekhawat, K. Senechal, V. Galvis, I. Bonyhay, C. Gibbons, R. Freeman \\ Department of Neurology, Beth Israel Deaconess Medical Center, Harvard Medical School, Boston, MA, USA}

Background: Orthostatic hypotension $(\mathrm{OH})$ is defined as a sustained reduction in systolic blood pressure (SBP) of $\geq 20 \mathrm{~mm} \mathrm{Hg}$ or diastolic BP (DBP) of $\geq 10 \mathrm{~mm} \mathrm{Hg}$ within 3 min of active standing or tilt-table test. Some patients have a transient reduction in blood pressure within 3 min of active standing and/or tilt with recovery. We classify this finding as transient early orthostatic hypotension (TEOH). The clinical significance of this observation is not known. Objective: To investigate the neurophysiologic features and clinical consequences of TEOH.

Methods: We reviewed medical records of 432 individuals referred for autonomic testing. BP was recorded at 1-min intervals during $60^{\circ}$ head-up tilt for $45 \mathrm{~min}$ and standing for $5 \mathrm{~min}$. TEOH was defined as a SBP decrease $\geq 20 \mathrm{mmHg}$ by cuff sphygmomanometer during the first $3 \mathrm{~min}$, with recovery within $5 \mathrm{~min}$ of active standing or $10 \mathrm{~min}$ of tilt-table test. Beat-to-beat BP, tests of cardiovagal function and sympathetic-adrenergic function were performed. Heart rate variability to paced breathing (max-min and E:I ratio) and the Valsalva ratio (VR) were calculated. The SBP fall during phase II and increase in phase IV of the Valsalva maneuver were calculated.

Results: $61 \mathrm{TEOH}$ patients (59 \pm 15 years, $59 \%$ female) were studied. Of these, $26 \%$ had TEOH on both stand and the tilt-table-test; $54 \%$ on stand alone; and $20 \%$ on the tilt-table test alone. Patients with
TEOH on stand test tended to have a lower E:I ratio $(\mathrm{p}<0.01)$, max$\min (\mathrm{p}<0.01)$, VR $(\mathrm{p}<0.02)$, and a phase IV overshoot $(\mathrm{p}<0.01)$ when compared to the controls with normal autonomic test results. The difference in these variables did not reach statistical significance for patients with TEOH on the tilt-table test alone when compared to the controls.

Conclusion: TEOH occurred in $14 \%$ of our tested population and was associated with abnormalities of parasympathetic and sympathetic adrenergic function, suggesting that TEOH may be a mild or early form of autonomic failure.

\section{Virtual Poster \#58}

Effect of the initial maintenance dose of droxidopa on treatment persistence in patients with neurogenic orthostatic hypotension

F. Amjad ${ }^{1}$, L.A. Hewitt ${ }^{2}$, S. Kymes ${ }^{3}$, B. Polenchar ${ }^{4}$, A. Favit ${ }^{2}$ ${ }^{1}$ Department of Neurology, Georgetown University Hospital Pasquerilla Healthcare Center, Washington, DC, USA; ${ }^{2}$ Medical Affairs, Lundbeck, Deerfield, IL, USA; ${ }^{3}$ Health Economics and Outcomes Research, Lundbeck, Deerfield, IL, USA; ${ }^{4}$ Business Insights, Lundbeck, Deerfield, IL, USA

Objective: To examine titration schedules, daily doses, and treatment persistency of droxidopa in patients with neurogenic orthostatic hypotension $(\mathrm{nOH})$ treated in clinical practice settings.

Background: Treatment with droxidopa (approved to treat $\mathrm{nOH}$ symptoms) requires titration to an individualized effective dose (100-600 mg 3 times daily [tid]). Per the product label, droxidopa should be titrated every $24-48$ h to the optimum maintenance dose, based on symptomatic response, up to a maximum total daily dose of $1800 \mathrm{mg}$ (600 mg tid). In clinical trials, patients were titrated to mean daily droxidopa doses of $1167-1404 \mathrm{mg} ; 82 \%$ received a total daily dose $\geq 900 \mathrm{mg}$.

Methods: Patient data were collected from the central NORTHERA ${ }^{\circledR}$ specialty pharmacy hub. Average daily titration doses and maintenance doses were calculated from total drug dispensed on the first 30-day dispense (i.e., assumed titration period) and subsequent dispenses (i.e., assumed post-titration), respectively (total milligrams/days supplied). The relationship between maintenance dose and treatment persistence was examined by number of refills (used as a surrogate for persistence). Discontinuation was defined as no refill within 30 days of expected run-out.

Results: $53 \%$ of patients were titrated with custom schedules (i.e., different from the product labeling recommendation) and had an average daily titration dose of $567 \mathrm{mg} ; 47 \%$ were titrated according to the product label schedule (48-h, 37\%; 24-h, 10\%) and had daily titration doses of $1500-1650 \mathrm{mg}$. The average daily maintenance doses in patients who received 2, 3-6, 7-24, and $>25$ dispenses were $938 \mathrm{mg}, 969 \mathrm{mg}, 1069 \mathrm{mg}$, and $1167 \mathrm{mg}$, respectively $(P<0.0001)$. Conclusion: In this analysis of real-world practice, more than half of patients treated with droxidopa were titrated differently from the schedule recommended in the product label (i.e., not 24-48 h) and received lower daily doses of droxidopa than those treated using recommended titration schedules. A lower daily maintenance dose of droxidopa was associated with shorter treatment persistence (i.e., fewer dispenses), while patients who were titrated to higher dosage received more follow-up dispensing. Reasons for discontinuation could not be examined in this study, but further investigation of these persistency data is warranted.

Funding: Lundbeck. 


\section{Virtual Poster \#59}

\section{A comparison of the Active Standing Test (AST) and Head-Up Tilt Test (HUTT) in children and young people}

\author{
K. Vakili ${ }^{1}$, D. Moore ${ }^{2}$, G. Parker ${ }^{1}$, W.P. Whitehouse $e^{1,3}$ \\ ${ }^{1}$ Department of Paediatric Neurology, Nottingham University \\ Hospitals NHS Trust, Nottingham, UK; ${ }^{2}$ Department of Clinical \\ Neurophysiology, Nottingham University Hospitals NHS Trust, \\ Nottingham, UK; ${ }^{3}$ School of Medicine, University of Nottingham, \\ Nottingham, UK
}

Background: The Head-Up Tilt Test (HUTT) is an established autonomic function test for identifying disorders of blood pressure (BP) and heart rate (HR) regulation. It can demonstrate abnormalities comprising excessive falls in BP and or HR, as well as excessive postural tachycardia (PT). The Active Standing Test (AST) is quicker and easier to apply, e.g., in an office or busy out-patient setting, and may be more sensitive in demonstrating PT. We compared the results obtained from patients routinely undergoing a $10 \mathrm{~min}$ AST immediately before their $45 \mathrm{~min} 60^{\circ} \mathrm{HUTT}$.

Methods: This was a retrospective clinical notes review of unselected consecutive children and young people undergoing HUTT immediately preceded by an AST. This was part of a registered clinical audit of our HUTT procedures.

Results: $12 / 84(14 \%)$ children and young people with complete data sets for the first $10 \mathrm{~min}$ of HUTT and $10 \mathrm{~min}$ AST had a significant drop in BP and or HR on HUTT. Only $1 / 12$ positive on HUTT was also positive on AST. However, an additional 6/84 (7\%) were positive on AST but not on HUTT, giving 18/84 (21\%) positive in total. 8/86 (9\%) children and young people with complete HR data sets for the first $10 \mathrm{~min}$ of HUTT and $10 \mathrm{~min}$ AST had a significant rise in HR on HUTT. Only $3 / 8$ positive on HUTT were also positive on AST. However, an additional 10/86 (12\%) were positive on AST but not on HUTT, giving 18/86 (21\%) positive in total. While HUTT yielded more cases with significant falls in BP and/or HR than AST (14\% vs. $8 \%$ ), combining the tests gave the highest yield (21\%). While AST yielded more cases with significant rises in HR than HUTT (15\% vs. $9 \%)$, combining the tests gave the highest yield $(21 \%)$.

Conclusions: We recommend routinely undertaking a 10 min AST prior to the $45 \mathrm{~min} 60^{\circ} \mathrm{HUTT}$ in children and young people.

\section{Virtual Poster \#60}

\section{Likelihood of injury due to vasovagal syncope. A systematic review and meta-analysis}

\section{J.G. Jorge, S.R. Raj, R.S. Sheldon}

Department of Cardiac Sciences, Libin Cardiovascular Institute of Alberta, Cumming School of Medicine, University of Calgary, Calgary, AB, Canada

Background: Vasovagal syncope is the most common type of syncope and is usually considered a benign disorder. The likelihood of injury is worrisome but unknown.

Objectives: To determine the proportion of patients with injuries due to vasovagal syncope.

Methods: A systematic search of relevant studies, published until September 2019, was performed in multiple medical and nursing databases. We included studies with data on the proportion of patients with physical injury due to vasovagal syncope prior to study enrollment. The Random effects methods were used.

Results: Eighteen studies (2916 patients; 55\% female; mean 51 years) met inclusion criteria. All patients were diagnosed clinically with vasovagal syncope and $92 \%$ had at least one syncope in the 2 years prior to study enrollment. Head-up tilt tests were positive in $68 \%$ of patients, and in 11 studies $35 \%$ of 2029 patients had hypertension. The weighted mean risk of injury was $38 \%$ (CI 31\%, 44\%; $\mathrm{p}<0.001)$. The likelihood of injury correlated with population age $(r=0.4, p=0.03)$, but not with sex, tilt test outcome, or hypertension. The injury rates in studies with younger patients mean age $\leq 50$ years $(\mathrm{n}=1225)$ were $29 \%(\mathrm{CI} 21 \%, 40 \%)$ compared to $46 \%($ CI $40 \%, 52 \%)$ in studies with patients $>50$ years $(n=1691)$. The number of lifetime syncopal episodes did not associate with the likelihood of injury: the likelihood of injury rates in studies $(\mathrm{n}=1289$, mean age 53 years $)$ with more than 6 lifetime syncopal episodes were $29 \%$ (CI 16\%, 46\%) compared to $34 \%$ (CI 26\%, 44\%) in studies with 6 or fewer lifetime syncopal episodes $(n=804$, mean age 47 years). Seven studies specifically reported major injuries; the weighted mean likelihood of major injuries was 13\% (CI 9\%, 20\%). Conclusion: Injuries due to syncope are frequent, occurring in $38 \%$ of patients with a history of vasovagal syncope. The risk of injury is substantial, and major injuries are common. Older patients are at considerably higher risk. Clinicians should be aware of the substantial risk of injuries when providing care and advice to patients with vasovagal syncope.

\section{Virtual Poster \#61}

\section{Impact of orthostatic hypotension in inpatient rehabilitation}

P. Sharma ${ }^{1}$, L.E. Okamoto ${ }^{1}$, L. Massey ${ }^{2}$, I. Biaggioni ${ }^{1}$, J. Johns ${ }^{2}$ ${ }^{1}$ Department of Medicine, Division of Clinical Pharmacology, Vanderbilt University Medical Center, Nashville, TN, USA; ${ }^{2}$ Physical Medicine and Rehabilitation, Vanderbilt Stallworth Rehabilitation Hospital, Nashville, TN, USA

Inpatient rehabilitation is crucial in the management of patients after injuries, surgery, or those with debilitating or neurological conditions. Orthostatic hypotension $(\mathrm{OH})$ is commonly associated with these conditions, and therefore, the inability to stand and participate in rehabilitation therapy can negatively impact their recovery. To test this hypothesis, we study a cohort of 8352 patients who underwent rehabilitation at Vanderbilt Stallworth Rehabilitation Hospital between 2014 and 2019 to assess the prevalence of $\mathrm{OH}$ and its impact on the length of stay (LOS), functional independence measure (FIM) change (discharge FIM-admission FIM), and FIM efficiency (FIM change/LOS), compared to patients without $\mathrm{OH}$ admitted during the same period. We found that the prevalence of $\mathrm{OH}$ was $3.8 \%(n=314$; $60 \pm 18$ years; 227 men). $\mathrm{OH}$ was commonly associated with patients admitted for neurological conditions $(29 \%, \mathrm{n}=91)$, spinal cord dysfunction $(22 \%, \mathrm{n}=71)$, orthopedic disorders $(13 \%, \mathrm{n}=13)$, and stroke $(10 \%, \mathrm{n}=33)$. On admission, patients with $\mathrm{OH}$ had a longer predicted LOS compared to those without $\mathrm{OH}(18 \pm 6 v s$. $16 \pm 5$ days, respectively, $p=0.001)$, reflecting greater medical complexity. Moreover, the actual LOS at discharge was significantly longer in $\mathrm{OH}$ patients compared to those without $\mathrm{OH}(19 \pm 12 v s$. $13 \pm 9$ days; $p=0.001)$ as was the difference between actual-predicted LOC $(1 \pm 11$ vs. $2 \pm 8 ; p=0.001)$. The change in FIM was similar in both patients with and without $\mathrm{OH}(30 \pm 26$ vs. $33 \pm 20$, respectively; $p=0.52$ ) but FIM efficiency was lower in $\mathrm{OH}$ patients $(2 \pm 2.2$ vs. $3 \pm 2.2$ without $\mathrm{OH} ; p=0.001)$. OH prolonged LOS regardless of the admission diagnosis (stroke, other neurological conditions, spinal cord dysfunction and orthopedic disorders). In conclusion, $\mathrm{OH}$ is present in a significant proportion of patients undergoing acute rehabilitation and adversely impacts the recovery of these patients as measured by lower FIM efficacy and a prolonged 
rehabilitation period to achieve the same functional gain as patients without $\mathrm{OH}$

\section{Virtual Poster \#62}

\section{Impact of patisiran, an RNAi therapeutic, on orthostatic intolerance in patients with hereditary transthyretin-mediated amyloidosis}

D.P. Judge ${ }^{1}$, A. González-Duarte ${ }^{2}$, A. Dispenzieri ${ }^{3}, \mathrm{H} \mathrm{Lin}^{4}$, M. Merkel ${ }^{4}$, Y. Wang ${ }^{4}$, M. Polydefkis ${ }^{5}$

${ }^{1}$ Medical University of South Carolina, Charleston, SC, USA;

${ }^{2}$ Instituto Nacional de Ciencias Médicas y Nutrición, Salvador Zubirán, Mexico City, Mexico; ${ }^{3}$ Mayo Clinic, Rochester, MN, USA; ${ }^{4}$ Alnylam Pharmaceuticals, Cambridge, MA, USA; ${ }^{5}$ Johns Hopkins University, Baltimore, MD, USA

Hereditary transthyretin-mediated (hATTR) amyloidosis is a rapidly progressive, multisystem disease which can impact numerous organs, including peripheral and autonomic nerves and heart. Orthostatic intolerance (OI), caused by amyloid deposition in the autonomic nervous system, is commonly reported in patients with hATTR amyloidosis. OI increases the risk that patients will experience clinically significant events, such as presyncope, syncope, and falls. In the Phase 3 APOLLO study, patisiran demonstrated improvement in OI. This analysis further describes the impact of patisiran on OI symptomatology. APOLLO was an international, randomized $(2: 1)$, double-blinded, placebo-controlled study of patisiran $0.3 \mathrm{mg} / \mathrm{kg}$ or placebo IV q3W in patients with hATTR amyloidosis with polyneuropathy (NCT01960348). COMPASS-31 is a patient-reported autonomic symptoms questionnaire containing 31-items evaluating 6 autonomic domains. In the OI domain, patients were asked, "In the past year, have you ever felt faint, dizzy, 'goofy', or had difficulty thinking soon after standing up from a sitting or lying position." Change in presence and severity of these OI symptoms collected from the OI domain of COMPASS-31 were evaluated descriptively. APOLLO enrolled 225 patients: median age 62 years, $74 \%$ male, $43 \%$ V30M, FAP Stage $1(46 \%)$ and $2(53 \%), 56 \%$ in the pre-defined cardiac subpopulation. At baseline, about two-thirds of patients reported mild to severe symptoms of OI in the past year. After 18 months, patisiran-treated patients were threefold more likely to report improvement in OI symptoms compared to placebo (30\% vs. $10 \%$, respectively). Patisiran-treated patients were also less likely to worsen on these symptoms compared to placebo-treated patients after 18 months ( $14 \%$ vs. $23 \%$, respectively). Following 18 months of treatment, patients treated with patisiran were three times more likely to improve in their OI symptoms versus their own baseline compared to placebo. These data reinforce the clinical benefit of patisiran in addressing the debilitating autonomic symptoms of hATTR amyloidosis.

Funding: Alnylam Pharmaceuticals.

\section{Virtual Poster \#63}

\section{Activating lower body muscles prior to standing mitigates symptoms of initial orthostatic hypotension}

N. Sheikh, S. Ranada, M. Lloyd, D. McCarthy, R.S. Sheldon, A. Phillips, D.V. Exner, M. Runte, S.R. Raj Department of Cardiac Sciences, Libin Cardiovascular Institute, University of Calgary, Calgary, Alberta, Canada
Background: Initial orthostatic hypotension (IOH) is a form of orthostatic intolerance that occurs in $\sim 1$ in 10 of patients with unexplained syncope. Clinical recommendations to mitigate symptoms include sitting up first or standing up slowly. Since IOH is likely due to a large muscle activation reflex with refractoriness, we hypothesized that pre-activating lower body muscles or leg crossing/ tensing immediately after standing would alleviate symptoms of IOH. Methods: After informed consent, subjects completed two sit-to-stand and one control maneuver in a randomized, cross-over study. Maneuvers included knee raises while seated (KNEE), leg crossing after standing (LEG), and no intervention (CONTROL). Beat-to-beat systolic blood pressure (SBP), stroke volume (SV), and systemic vascular resistance (SVR) were measured with a standardized device (Nexfin) and recorded for offline analyses. Participants rated their symptoms immediately upon standing using the Vanderbilt Orthostatic Symptoms Score (VOSS; range 0-90). Data are presented as mean \pm standard error of the mean (SEM).

Results: A total of 24 female IOH participants ( $32 \pm 2$ years; body mass index $24 \pm 1 \mathrm{~kg} / \mathrm{m}^{2}$ ) were enrolled. The mean reduction in SBP following KNEE $(24 \pm 4 \mathrm{mmHg})$ and LEG $(21 \pm 4 \mathrm{mmHg})$ were significantly lower versus CONTROL $(33 \pm 4 \mathrm{mmHg}), \mathrm{p}=0.016$ and $\mathrm{p}<0.001$, respectively. Symptoms were reduced with both KNEE $(9 \pm 2 ; p=0.01)$ and LEG $(9 \pm 3 ; p=0.007)$ vs. CONTROL $(13 \pm 3)$. The increase in SV at nadir SBP was significantly higher in both KNEE $\left(11 \pm 3 \mathrm{~mL} / \mathrm{m}^{2} ; \mathrm{p}=0.0006\right)$ and LEG $\left(9 \pm 4 \mathrm{~mL} / \mathrm{m}^{2}\right.$; $\mathrm{p}=0.003)$ vs. control $\left(0.3 \pm 3 \mathrm{~mL} / \mathrm{m}^{2}\right)$. The drop in SVR was not significantly altered with KNEE $\left(613 \pm 51\right.$ dynes $\left.\mathrm{s} \mathrm{cm}^{-5} ; \mathrm{p}=0.47\right)$ and LEG $\left(525 \pm 74\right.$ dynes $\left.\mathrm{s} \mathrm{cm} \mathrm{cm}^{-5} ; \mathrm{p}=0.37\right)$ vs. CONTROL $\left(572 \pm 75\right.$ dynes $\left.\mathrm{s} \mathrm{cm}^{-5}\right)$.

Conclusion: Symptoms of IOH were improved, and the drop in SBP was mitigated in both the KNEE and LEG maneuvers. These data suggest that the improvement of SBP and symptoms following the use of physical interventions are associated with an increase in SV immediately after standing.

\section{Virtual Poster \#64}

Autonomic function testing in patients with end-stage heart failure following continuous-flow left ventricular assist device implantation

M.G. Lloyd, J. Jorge, J. Angihan, D. Chew, L.Y. Lei, K. Kogut, N. Sharma, J. Howlett, N. Fine, S.R. Raj

Department of Cardiac Sciences, Libin Cardiovascular Institute of Alberta, University of Calgary, Calgary, Alberta, Canada

Background: Continuous-flow left ventricular assist devices (CFLVAD) are increasingly utilized in patients with advanced heart failure. Despite reports of orthostatic symptoms in these patients, autonomic function is not well understood. The objectives of this study were 1) to assess the feasibility of autonomic investigation, and 2) to investigate the changes in autonomic function, in patients following CF-LVAD implantation. We hypothesized that reduced autonomic function following CF-LVAD implantation would impair pressure (BP) responses to autonomic tests.

Methods: In 14 patients (all male, mean age $=56.8 \pm 12$, $7=$ HeartWare, $1=$ HeartMate II, $5=$ HeartMate III), autonomic function tests were performed before (baseline [BL]) and twice after (1 month [POST] and 3-6 months [LATE]) CF-LVAD implantation. Continuous heart rate (HR) and BP were collected (Nexfin). Patients performed a 10 -min head-up tilt to $70^{\circ}$, cold pressor test (CPT), and Valsalva maneuver. Data were analyzed with repeated-measures ANOVA, and are reported as mean \pm SE. 
Results: Resting mean arterial pressure (MAP) was not significantly different between BL $(65 \pm 2 \mathrm{mmHg})$, POST $(66 \pm 3 \mathrm{mmHg})$, or LATE $(67 \pm 3 \mathrm{mmHg}$, all $\mathrm{p}>0.8)$. The MAP response during the last minute of tilt was not significantly different between BL $(1 \pm 2 \mathrm{mmHg})$, POST $(1 \pm 2 \mathrm{mmHg})$, or LATE $(2 \pm 2 \mathrm{mmHg}$, all $\mathrm{p}>0.9)$. BP pulsatility was reduced from $\mathrm{BL}(42 \pm 2 \mathrm{mmHg})$ compared to POST $(20 \pm 3 \mathrm{mmHg}, \quad \mathrm{p}<0.0001)$ and LATE $(23 \pm 2 \mathrm{mmHg}$, both $\mathrm{p}<0.0001)$. The CPT MAP response was not significantly different between $\mathrm{BL} \quad(5 \pm 2 \mathrm{mmHg})$, POST $(6 \pm 2 \mathrm{mmHg})$, or LATE $(6 \pm 2 \mathrm{mmHg}$, all $\mathrm{p}>0.9)$. A square-wave Valsalva maneuver was present in most patients (before and after implant). Pressure recovery time was not different between BL $(23 \pm 6 \mathrm{~s})$, POST $(20 \pm 6 \mathrm{~s})$, or LATE $(21 \pm 6 \mathrm{~s}$, all $\mathrm{p}>0.8)$. Due to the high prevalence of pacemakers/defibrillators, HR responses to autonomic testing could not be evaluated.

Conclusions: Autonomic function appears to be unaffected by CFLVAD implantation, despite a marked reduction in BP pulsatility. Due to the low pulsatility, the square wave Valsalva pattern, and the presence of pacemaker control of HR in many patients, classical responses to autonomic tests are difficult to evaluate.

\section{Virtual Poster \#65}

\section{Assessing the relationship between orthostatic hypotension and cognitive impairment}

\author{
J. Baker ${ }^{1}$, Malcolm Sherwood ${ }^{2}$, Jennifer Fogarty ${ }^{1}$, Michael Borrie ${ }^{1}$, \\ Jaspreet Bhangu ${ }^{1}$ \\ ${ }^{1}$ Parkwood Institute, St. Joseph's Healthcare, London, ON, Canada; \\ ${ }^{2}$ Western University, Schulich School of Medicine, London, ON, \\ Canada
}

Background: Individuals with orthostatic hypotension $(\mathrm{OH})$ experience a significant and persistent blood pressure (BP) drop ( $\geq 20$ / $10 \mathrm{mmHg}$ ) in the upright position, which may result in cerebral hypoperfusion. There is growing evidence that $\mathrm{OH}$ and cerebral hypoperfusion contribute to cognitive decline with specific links to executive dysfunction, white matter hyperintensities and decreased attention. The objective of this study was to estimate the prevalence of $\mathrm{OH}$ in a well-characterized group of community dwelling older adults and examine links with cognitive domains.

Methods: This is a retrospective, cross-sectional analysis of a sample of participants from the Canadian Consortium of Neurodegeneration and Aging (CCNA); a national longitudinal study of community dwelling elderly people across Canada. Clinical and neurocognitive data from cognitively intact elderly $(\mathrm{CEI})(\mathrm{n}=57)$, and patients with mild cognitive impairment (MCI) $(n=31)$ and Alzheimer's disease $(\mathrm{AD})(\mathrm{n}=30)$ were extracted from the COMPASS-ND cohort study. Blood pressure was measured using a manual cuff in the supine position and after 3 min of standing.

Results: A comparison of the three cohorts showed AD patients were significantly older relatively to MCI and CIE ( $78 \pm 7$ years vs. $74 \pm 7$ years vs. $71 \pm 6$ years, respectively; $p<0.001)$, and the duration of symptoms were longer compared to MCI patients $(5.5 \pm 3$ years vs. $3.5 \pm 3$ years; $p<0.05)$. Notably, there was evidence of an increased prevalence of $\mathrm{OH}$ in participants with greater degrees of cognitive impairment (Alzheimer's disease, 23\%; mild cognitive impairment, 19\%; cognitively intact elderly, 14\%). Those with cognitive impairment showed greater degrees of frontal lobe impairment, including tests of executive dysfunction, which was significantly correlated with the orthostatic SBP drop $(\mathrm{p}=0.02)$.

Conclusion: Preliminary evidence from a longitudinal cohort of individuals with neurodegeneration establishes a link between $\mathrm{OH}$ and cognitive impairment. Furthermore, there is evidence that those with $\mathrm{OH}$ in this cohort had higher degrees of executive dysfunction. Further study is warranted to explore these links in greater detail in the larger cohort and explore possible pathophysiological mechanisms.

\section{Virtual Poster \#66}

POTS, pain, and polypharmacy: how pharmacogenetics may help

\section{S. Behl, P.R. Fischer, M. Farrell}

Department of Pediatric and Adolescent Medicine, Mayo Clinic, Rochester, MN, USA

Introduction: Youth with complex medical conditions, including dizziness, chronic fatigue, and pain, are often treated with numerous medications. Pharmacologic treatment, however, may aggravate symptoms. We reviewed our experience with pharmacogenetic testing to examine if genetic alterations in medication metabolism might account for some of the symptoms in patients taking multiple medications, and therefore provide the option of adjustments to medication choice and dosing. Methods: Our practice sees adolescents and young adults referred from around the United States with chronic debilitating symptoms, including many with concerns for postural orthostatic tachycardia syndrome (POTS). Most of these patients have seen multiple physicians and subsequently received many different treatments for their concerns. A sample of youth seen from August of 2017 to June of 2019 with complex conditions had pharmacogenetic testing done to assess for risk of altered metabolism of their drugs.

Results: A total of 83 patients underwent pharmacogenetic testing (61 [74\%] female, median age 15 [range 10-19] years, median number of medications uses at presentation 9). In 69 patients (83\%) we found at least one actionable gene-drug interaction with at least one of their medications, leading to altered metabolism of their prescriptions. Of the 48 patients on antidepressants, 13 (27\%) had variations in medication metabolism that could lead to excessive medication effects, and $14(29 \%)$ had medication metabolism variations that could lead to reduced efficacy. Of 16 patients on beta blockers, $10(63 \%)$ had reduced metabolism and could have had adverse effects from excessive blood levels. Of 30 patients on proton pump inhibitors (PPIs), 9 (30\%) had reduced metabolism, and 21 (70\%) had unusually rapid metabolism; thus all had risk of either PPI side effects or incomplete efficacy. Of 17 females who were taking ethinyl estradiol, $3(18 \%)$ were found to have Factor 5 Leiden (F5L) mutations with risk of coagulopathy. In 44 other females, 3 were found to have a thrombophilic variant in prothrombin.

Conclusion: Amongst patients with complicated symptoms, altered pharmacogenetic-based medication metabolism is common and carries implications for appropriate medication use. Testing for common gene-drug interactions may therefore be beneficial for patients.

\section{Virtual Poster \#67}

\section{Median arcuate ligament syndrome in children: a single center experience}

B. Kakavand ${ }^{1}$, R.C. Burns ${ }^{2}$, A. Centner ${ }^{3}$, A. Casas-Melley ${ }^{4}$ ${ }^{1}$ Department of Pediatric Cardiology, Nemours Children's Hospital, Orlando, FL, USA; ${ }^{2}$ Department of Surgery, Riley Children's Hospital, Indianapolis, IN, USA; ${ }^{3}$ University of Central Florida, Orlando, FL, USA; ${ }^{4}$ Department of Surgery, Nemours Children's Hospital, Orlando, FL, USA

Purpose: Data on median arcuate ligament syndrome (MALS) in children is scant. It is postulated that MALS can cause chronic 
abdominal pain. It is unclear what percentage of children with this condition are symptomatic and what comorbidities are associated with this syndrome.

Methods: In this retrospective study, data on consecutive patients in a single center diagnosed coincidentally with MALS during routine echocardiogram were reviewed. Symptom burden, comorbidities, and the effect of anthropometric indices on MALS were investigated. Descriptive statistics and nonparametric tests were used to describe the findings and to compare variables with non-Gaussian distribution. Results: Between 2013 and 2020, there were 82 children, 55 females $(67 \%)$, mean age $13.9 \pm 3.2$ years, with MALS and complete record. Mean velocity across the stenotic area was $2.6 \pm 0.4 \mathrm{~m} / \mathrm{s}$. Forty-six patients (57\%) had abdominal pain. Age, gender, weight, BMI, Doppler velocity had no statistically significant influence on symptom occurrence. Conversely, patients with joint hypermobility and symptoms of orthostatic intolerance were more likely to have abdominal pain from MALS. Of 24 patients with joint hypermobility, 18 patients had abdominal pain $(\mathrm{p}=0.027)$. Thirty-eight patients with OI with MALS complained of abdominal pain vs. 13 patients with OI and no abdominal pain $(\mathrm{p}=<0.0001)$.

Conclusion: Nearly half of patients with MALS had abdominal pain. Age, gender, weight, and the degree of stenosis had no statistically significant influence on symptom occurrence. Conversely, orthostatic intolerance in general and POTS in particular and finding of joint hypermobility on exam predicted higher propensity to abdominal pain in MALS.

\section{Virtual Poster \#68}

\section{Behavioral health factors associated with diagnosis of and prescription of medication for pediatric autonomic dysfunction}

P.R. Fischer ${ }^{1}$, S. Segner ${ }^{1}$, A. Fahrenkamp ${ }^{2}$, M. Tsai Owens ${ }^{3}$, J. Geske $^{1}$, D. Hofschulte ${ }^{1}$, M. Farrell ${ }^{1}$, K. Klaas ${ }^{1}$, C. Harbeck-Weber ${ }^{1}$, B. Biggs ${ }^{2}$

${ }^{1}$ Department of Pediatric and Adolescent Medicine and ${ }^{2}$ Department of Psychology, Mayo Clinic, Rochester, MN, USA; ${ }^{3}$ Department of Psychology, Seattle Children's Hospital, University of Washington, Seattle, WA, USA

Introduction: Some adolescents presenting with fatigue, postural dizziness, and abdominal discomfort have no evidence of identifiable structural pathology and are thought to have autonomic dysfunction, and some such patients also have postural tachycardia. Within this group of patients, it is not clear how pre-existing lifestyle factors, symptoms, emotional distress, and management relate to the presence or absence of postural tachycardia. Thus, we prospectively evaluated adolescents presenting to our referral center with symptoms suggestive of autonomic dysfunction.

Methods: We enrolled consenting patients aged 13-22 years who presented to our diagnostic clinic between September 2017 and December 2018 with symptoms suggestive of autonomic dysfunction. Data collection included lifestyle factors (fluid and salt intake, physical activity, sleep) and validated scales of autonomic symptoms, depression, anxiety, and functional disability. Patients underwent standard 70 degree head-up tilt testing for $10 \mathrm{~min}$. Clinician assigned diagnoses were recorded from the medical record. We compared findings of patients with excessive postural tachycardia and those without.

Results: Of 165 patients ( $85 \%$ female, $80 \%$ non-Hispanic white, mean age 15.7 years), 59 (33\%) had excessive postural tachycardia, and 58 $(32 \%)$ received a diagnosis of postural orthostatic tachycardia syndrome (POTS), with 91\% agreement between tilt table test result and
POTS diagnosis. Patients with excessive postural tachycardia reported greater fluid intake ( $\mathrm{p}=0.03$ ) but no difference in salt intake, level of physical activity, or sleep. The groups did not differ on autonomic symptom scores, functional disability, and level of depression and anxiety symptoms. Medications (such as beta blockers) were more likely prescribed to those with POTS.

Conclusions: Among youth seeking tertiary care with symptoms suggestive of autonomic dysfunction, youth with and without excessive postural tachycardia were quite similar in lifestyle factors, anxiety and depression symptoms, and functional disability. Patients with excessive postural tachycardia at the time of consultation had higher water intake (perhaps due to previous medical advice) and were more likely to be prescribed medications. It is not clear that excessive postural tachycardia actually differentiates between important sub-groups of the population presenting with symptoms suggestive of autonomic dysfunction. Further studies could look at presenting features as related to treatment and outcomes.

Funding: This research was supported in part by Greg and Beth Wahl.

\section{Virtual Poster \#69}

Clinical audit of clinical impact of Head-Up Tilt Test in children and young people

S. $\mathrm{Ng}^{1}$, G. Parker ${ }^{2}$, D. Moore ${ }^{3}$, W.P. Whitehouse ${ }^{4}$

${ }^{1}$ Department of Paediatrics, Nottingham Children's Hospital, Nottingham University Hospitals NHS Trust, Nottingham, UK; ${ }^{2}$ Department of Clinical Neurophysiology, Nottingham University Hospitals NHS Trust, Nottingham, UK; ${ }^{3}$ Department of Paediatric Neurology, Nottingham Children's Hospital, Nottingham University Hospitals NHS Trust, Nottingham, UK; ${ }^{4}$ School of Medicine, University of Nottingham, Nottingham, UK

Background: The Head-Up Tilt Test (HUTT) is an established test for adult patients with severe, frequent, or otherwise difficult to diagnose transient loss of consciousness (TLOC). Children have attended our hospital for HUTT over the past 20 years, and a clinical audit identified several areas for improvement which were implemented. We now report the first re-audit of the clinical impact the current HUTT procedure has, since the introduction of a comprehensive written HUTT report.

Aim: This clinical audit assessed the usefulness of the HUTT report, which should have included an explicit diagnosis and advice on treatment. It also looked at the use of the diagnosis in clinical correspondence and patients' outcomes at 12 months or more follow-up. Methods: The medical charts of 72 consecutive pediatric patients, who underwent 73/100 HUTTs between 2009 and 2019, with complete analyzable data, were reviewed. Over this period approximately $100 / 1200(8 \%)$ tertiary consults seen in "syncope" clinics were referred for HUTT. Information about indications, results, diagnosis explicitly given in HUTT report, and diagnosis used at follow-up were extracted.

Results: The patients were aged 3-18 years (median 15; IQR 11-16); $24 / 72(33 \%)$ were male. In 37/73 (51\%) HUTTs frequent or severe TLOC was the indication. $23 / 73(32 \%)$ had a positive symptomatic test, while $37 / 73(51 \%)$ had a negative asymptomatic test. $35 / 73$ (48\%) had a diagnosis explicitly mentioned in the HUTT report, the most common being vasovagal syncope in 23/73 (32\%). 44/73 (60\%) HUTTs led to specific treatment recommendations. Definite clinical improvement, clear from the medical charts, was maintained for 12 months or more after 30/73 (41\%) HUTTs. The diagnosis given in the HUTT report continued to be used at clinical follow-up after 30/35 (86\%) HUTTs. 
Conclusions: Although the yield of useful information was $49 \%$, a specific diagnosis from HUTT was only made in $32 \%$. These diagnoses were used at follow-up in $86 \%$. Overall, $41 \%$ were clinically improved a year after HUTT. Data from HUTT seems to have had a useful impact on patient management, in the selected cases.

\section{Virtual Poster \#70}

\section{Chronic transcutaneous vagal nerve stimulation in hyperadrenergic POTS patients}

D. Shiffer ${ }^{1}$, R. Furlan ${ }^{1}$, F. Barbic ${ }^{1}$, R. Zamuner ${ }^{2}$, M. Minonzio ${ }^{1}$, F. Dipaola $^{1}$, B. Cairo ${ }^{3}$, A. Porta ${ }^{3}$, S. Rigo ${ }^{1}$, V. Mundula ${ }^{1}$, E. Tobaldini ${ }^{4}$, L. Furlan ${ }^{4}$, N. Montano ${ }^{4}$, I. Biaggioni ${ }^{5}$, A. Diedrich ${ }^{5}$

${ }^{1}$ Internal Medicine, Humanitas Clinical and Research Center-IRCCS, Humanitas University, Rozzano, Italy; ${ }^{2}$ Departamento de Kinesiología, Universidad Católica del Maule, Maule, Chile;

${ }^{3}$ Department of Biomedical Sciences for Health, University of Milan, Milan, Italy; ${ }^{4}$ Ospedale Policlinico IRCCS, University of Milan,

Milan, Italy; ${ }^{5}$ Vanderbilt Autonomic Dysfunction Center, Vanderbilt University, Nashville, TN, USA

Background: In postural tachycardia syndrome (POTS) many symptoms and signs may be related to sympathetic over-activity. An increase of cardiac vagal modulation with a concomitant decrease of cardiac sympathetic drive may result in clinical improvement of POTS. Transcutaneous vagal nerve stimulation (tVNS) proved to be effective in increasing cardiac vagal modulation.

Aim: To evaluate whether chronic tVNS might reduce orthostatic symptoms and change hemodynamic parameters during orthostasis in POTS.

Methods: 11 POTS patients ( $38 \pm 4$ years; BMI $22.5 \pm 1.4 \mathrm{~kg} / \mathrm{m}^{2}$ ) with possible hyperadrenergic phenotype [upright: norepinephrine $>600 \mathrm{pg} / \mathrm{ml}$ ] underwent continuous ECG, BP and respiratory activity recordings in supine position and during a $75^{\circ}$ head-up tilt before (baseline) and after chronic tVNS. Electrical stimulation was delivered through the skin at the right ear concha (pulse width $200 \mu \mathrm{s}$; frequency $25 \mathrm{~Hz}$ ) for $4 \mathrm{~h}$ daily for two weeks with a noninvasive, battery-powered device (NEMOS; Cerbomed, Germany). Stimulation amplitude was individually adjusted to maximal tolerable level $(2.1 \pm 0.6 \mathrm{~mA}$, mean $\pm \mathrm{SD})$. A modified Vanderbilt Orthostatic Symptoms Scale was used to quantify symptoms (mental clouding, blurred vision, short of breath, rapid heartbeat, tremulousness, chest discomfort, headache, lightheaded, nausea) in a range of zero (no symptom) to 10 (maximum intensity). Power spectrum analysis of heart rate (HR) variability provided the low frequency (LF) and high frequency (HF) power and LF/HF ratio as an index of the cardiac sympatho-vagal instantaneous interaction.

Results: After chronic tVNS, tilt induced a lower increase in HR $(\Delta 36.1 \pm 8.6$ from $86.1 \pm 5.1 \mathrm{~b} / \mathrm{min}$ vs. $\Delta 40.0 \pm 3.6$ from $81.1 \pm 3.8 \mathrm{~b} / \mathrm{min}$, respectively) and a significant $(\mathrm{p}<0.04)$ lower increase of $\mathrm{LF} / \mathrm{HF}(\Delta 10.3 \pm 1.7$ from $1.7 \pm 0.3)$ compared to baseline $(\Delta 21.2 \pm 4.5$ from $3.8 \pm 0.7)$. Upright $\mathrm{BP}$ and respiration remained unchanged. Chronic tVNS resulted in a significant reduction in overall symptoms intensity $(4.1 \pm 2.2$ vs. $5.8 \pm 2.1 ; \mathrm{p}<0.05$, respectively), in perception of rapid heartbeat (5.2 \pm 2.6 vs. $7.4 \pm 2.6 ; \mathrm{p}<0.05$, respectively) and chest discomfort $(3.9 \pm 2.8$ vs. $6.3 \pm 2.6 ; \mathrm{p}<0.05$, respectively) compared to baseline.

Conclusion: In hyperadrenergic POTS patients, chronic tVNS improved overall orthostatic symptoms and specifically alleviated symptoms of rapid heartbeat and chest discomfort. Chronic tVNS reduced the orthostatic increase in LF/HF ratio suggesting an enhanced cardiac vagal activity after tVNS. Additionally, there was a trend of lower HR increase during the orthostatic stress, although not significant.

Funding: The study was supported by a grant from Dysautonomia International, IRB-ID 986.

\section{Virtual Poster \#71}

\section{Perception of cognitive impairment in individuals with POTS}

E.M. Rich, C. Hollingsworth, V. Boyette, A. Vas

School of Occupational Therapy, Texas Woman's University, Dallas, TX, USA

Cognitive impairment is a common but poorly understood symptom in postural tachycardia syndrome (POTS). Despite the use of numerous pharmacologic and non-pharmacologic therapeutic options for managing physical manifestations of symptoms, cognitive deficits frequently persist. At this time, the literature is limited, with no studies documenting treatment interventions. As a result of cognitive deficits, patients commonly report difficulty with word-finding, processing speed, focused attention, memory, and executive functioning skills; these impairments may lead to functional deficits in the areas of social participation, driving, work, and education to name a few. Clinicians frequently hear complaints of these cognitive deficits but may have a limited appreciation of the breadth of impact and lack of treatment options to offer patients. In a large $(\mathrm{n}=958)$ survey study of individuals $18-60$ years of age $[\mathrm{M}=32.63 \pm 10.52], 93.4 \%$ of participants reported "thinking challenges" within the week prior. The survey included both quantifiable and free response items which allowed for rich data inquiry. Coding and analysis of free-response data describing self-reported "thinking challenges" led to development of themes and frequently cited words. Identified themes included memory, attention, executive function, information processing, psychosocial, brain fog, and physical symptoms. The most frequently utilized verbiage and frequency of occurrence included "word(s)" (as in word-finding) [1215], "forget" [787], "feel" [692], "think" [643], "brain" [604], "timing" [576], "remember" [439], and "fog" (as in brain fog) [404]. These findings are consistent with previous research surrounding subjective and objective assessments of cognitive impairment in POTS patients. Participant quotes in the study highlight exacerbating and relieving factors; emotional impact; and functional deficits. This data provides insight into the patient experience in order to better understand needs for assessment and treatment intervention in addressing cognitive dysfunction in POTS. Additional research is indicated to determine the efficacy of cognitive interventions in order to increase independence, functional participation, and quality of life.

\section{Virtual Poster \#72}

\section{Screening for novel autoantibodies in POTS}

S. Vernino ${ }^{1}$, M. Bryarly ${ }^{1}$, R. Schubert ${ }^{2}$, M.R. Wilson ${ }^{2}$

${ }^{1}$ Department of Neurology and Neurotherapeutics, UT Southwestern Medical Center, Dallas, TX, USA; ${ }^{2}$ University of California San Francisco Weill Institute for Neurosciences, San Francisco, CA, USA

Background: Postural tachycardia syndrome (POTS) is characterized by excessive increase in heart rate when standing. The precise pathophysiology of POTS remains unclear. Association with various autoimmune disorders has been reported. Several autoantibodies have been proposed to be important in POTS including antibodies against G-protein coupled receptors (GPCRs) like the alphal adrenergic 
receptor and muscarinic acetylcholine receptors. The reliable detection of such antibodies has been challenging. An evolving powerful method for novel autoantibody detection utilizes next generation sequencing methods to display peptide antigens from the entire human genome in a phage display. This phage display library can be used to identify autoantibodies in serum or CSF samples in an agnostic way (i.e., without predetermination of possible antigen targets).

Methods: Clinical information and serum samples were collected from POTS patients $(n=109)$ and healthy controls $(\mathrm{N}=13)$ during the 2016 Dysautonomia International patient conference. Deidentified serum samples were incubated with a human genome phage display (20,000 genes) and bound antigens were identified by DNA sequencing. The results were analyzed for the whole genome results, and GPCR genes were examined separately.

Results: Adjusting for multiple comparisons, there were no antigens that showed statistically significant binding by serum antibodies in POTS patients as a group compared to controls. Individual patient sera showed binding to individual antigens including various GPCR. Further analysis and clinical correlations are ongoing.

Conclusions: An agnostic phage display method can be used to detect autoantibodies. This method may be useful to identify novel autoantibodies or to confirm putative antibody targets in POTS. No consistent autoantibody was strongly associated with POTS in this pilot group but additional analysis of individual subject results with clinical correlation and comparison to other antibody detection methods will be important.

Funding: Dysautonomia International.

\section{Virtual Poster \#73}

\section{Work ability assessment in active workers with postural tachycardia syndrome}

S. Cavalieri ${ }^{1}$, M. Minonzio ${ }^{2}$, F. Dipaola ${ }^{2,3}$, D. Shiffer ${ }^{2}$, B. Cairo ${ }^{4}$, R.A. Zamuner ${ }^{5}$, I. Capitanelli ${ }^{1}$, N. Magnavita ${ }^{1,6}$, R. Furlan ${ }^{2,3}$, F. Barbic $^{2,3}$

${ }^{1}$ Department of Life Sciences \& Public Health, Università Cattolica del Sacro Cuore, Rome, Italy; ${ }^{2}$ Internal Medicine, Humanitas Clinical and Research Center- IRCCS, Rozzano, Milan, Italy; ${ }^{3}$ Humanitas University, Department of Biomedical Sciences, Pieve Emanuele, Milan, Italy; ${ }^{4}$ Department of Biomedical Sciences for Health, University of Milan, Milan, Italy; ${ }^{5}$ Departamento de Kinesiología, Universidad Católica del Maule, Maule, Chile; ${ }^{6}$ Department of Woman, Children \& Public Health, Fondazione Policlinico A. Gemelli-IRCCS, Rome, Italy

Background: It is well known that postural tachycardia syndrome (POTS) negatively impacts patient's quality of life. However, little is known about the consequences of the disease on work life and performance. Working activity may prevent chronic disability by maintaining patients physically and mentally active.

Aims: To evaluate the work ability and the related comorbidities in a group of POTS patients consecutively enrolled by the Cardiovascular Autonomic Disorders Unit of Humanitas Research Hospital.

Methods: 22 POTS patients regularly engaged in working activity (POTS) (20 F, age $36 \pm 12$ years, BMI $21.3 \pm 3.1 \mathrm{~kg} / \mathrm{m}^{2}$, BP $110 \pm 10 / 65 \pm 7 \mathrm{mmHg}$, HR increase during standing $31 \pm 12$ beats/min) and 18 sex and aged-matched controls (Ctrl) with similar working activity underwent a clinical evaluation and filled out the Work Ability Index questionnaire (WAI). This is a validation tool that furnishes a self-assessment of employees' work ability. The index is determined based on the answers to a series of questions that take into consideration the demands of work, the worker's health status and resources. The physician in charge rated the responses according to the seven items (current WA compared to the life-time best, WA in relation to the job demands, currents diseases diagnosed, estimated work impairment and sick leave in the last year due to the diseases, own prognosis of WA 2 years from now, mental resources; score range 7-49). He finally assigns patients to 4 categories based on their ability to work: poor (7-27), moderate (28-36), good (37-43), excellent (44-49). The WAI also provides a quantitative evaluation of comorbidities.

Results: Working capacity was significantly reduced in POTS compared to $\mathrm{Ctrl}(29.8 \pm 7$ vs. $44.7 \pm 5 \mathrm{p}<0.01)$. POTS were also characterized by more comorbidities than Ctrl $(8 \pm 5$ vs. $1 \pm 1$ $\mathrm{p}<0.001)$ particularly involving the musculoskeletal $(77 \%)$ and gastrointestinal $(60 \%)$ system, headache, anxiety-depression and sleep disorders (50\%). Hypermobile Ehlers-Danlos syndrome and fibromyalgia were present in $23 \%$ and $18 \%$ of POTS, respectively. Conclusion: The WAI and all its items showed a reduced working ability of POTS and identified comorbidities that may promote frailty at work. A holistic approach may promote therapeutic and preventive intervention aimed to keep these patients active.

Funding: Supported by the Italian Ministry of Health, grant \# RF2013-02355242.

\section{Virtual Poster \#74}

Relationship between work ability and cardiac autonomic profile in postural tachycardia syndrome

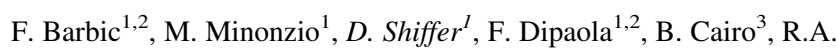
Zamuner $^{4}$, S. Cavalieri ${ }^{5}$, I. Capitanelli ${ }^{5}$, N. Magnavita ${ }^{5,6}$, R. Furlan ${ }^{1,2}$ ${ }^{1}$ Internal Medicine, Humanitas Clinical and Research Center-IRCCS, Rozzano, Milan, Italy; ${ }^{2}$ Humanitas University, Department of Biomedical Sciences, Pieve Emanuele, Milan, Italy; ${ }^{3}$ Department of Biomedical Sciences for Health, University of Milan, Milan, Italy; ${ }^{4}$ Departamento de Kinesiología, Universidad Católica del Maule, Maule, Chile; ${ }^{5}$ Department of Life Sciences \& Public Health, Università Cattolica del Sacro Cuore, Rome, Italy; ${ }^{6}$ Department of Woman, Children \& Public Health, Fondazione Policlinico A. Gemelli-IRCCS, Rome, Italy

Background: The excessive increase of cardiac sympathetic activity during standing is a common characteristic of patients with postural tachycardia syndrome (POTS) and leads most of the symptoms and signs of orthostatic intolerance. Among them, mental clouding, blurred vision, shortness of breath, palpitation, chest discomfort, headache, lightheadedness may negatively impact on work performance. Little is known about the relationship between cardiac autonomic profile and work performance in POTS.

Aim: The aim of the study was to evaluate the relationship between cardiac autonomic response to gravitational stimulus and work ability in patients with POTS.

Methods. 19 POTS patients regularly engaged in working activity (18 $\mathrm{F}$, age $35 \pm 11$ years; BMI $21.3 \pm 3.3 \mathrm{~kg} / \mathrm{m}^{2}$ ) underwent clinical evaluation, continuous ECG, BP and respiratory activity recordings in the supine position and during a $75^{\circ}$ head-up tilt (HUT). Power spectrum analysis provided the index of cardiac sympatho-vagal relationship (LF/HF). At enrollment all POTS patients filled out the Work Ability Index questionnaire (WAI). This questionnaire is a validated tool used to make a self-assessment of employees work ability taking into consideration the role of comorbidities, by providing an individual synthetic score (range 7-49) overall corresponding to 4 categories of different work ability levels: poor (7-27), moderate (28-36), good (37-43), excellent (44-49). The 
linear regression between the individual WAI score and the increase of heart rate (HR) as well as LF/HF during HUT were also assessed. Results: During HUT, POTS patients showed an increase in HR $(\Delta$ $24.3 \pm 11.5$ from $76 \pm 12$ beats $/ \mathrm{min})$ and $\operatorname{LF} / \mathrm{HF}(\Delta 8.3 \pm 11.3$ from $3.5 \pm 3.7)$, while BP remained unchanged. A significant inverse correlation was found between individual WAI score and HUT increase of $\mathrm{HR}(\mathrm{r}-0.99 ; \mathrm{CI}-1.7$ to $-0.36 ; \mathrm{p}=0.005)$ and $\mathrm{LF} / \mathrm{HF}$ ( $\mathrm{r}-0.99 ; \mathrm{CI}-1.66$ to $-0.34 ; \mathrm{p}=0.005)$.

Conclusion: In this group of active workers with POTS the WAI score resulted strictly correlated to the intensity of cardiac sympathetic activation induced by gravitational stimulus. Indeed, the higher the increase of both HR and cardiac sympatho-vagal modulation index, the lower the WAI. These results suggest that the sympathetic overactivity may play a negative role on work capability and performance. Funding: Supported by the Italian Ministry of Health, grant \# RF2013-02355242.

\section{Virtual Poster \#75}

\section{Co-occurrence of POTS and myasthenia gravis}

\section{R. Shaik, D. Sinn, S. Jaradeh, S. Muppidi}

Department of Neurology, Stanford University, Palo Alto, CA, USA

Introduction: The incidence of postural tachycardia syndrome (POTS) and myasthenia gravis (MG) is higher in young women. While MG is a well-known antibody-mediated disease, autoimmune etiology has been proposed as one of the mechanisms in POTS.

Objective: To assess the co-occurrence of POTS and myasthenia gravis in patients at a single center.

Methods: We reviewed cases at our center with a diagnosis of both POTS and MG from January 2017 to date. We only included POTS cases confirmed with autonomic testing including tilt table study. We reviewed treatments used for POTS and overall improvement. For MG, we reviewed the antibody status and basis for MG diagnosis if seronegative. We reviewed additional information including CT chest, thymectomy status, current treatment for MG including pyridostigmine, immunosuppressive agents, and overall ongoing Myasthenia Gravis Foundation of America clinical status.

Results: We found 4 patients who had both MG and POTS. In 3 patients, symptoms suggestive of POTS and MG had a near-simultaneous onset. Mean age was 30 (range 21-39) and all were female. All patients had greater than 40 beats increase in HR during head-up tilt table without evidence of orthostatic hypotension. Three out of 4 patients had QSART testing. Only one patient had mild QSART abnormalities and was normal in 2 patients. One patient responded to B blockers. Two patients were intolerant to B blockers and improved with Ivabradine. One patient was stable on increased salt, fluid, and exercise regimen, but was on prednisone and Eculizumab for MG. Ganglionic AChR antibodies were not found in any of the patients. From a myasthenia point of view, 2 were AChR antibody-positive and two were seronegative. One patient with AChR positive status also had voltage-gated calcium antibody. Thymoma was not present in any of the 4 patients. Only one patient had thymectomy. Three patients were on oral prednisone therapy for MG. Two patients were on also on Azathioprine and intravenous immunoglobulin therapy for ongoing MG therapy.

Conclusions: POTS and MG association seems relatively rare in our clinic population. Treatment of POTS was complicated by intolerance to $\mathrm{B}$ blockers in two patients.

\section{Virtual Poster \#76}

\section{Immunogenetic risk markers in postural orthostatic tachycardia} syndrome

Z. Orban $^{1}$, A. Miller ${ }^{2}$, K. Bourne ${ }^{3}$, J. Nitis ${ }^{4}$, W. Hu ${ }^{5}$, R.A. Reinsel ${ }^{6}$, L.E. Stiles ${ }^{6,7}$, A. Fedorowski ${ }^{8}$, J. Axelsson ${ }^{8,9}$

${ }^{1}$ Department of Neurology, Northwestern University, Chicago, IL, USA; ${ }^{2}$ Department of Neural and Behavioral Sciences, Penn State Health, Hershey, PA, USA; ${ }^{3}$ Cumming School of Medicine,

University of Calgary, Calgary, AB, Canada; ${ }^{4}$ Vagelos College of Physicians and Surgeons, Columbia University, New York, NY, USA; ${ }^{5}$ Department of Radiology, Lenox Hill Hospital Northwell Health, New York, NY, USA; ${ }^{6}$ Department of Neurology, Stony Brook University, Stony Brook, NY, USA; ${ }^{7}$ Dysautonomia International, East Moriches, NY, USA; ${ }^{8}$ Department of Clinical Sciences, Lund University and Department of Cardiology, Skåne University Hospital, Malmö, Sweden; ${ }^{9}$ Department of Clinical Immunology, Karolinska University Hospital, Stockholm, Sweden

Background: Postural tachycardia syndrome (POTS) is a condition of unknown etiology characterized by chronic orthostatic intolerance and tachycardia while upright in the absence of orthostatic hypotension. Prior research suggests autoimmune involvement in POTS, with one study identifying increased risk associated with human leukocyte antigen (HLA) types in South Korean POTS patients. We sought to replicate these findings in a US population.

Methods: With IRB approval, we recruited 103 POTS participants during the Dysautonomia International 2019 patient conference. Orthostatic vitals, a detailed medical history, functional and quality of life scores, and a physical exam were obtained from participants (median age 28 years, range 13-74). Blood and urine specimens were also collected, with leukocyte DNA isolated from whole blood. Intermediate-resolution HLA typing was performed using commercially available kits with sequence specific primers (SSP-Olerup, CareDX, PA, USA) for loci A, -B, -DQA1, -DQB1, and -DRB1. Patient allele frequencies were compared to healthy Americans as reported in the Allele Frequency Net Database (AFND, Nucleic Acids Research, 2020).

Results: HLA-typed participants were primarily female (93\%), Caucasian (95\%) and non-Hispanic (88\%). Compared to population controls, POTS participants were more likely (OR 95\% CI; \% of subjects with allele) to present class I alleles $\mathrm{A} * 02: 01$ (1.37, $1.01-1.86,57 \%), \mathrm{B} * 15: 01(2.52,1.66-3.81,24 \%), \mathrm{B} * 18: 01$ (2.03, $1.22-3.39,15 \%)$, and $\mathrm{B}^{*} 40: 01(2.91,1.93-4.41,27 \%)$. Similarly, class II alleles DQA1*02:01 (1.94, 1.32-2.86, 36\%), DQB1*02:01 $(2.84, \quad 1.97-4.10,49 \%), \quad$ DRB1*03:01 (1.85, 1.30-2.65, 34\%), DRB1*08:01 (2.39, 1.26-4.5, 10\%), and DRB1*13:01 (1.80, $1.11-2.92,17 \%)$ were more common than expected in this group. Conclusions: Certain class I and II HLA genotypes are more common than expected in POTS participants, suggesting potential immunogenetic predispositions for developing POTS. Of these, DQB1*02:01 and DRB1*03:01 are established risk factors for several autoimmune diseases. Associated alleles in US POTS subjects differed from the South Korean POTS cohort, which is consistent with reports of distinct HLA associations for the same disease in ethnically disparate populations. Our data suggest that people with POTS have increased genetic risk for autoimmunity. Further exploration of the potential role of autoimmunity in POTS is warranted.

Funding: Dysautonomia International. 


\section{Virtual Poster \#77}

\section{Menstrual irregularities in pediatric patients with postural tachycardia syndrome and chronic orthostatic intolerance}

\section{Riordan ${ }^{1}$, K. Leopold ${ }^{2}$, A. Chattha ${ }^{2}$, E. Bolen ${ }^{3}$, W. Bunn ${ }^{3}$, S.} Hasan $^{3}$, K. Klaas ${ }^{2}$

${ }^{1}$ Mayo Clinic Alix School of Medicine, Mayo Clinic, Rochester, MN, USA; ${ }^{2}$ Department of Pediatrics, Mayo Clinic, Rochester, MN, USA; ${ }^{3}$ Mayo Clinic Alix School of Medicine, Mayo Clinic, Scottsdale, AZ, USA

Background: Postural tachycardia syndrome (POTS) and chronic orthostatic intolerance (OI) have been associated with multiple comorbid disorders, including anecdotal reports of menstrual disorders which may impact the severity of orthostatic symptoms. However, little evidence exists regarding concomitant menstrual complaints or strategies for menstrual management.

Methods: To better understand the intersection between dysautonomia, dysmenorrhea, and use of hormonal management, a retrospective cohort study of female pediatric patients seen at the Mayo Clinic in 2016 was conducted. The aim of this study was to explore the prevalence of menstrual disorders and dysfunction among pediatric patients with POTS and OI. Our review included all patients diagnosed with POTS or chronic OI, as well as all healthy patients seen at Mayo Clinic over the same timeframe. The results were analyzed using Chi Square test of independence, Fisher exact test, and one-way Analysis of Variance.

Results: Our review identified 185 patients diagnosed with POTS (age 12.1-18.6, mean 16.1), 202 with chronic OI (age 12.2-18.2, mean 15.9), and 928 (age 12.0-18.0, mean 15.0) healthy controls. Among patients with POTS, $81 \%$ reported at least one menstrual abnormality ("irregular period", "vaginal discharge", "abnormal flow", "cramps", and "other"), compared to $80 \%$ in the OI group ( $\mathrm{p}=0.80$ ) and $54 \%$ in the control group ( $<<0.0001)$. There was no difference in prescription of hormonal birth control between POTS (41\%) and OI $(34 \%)$ patients $(\mathrm{p}=0.14)$, though both were more likely to be prescribed hormonal contraceptives than controls $(\mathrm{p}<0.0001)$. There was no difference in average age of menarche $(\mathrm{p}=0.35)$.

Conclusion: Adolescent patients diagnosed with POTS and orthostatic intolerance report more menstrual irregularities than their healthy peers, and more often have a prescription for hormonal birth control. The incidence of menstrual abnormalities was not significantly different between patients with POTS and OI who did not meet criteria for excessive postural tachycardia. Providers caring for these patients should consider taking a thorough menstrual history to identify menstrual irregularities and adequately address the concerns of this population.

\section{Virtual Poster \#78}

\section{Subcutaneous immunoglobulin in patients with postural tachycardia syndrome}

\section{K. Kesterson ${ }^{1}$, S. Blitshteyn ${ }^{2}$ \\ ${ }^{1}$ Department of Psychiatry, University of Central Florida College of Medicine, Orlando, FL, USA; ${ }^{2}$ Department of Neurology, University at Buffalo Jacobs School of Medicine and Biomedical Sciences, Buffalo, NY, USA}

Objective: Intravenous immunoglobulin (IVIG) as an effective treatment of dysautonomia has been described in isolated case reports and one case series. We report 3 patients with postural tachycardia syndrome (POTS) refractory to standard treatment who experienced significant improvement following subcutaneous immunoglobulin (SQIG) therapy.

Methods and Results: All 3 patients (females, age 28-30) had POTS confirmed by a tilt table test, $2 / 3$ had small fiber neuropathy confirmed by quantitative sudomotor axon reflex test, and $2 / 3$ had hypermobile Ehlers-Danlos syndrome. Two patients had positive nicotinic ganglionic acetylcholine receptor antibodies at low titers, and one patient had elevated adrenergic, muscarinic, angiotensin II type I and endothelin I receptor antibodies as part of the autoimmune panel performed by CellTrend $\mathrm{GmbH}$ (Luckenwalde, Germany). None had positive anti-nuclear antibodies, anti-thyroid peroxidase antibodies or co-morbid autoimmune disorders. Six to nine months after initiation of SQIG, all three patients had improved COMPASS31 scores by $50-68 \%$ and functional ability scores two to fourfold from the pre-treatment scores. Two patients were able to either discontinue or reduce oral medications taken for POTS, and two patients were able to resume and maintain full-time employment. There were no aseptic meningitis or other significant adverse events reported.

Conclusion: SQIG may be effective in patients with POTS refractory to standard therapy and needs to be evaluated in randomized placebocontrolled trials. SQIG, which is self-administered, eliminates the need for intravenous access and nursing care and may present a safer, cheaper and more accessible therapeutic option than IVIG.

\section{Virtual Poster \#79}

The utilization of salt and electrolytes in the treatment of postural tachycardia syndrome

J.E. Hall ${ }^{1}$, K. Bourne ${ }^{1}$, L.E. Stiles ${ }^{2,3}$, R.S. Sheldon ${ }^{1}$, C.A. Shibao ${ }^{4}$, L.E. Okamoto ${ }^{4}$, E.M. Garland ${ }^{4}$, A. Peltier ${ }^{4}$, A. Diedrich ${ }^{4}$, I. Biaggioni $^{4}$, A. Gamboa ${ }^{4}$, S.R. Raj ${ }^{1,4}$

${ }^{1}$ Department of Cardiac Sciences, Cumming School of Medicine, University of Calgary, Calgary, AB, Canada; ${ }^{2}$ Dysautonomia International, East Moriches, NY, USA; ${ }^{3}$ Department of Neurology, Stony Brook University, Stony Brook, NY, USA; ${ }^{4}$ Autonomic Dysfunction Center, Department of Medicine, Vanderbilt University Medical Center, Nashville, TN, USA

Background: Postural tachycardia syndrome (POTS) is a common form of orthostatic intolerance primarily affecting female patients of child-bearing age. There are no medications FDA-approved to treat POTS and non-pharmacological treatments are frequently recommended. We sought to explore dietary salt and salt/electrolyte supplement use in POTS patients.

Methods: Data were collected using the "Diagnosis and Impact of POTS" survey. This is an IRB approved, patient community-based online survey conducted between Jul 2015-Dec 2019. It included questions relating to non-pharmacological treatment use and effects. Statistical analyses were conducted using Chi square (categorical data) and Mann-Whitney $U$ (continuous data [mean \pm SD]) tests.

Results: Of 7835 respondents with physician-diagnosed POTS, $93.6 \%$ were female $(\mathrm{n}=7336)$ and $6.4 \%$ were male $(\mathrm{n}=499)$. A total of 6870 participants disclosed their age $(88 \% \geq 18$ years). Respondents were highly symptomatic, with frequent lightheadedness (99\%), presyncope (94\%), and palpitations $(88 \%)$ within the prior 3 months. The majority of respondents $(91 \%)$ increased their dietary salt intake after POTS diagnosis, $11 \%$ saying this improved their symptoms "a lot" and 55\% "a little". The majority of respondents used a combination of dietary salt with salt/electrolyte supplements $(59 \%)$, followed by dietary salt only (33\%), and salt/electrolyte supplements only (2\%). Respondents whose symptoms improved in response to dietary salt were currently using salt/electrolyte supplements more commonly (57\%) than those whose symptoms did not improve with 
dietary salt intake $(39 \% ; \mathrm{p}<0.001)$. Interestingly, both salt/electrolyte supplement users and non-users experienced frequent lightheadedness $(99 \%, \mathrm{p}=0.2)$, and palpitations (88\% in each group, $\mathrm{p}=0.9)$ in the three months prior to survey completion. Pre-syncope was more common among salt/electrolyte supplement users (95\% vs. $93 \%, \mathrm{p}<0.001)$. Salt/electrolyte supplement users, regardless of dietary salt use, had lower scores on the RAND36 physical health composite score $(34 \pm 31$ vs. $37 \pm 33$; p < 0.001). Although there was no difference in salt/electrolyte supplement use between females and males ( $46 \%$ vs. $43 \%, \mathrm{p}=0.2$ ), use was more common in patients $<18$ years than $18+$ years $(56 \%$ vs. $45 \%$; p $<0.001)$.

Conclusion: The majority of POTS patients increased dietary salt in combination with a salt/electrolyte supplement. Factors including response to dietary salt, age, and quality of life may influence their decisions to augment treatment with salt/electrolyte supplementation. Funding: This work was supported in part by the National Center for Advancing Translational Sciences Award UL1 TR000445, and by Dysautonomia International.

\section{Virtual Poster \#80}

A comparison of health-related quality of life in autonomic disorders: postural tachycardia syndrome vs. vasovagal syncope

J.E. Hall ${ }^{1}$, J. $\mathrm{Ng}^{1}$, K. Bourne ${ }^{1}$, R.S. Sheldon ${ }^{1}$, M. Bryarly ${ }^{2}$, S. Vernino $^{2}$, A. Gamboa ${ }^{3}$, S.R. Raj ${ }^{1,3}$

${ }^{1}$ Department of Cardiac Sciences, Cumming School of Medicine, University of Calgary, Calgary, AB, Canada; ${ }^{2}$ Department of Neurology and Neurotherapeutics, UT Southwestern Medical Center, Dallas, TX, USA; ${ }^{3}$ Autonomic Dysfunction Center, Department of Medicine, Vanderbilt University Medical Center, Nashville, TN, USA

Background: Postural tachycardia syndrome (POTS) and vasovagal syncope (VVS) are two common orthostatic intolerance disorders with reduced health-related quality of life (HRQoL) compared to healthy populations. The purpose of this study is to compare HRQoL between patients with POTS and those with VVS.

Methods: The RAND 36-Item Health Survey (RAND36), a generic and coherent HRQoL survey, was completed by patients ( $>18$ years) with POTS at Dysautonomia International Patient Conferences and by VVS patients enrolled in the second Prevention of Syncope Trial. Data are reported as mean $\pm \mathrm{SD}$. Comparisons were performed using Student's t-tests.

Results: This analysis includes 176 POTS patients $(31 \pm 11$ years, $92.6 \%$ female) and 72 VVS patients ( $34 \pm 14$ years, $66.7 \%$ female). POTS patients reported decreased physical functioning (42 \pm 23 vs. $77 \pm 24 ; \mathrm{p}<0.001)$, social functioning $(44 \pm 24$ vs. $71 \pm 24$; $\mathrm{p}<0.001)$ and general health $(31 \pm 20$ vs. $60 \pm 22)$ RAND36 domain scores compared to VVS patients. POTS patients also had lower scores in the role limitations due to physical health $(11 \pm 24$ vs. $33 \pm 42 ; \mathrm{p}<0.001)$, energy and fatigue $(28 \pm 18$ vs. $51 \pm 22$; $\mathrm{p}<0.001)$, and pain $(47 \pm 25$ vs. $68 \pm 25 ; \mathrm{p}<0.001)$ domains. Role limitations due to emotional health scores $(66 \pm 41$ vs. $56 \pm 44 ; p=0.08)$, and emotional well-being ( $67 \pm 18$ vs. $69 \pm 21$; $\mathrm{p}=0.5$ ) were not different between POTS and VVS patients. Composite scores for physical health $(37 \pm 20$ vs. $69 \pm 28$; p $<0.001)$, mental health $(62 \pm 20$ vs. $77 \pm 26 ; \mathrm{p}<0.001)$ and general health $(57 \pm 19$ vs. $82 \pm 29 ; \mathrm{p}<0.001)$ were lower in POTS patients compared to VVS patients.

Conclusion: POTS patients have a reduced HRQoL when it comes to physical functioning and health compared to VVS patients, however, emotional health domains were not different. It is possible that the significant symptom burden and presence of co-morbid disorders commonly associated with POTS may contribute to poorer physical health-related quality of life in this patient population. Both groups may benefit from interventions targeted at improving physical and emotional functioning.

Funding: This work was supported in part by the National Center for Advancing Translational Sciences Award UL1 TR000445, by Dysautonomia International, and by the Canadian Institute of Health Research (CIHR).

\section{Virtual Poster \#81}

\section{Retrospective review of subjective responses to carbidopa in autonomic disorders}

\section{C.J. Park, E.P. Golden, S. Vernino}

Department of Neurology and Neurotherapeutics, University of Texas Southwestern Medical Center, Dallas, TX, USA

Background: Carbidopa inhibits L-aromatic-amino-acid decarboxylase. Due to its potential to reduce peripheral catecholamine levels, carbidopa is being explored as a novel therapy for hyperadrenergic symptoms of autonomic disorders.

Objective: This study aimed to characterize patients in the UT Southwestern autonomic disorder clinic who were prescribed carbidopa for open label treatment of autonomic symptoms and to evaluate subjective responses to therapy.

Methods: Adult patients prescribed carbidopa for autonomic symptoms from 2017 to 2019 were retrospectively identified. Participants completed a telephone interview about their subjective experience with carbidopa, and additional information was collected from medical record review.

Results: 27 eligible patients were identified. 18 patients were interviewed, and a total of 23 charts were reviewed (20 women, age range 19-71 years). Approximately half of the patients were diagnosed with POTS, one with baroreflex dysfunction, and 9 had other various symptoms of dysautonomia. Reported comorbidities were common, including migraine, gastroparesis, Ehlers-Danlos syndrome, and mast cell activation syndrome. Those who had documented plasma catecholamines had elevated standing norepinephrine (mean $1082.8 \mathrm{pg} /$ $\mathrm{ml}$ ). 17 took carbidopa (prescribed total daily dose of $75-150 \mathrm{mg}$ ), and 10 continued it for longer than 3 months. 9 patients reported better symptom control with carbidopa; one patient worsened, and the remainder felt no difference. The most commonly improved symptom was tremor; others included dizziness, tachycardia, nausea/vomiting, and sweating. 4 patients reported side effects from carbidopa. The most common reason for discontinuation of carbidopa was lack of benefit; other factors cited included cost, side effects, and improvement of symptoms.

Conclusions: In this small retrospective study, carbidopa was well tolerated in patients with dysautonomia, and half reported symptomatic benefit. Larger, placebo-controlled trials are warranted for further investigation of this therapy.

\section{Virtual Poster \#82}

Time course of autonomic symptoms in postural tachycardia syndrome (POTS) patients: 2-year follow-up results

F. Dipaola ${ }^{1,2}$, C. Barberi ${ }^{2}$, E. Castelnuovo ${ }^{2}$, M. Minonzio ${ }^{1}, D$.

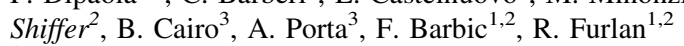

${ }^{1}$ Internal Medicine, Humanitas Clinical and Research Center-IRCCS, Rozzano, Milan, Italy; ${ }^{2}$ Department of Biomedical Sciences, 
Humanitas University, Pieve Emanuele, Milan, Italy; ${ }^{3}$ Department of Biomedical Sciences for Health, University of Milan, Milan, Italy

Background: Postural tachycardia syndrome (POTS) is a multifactorial condition capable of chronically reducing the quality of life and the work ability of patients. The aim of the study was to assess the burden of autonomic symptoms in a cohort of POTS patients over a 2-year follow-up.

Methods: Patients' clinical profile was assessed by the 31-item Composite Autonomic Symptom Score (COMPASS 31) evaluating six autonomic function domains (orthostatic intolerance; vasomotor; secretomotor; gastrointestinal; bladder; pupillomotor) with a total score ranging from 0 (normal) to 100 (worst condition). Additionally, patients were asked to fill out a modified visual analog scale (VAS) applied to 13 predefined major symptoms related to autonomic impairment (impaired quality of life, upright dizziness, inter-scapular pain during exercise, reduced sweating, reduced lacrimation, reduced salivation, urinary retention, urinary incontinence, reduced sexual desire, reduced sexual activity, dysphagia, constipation, visual acuity), where 0 corresponds to absence of symptoms and 10 to maximum intensity. One-way ANOVA for repeated measures followed by Dunnett's post hoc test were used to compare symptoms at baseline, at 1 and 2 years.

Results: Out of the 42 patients enrolled ( $36 \mathrm{~F}$; age $33.9 \pm 12.5 \mathrm{yrs}$ ), 1-year follow-up was achieved for $25(22 \mathrm{~F} ; 37.3 \pm 12.0$ years $)$ and 2-year follow-up was obtained for $12(10 \mathrm{~F}$; age $42.0 \pm 13.4$ years $)$. At baseline, the reported overall autonomic symptoms burden was high (overall COMPASS $31=49.9 / 100$ ). Main complaints were related to orthostatic intolerance according to both the COMPASS 31 (score 27.5/40) and VAS (score 5.82/10). Fourteen patients resulted inactive because of symptoms. Compared to baseline, at 1-year follow-up the COMPASS 31 detected a statistically significant improvement in pupillomotor function $(2.85 \pm 1.16$ vs. $2.44 \pm 1.01$; $\mathrm{p}<0.05$, respectively) and overall score $(50.64 \pm 14.13$ vs. $45.47 \pm 14.54, \mathrm{p}<0.05$; respectively). These findings were confirmed at 2 years along with a significant reduction in VAS quality of life impairment compared to baseline $(6.84 \pm 1.92$ vs. $5.44 \pm 2.49$; $\mathrm{p}<0.05$, respectively). However, these improvements were not associated with a change in the patients' occupational status.

Conclusion: Awareness of POTS diagnosis and frequent patients monitoring can help improve patients' condition.

Funding: The study was supported by an Italian Ministry of Health Grant \# RF-2013-02355242.

\section{Virtual Poster \#83}

POTS patients with excessive orthostatic tachycardia experience more benefits from body compression than patients with less orthostatic tachycardia

K.M. Bourne, J. Hall, R.S. Sheldon, D.V. Exner, J. Tyberg, S.R. Raj University of Calgary, Calgary, Alberta, Canada

Background: We have previously shown that compression garments can lower heart rate (HR) and improve symptoms in postural tachycardia syndrome (POTS), but the effects on patients with different orthostatic HR have not been investigated.

Objective: We hypothesized that participants with more significant tachycardia on head up tilt (HUT) would experience more benefits from compression than participants with lower HUT HR.

Methods: Participants completed $2 \times 10$ min HUTs in a randomized cross-over fashion, during one study day. A no compression control (NONE) and an abdomen/lower extremity compression intervention (FULL) were used. ECG and beat-to-beat blood pressure were recorded. Participants $(n=30,93 \%$ female) were assigned to a group based on their HUT heart rate (HR) increase with NONE: Group 1 ( $<30$ BPM), Group 2 (30-50 BPM) and Group 3 (>50 BPM). Group responses were compared using ANOVA tests. Data are presented as mean \pm standard error mean (SEM).

Results: The mean age of participants was $36 \pm 5$ years in Group 1 $(\mathrm{n}=8), 33 \pm 3$ years in Group $2(\mathrm{n}=14)$, and $27 \pm 3$ years in Group $3(\mathrm{n}=8 ; \mathrm{p}=0.2)$. Supine HR was not different between the 3 HR groups (Group 1: $72 \pm 5$ BPM, Group 2: $69 \pm 2$ BPM, Group $3: 72 \pm 3$ BPM, $p=0.8$ ). HR on HUT-NONE was $89 \pm 4$ BPM in Group 1, $107 \pm 2$ BPM in Group 2, and $134 \pm 3$ BPM in Group 3 $(\mathrm{p}<0.001)$. With HUT-FULL, improvement in HR (FULL-NONE) was greater in Group 3 ( $-28 \pm 3$ BPM), when compared to Group 2 $(-16 \pm 2$ BPM), and Group $1(-8 \pm 2$ BPM; $p<0.001)$. There was a non-significant trend with VOSS symptom scores on HUTNONE being higher in Group $3(35 \pm 7)$, when compared to Group 2 $(24 \pm 4)$ and Group $1(22 \pm 6 ; p=0.3)$. With HUT-FULL, improvement in VOSS symptom scores was not significantly different: Group $3(-18 \pm 5)$, compared to Group $2(-14 \pm 4)$, and Group $1(-10 \pm 3 ; \mathrm{p}=0.5)$.

Conclusion: Participants with orthostatic tachycardia $>50$ BPM experienced a significantly greater HR reduction with the addition of body compression on HUT than participants $<50$ BPM. Clinically, compression garments may be of greater benefit to patients with high orthostatic HR increases, than patients with moderate or low orthostatic HR increases.

\section{Virtual Poster \#84}

Phenylephrine alters phase synchronization between cerebral blood flow and blood pressure during orthostasis: effect on Nback performance in chronic fatigue syndrome/postural tachycardia syndrome

M.S. Medow ${ }^{1,2}$, C. Terilli ${ }^{1}$, J.M. Stewart ${ }^{1,2}$

Departments of ${ }^{1}$ Pediatrics and ${ }^{2}$ Physiology, New York Medical College, Valhalla, NY, USA

Chronic fatigue syndrome (CFS) with orthostatic intolerance is characterized by neuro-cognitive deficits and impaired working memory, concentration, and information processing. To evaluate this, we performed $\mathrm{N}$-back neurocognition testing and calculated the phase synchronization index $(\mathrm{PhSI})$ between $\mathrm{AP}$ and cerebral blood flow velocity $\left(\mathrm{CBF}_{\mathrm{V}}\right)$ as a nonlinear, nonstationary, time-dependent measurement of cerebral autoregulation in 11 control subjects (mean age $=24.1$ years) and 15 CFS patients (mean age $=21.8$ years). All patients were diagnosed with CFS but additionally met criteria for postural tachycardia syndrome (POTS). A $60^{\circ}$ head-up tilt (HUT) caused a significant increase in heart rate $(109.4 \pm 3.9$ vs. $77.2 \pm 1.6$ beats/min, $\mathrm{P}<0.05)$ and respiratory rate $(20.9 \pm 1.7$ vs. $14.2 \pm 1.2$ breaths/min, $\mathrm{P}<0.05)$ and decrease in end-tidal $\mathrm{CO}_{2} \quad\left(\mathrm{ETCO}_{2}\right.$; $33.9 \pm 1.1$ vs. $42.8 \pm 1.2$ Torr, $\mathrm{P}<0.05)$ in CFS vs. control. In CFS, HUT significantly decreased $(\mathrm{CBFv})$ compared to control $(-22.5 \%$ vs. $-8.7 \%, \mathrm{p}<0.005)$. To minimize the orthostatic reduction of $\mathrm{CBF}_{\mathrm{V}}$, we randomly administered supplemental $\mathrm{CO}_{2}$, phenylephrine or acetazolamide, and measured cardiopulmonary responses, supine and during HUT and performed N-back neurocognition testing control subjects and CFS patients. In CFS patients, these maneuvers each minimized the orthostatic decrease in brain blood flow such that HUT following $\mathrm{CO}_{2}$, phenylephrine (PE) and acetazolamide (Acet) resulted in $\mathrm{CBF}_{\mathrm{V}}$ that was equal to or greater than HUT control ( $-8.7 \%$ vs. $4.9 \%,-8.1 \%$ and $+6.6 \%)$, respectively. Despite this, only phenylephrine was able to correct the orthostatic decrease in neurocognition by reverting the $\%$ correct $n=4 \mathrm{~N}$-back values during HUT in CFS similar to control $(\mathrm{CFS}=38.5 \pm 5.5$ vs. $\mathrm{CFS}+\mathrm{PE}=$ 
$65.6 \pm 5.7$ vs. Control $56.9 \pm 7.5$ ). HUT in CFS resulted in elevated $\mathrm{PhSI}$ values indicating decreased cerebral autoregulation. While $\mathrm{CO}_{2}$ and Acet had no effect on PhSI in CFS patients, PE caused a significant reduction in $\mathrm{PhSI}$ (measured during $\mathrm{n}=4 \mathrm{~N}$-back, $\mathrm{CFS}=0.80 \pm 0.03$ vs. $\mathrm{CFS}+\mathrm{PE}=0.69 \pm 0.04, \mathrm{p}<0.05)$ that approached control values $(0.56 \pm 0.04)$. Thus, PE improved neurocognitive function in CFS patients, perhaps related to improved neurovascular coupling, cerebral autoregulation and maintenance of cerebral blood flow.

Funding: This study was supported by a grant from the Chronic Fatigue and Immune Deficiency Syndrome Association of America (M.S. Medow) and National Heart, Lung and Blood Institute grants R01 HL-112736 and R01 HL-074873 (J.M. Stewart).

\section{Virtual Poster \#85}

\section{Associated comorbid conditions contribute to gastrointestinal symptoms in postural tachycardia syndrome}

L. Millsap ${ }^{1}$, J. Langford ${ }^{1}$, J. Hemp ${ }^{3}$, M.M. Cortez ${ }^{1}$, L.A. Pace ${ }^{2}$ ${ }^{1}$ Department of Neurology, University of Utah, Salt Lake City, UT, USA; ${ }^{2}$ Center for Genomic Medicine, University of Utah, Salt Lake City, UT, USA; ${ }^{3}$ Internal Medicine, University of Utah, Salt Lake City, UT, USA

Postural tachycardia syndrome (POTS) is a heterogenous disorder, currently diagnosed by inappropriate tachycardia upon orthostatic challenge. Beyond orthostatic symptoms patients with POTS frequently report a variety of gastrointestinal (GI) and allergic symptoms although the pathophysiology of this association remains poorly understood. In recent years, POTS has been associated with a variety of comorbid conditions including the Ehlers-Danlos syndromes (EDS), mast cell activation syndrome (MCAS), median arcuate ligament syndrome (MALS), autonomic neuropathies (AN) and idiopathic GI dysmotility. Few studies, however, have systematically and comprehensively evaluated GI motility and presence of comorbid conditions in POTS patients. This retrospective analysis aimed to describe these conditions within a cohort of POTS seen at our institution. We included 106 patients seen for both autonomic neurology and neurogastroenterology evaluations at the University of Utah, between 2015 and 2020 (IRB_00080761). 38 of these individuals met consensus criteria for POTS. A retrospective chart review was performed to determine the presence of pertinent comorbid conditions, including EDS, MCAS, MALS, AN, and GI dysmotility. Of the 38 with patients with POTS (33 female/5 male; ages 18-49, median 33.5): $26 / 38$ (68.4\%) had EDS (23 with hEDS), 33/38 (86.8\%) had MCAS (including 3 with suspected MCAS), 23/38 (60.5\%) had MALS (including 5 with suspected MALS), 11 had AN diagnosed based on QSART, QST, and/or skin biopsy testing. Gastrointestinal motility was determined using the wireless motility capsule (WMC) and revealed segmental transit dysmotility in $37 / 38$ patients $(97.4 \%)$ and abnormal global transit times in 24/38 (63.2\%): 20 had abnormal gastric transit times, 14 had abnormal small bowel transit times, and 12 had abnormal colonic transit times. In this cohort of POTS patients, $100 \%$ of patients had diagnostic evidence to support at least one of the following comorbid conditions: EDS, MCAS, MALS, or AN. Furthermore, 37/38 (97.4\%) demonstrated quantifiable objective abnormalities in GI motility. These data support: (1) future studies to explore clinical subtypes of POTS patients based on comorbid condition burden, (2) future studies to explore the pathophysiology that contributes to this pattern of comorbid conditions, and (3) a role for quantitative objective gastrointestinal motility testing in patients with POTS.
Funding: Dysautonomia International, Grant No. DI 10049068 and National Institutes of Health, Office of Research on Women's Health, Grant No. K12HD085852 (to LAP).

\section{Virtual Poster \#86}

When sinus tachycardia becomes too much: negative effects of excessive upright tachycardia on cardiac output in vasovagal syncope, postural tachycardia syndrome, and inappropriate sinus tachycardia

J.M. Stewart ${ }^{1}$, M.S. Medow ${ }^{1}$, P. Visintainer ${ }^{2}$, R. Sutton ${ }^{3}$

${ }^{1}$ Hypotension Center, Departments of Pediatrics and Physiology, New York Medical College, Valhalla, NY, USA; ${ }^{2}$ Baystate Medical Center, Springfield, MA, USA; ${ }^{3}$ Imperial College National Heart \& Lung Institute, London, England

Background: Upright posture reduces venous return, stroke volume and cardiac output (CO) while causing reflex sinus rate (HR) increase. Yet, in inappropriate sinus tachycardia (IST), postural tachycardia syndrome (POTS), and vasovagal syncope (VVS) symptomatic excessive HR occurs. We hypothesized $\mathrm{CO}$ reaches maximum as function of HR in all.

Methods and Results: We recruited 12 healthy controls, 9 IST, 30 VVS and 30 POTS patients (13-23 years) selected randomly by disorder not by HR, each fulfilled appropriate diagnostic criteria. Subjects were instrumented for electrocardiography, beat-to-beat blood pressure, respiratory rate, CO-Modelflow algorithm, and central blood volume (CBV) from impedance cardiography; 10 min data was collected supine; subjects were tilted head-up for $\leq 10 \mathrm{~min}$. We computed phase differences, $\Delta \Phi$, between fluctuations of HR $(\Delta \mathrm{HR})$ and $\mathrm{CO}(\Delta \mathrm{CO})$, tabulating data when phases were synchronized, determined by a squared nonlinear phase synchronization index $(\mathrm{PhSI})>0.5$, describing extent/validity of $\mathrm{CO} / \mathrm{HR}$ coupling. We graphed results supine, 1 min-post-tilt-up, mid-tilt, and pre-tilt-down using polar coordinates (HR-radius, $\Delta \Phi$-angle) plotting $\cos (\Delta \Phi)$ vs. HR to determine if transition HR exists at which in-phase shifts to anti-phase above which $\mathrm{CO}$ decreases when HR further increases. At baseline HR, diastolic and mean arterial pressure in IST and POTS were higher vs. controls. Upright HR increased most in POTS then IST and VVS, with diverse changes in CO, SVR, and CBV. Each patient grouping was separately and collectively analyzed for HR change showing transition from in-phase to anti-phase $(\Delta \Phi)$ as HR increased: $\mathrm{HR}_{\text {transition }}=115 \pm 6$ (IST), $123 \pm 8$ (POTS), $124 \pm 7$ (VVS), $\mathrm{p}=\mathrm{ns}$. Controls never reached transitional HR.

Conclusions: Excessive HR independently and equivalently reduces upright CO, in IST, POTS and VVS.

Funding: Funding for this project was provided by grants R01 HL 112736 and R01 HL 134674 from the National Heart Lung and Blood Institute (NHLBI).

\section{Virtual Poster \#87}

The "extended" ANS, stress, and multi-system, multi-disciplinary disorders of regulation

\section{D.S. Goldstein}

Autonomic Medicine Section, CNP/DIR/NINDS/NIH, Bethesda, MD, USA

More than a century has gone by since Langley's definition of the autonomic nervous system (ANS) in terms of three components-the sympathetic nervous system, the parasympathetic nervous system, 
and the enteric nervous system. Now this conceptualization seems incomplete because of several developments in the interim. (1) Walter B. Cannon's demonstrations of adrenaline release into the bloodstream during emotional distress extended Langley's tripartite schema. Although Cannon viewed the sympathetic nervous and adrenomedullary hormonal systems as a monolithic entity (the "sympathico-adrenal" system) that would be recruited to maintain homeostasis in emergencies, actually the sympathetic noradrenergic system (SNS) is active tonically and plays key roles in all activities of daily life; and the sympathetic adrenergic system (SAS) is regulated differently from the SNS. The SAS probably was the first neuroendocrine system to be described, but over the past century several others have been discovered, including the vagal-pancreatic, corticotropin-releasing hormone-hypothalamic-pituitary-adrenocortical (HPA), vasopressin, and renin-angiotensin-aldosterone systems. (2) Stress emerged as a scientific concept. Hans Selye focused on the HPA axis and Walter B. Cannon on the sympathoadrenal system. Their constrained focuses prevented recognition of patterned alterations in effector activities. Selye's notion that stress is the nonspecific response of the body to any demand upon it failed experimental testing. Instead, I have proposed homeostatic definitions of stress and distress and "primitive specificity" of stress responses. Across stressors adrenaline responses are more closely tied to HPA than to SNS responses. (3) A central autonomic network (CAN) has been described. The CAN consists of complexly interacting neuronal clusters at all levels of the neuraxis, and Chrousos and Gold's "stress" system is embedded within the CAN. (4) There is expanding information about interactions between the ANS and the immune system. Autonomic neuroimmunology is a rapidly developing field that includes interplays between vagal-cholinergic-noradrenergic and inflammasome-cytokine systems. The extended ANS may help understand mechanisms and inform clinical management of multisystem, multi-disciplinary disorders of regulation. This presentation also conveys hypotheses about how activation of the extended ANS might contribute to the high morbidity and mortality associated with COVID-19 infection in the elderly and people with a variety of underlying chronic disorders.

Funding: Division of Intramural Research, NINDS, NIH.

\section{Virtual Poster \#88}

\section{Prevalence and patterns of abnormal QSWEAT volumes: a retrospective review}

T. Prieto, D.I. Sinn, S. Muppidi, M.G. Miglis, R. Shaik, S. Jaradeh Department of Neurology and Neurosciences, Stanford University, Stanford, CA, USA

Background: An interest in better understanding the prevalence, patterns, and sex-related differences of abnormal QSWEAT ${ }^{\mathrm{TM}}$ values led to this review.

Methods: We reviewed all Quantitative Sudomotor Axon Reflex Tests conducted using the QSWEAT ${ }^{\mathrm{TM}}$ (WR Medical Electronics, Maplewood, MN) in the Autonomic Disorders Testing Laboratory at Stanford from 1/1/16 to 3/31/20. All 10-min volume measurements were made on the standard sites (forearm (FA), proximal leg (PL), distal leg (DL), foot (FT)) with all lower limb sites unilateral. Volumes were classified as normal (NL), low normal (LN), and abnormal (AB) using normative values [Sletten et al., 2014]: NL: $>10 \%$ norm, $\mathrm{LN}: \leq 10 \%$ norm and $>5 \%$ norm, $\mathrm{AB} \leq 5 \%$ norm. Frequency of occurrence was calculated for each site and patterns in the lower limb for all subjects, females (F), and males (M).

Results: From the 2339 testing sessions in this time period, 2272 analyses were found that met the criteria $(1491 \mathrm{~F}, 781 \mathrm{M}$,
3-93 years). We narrowed the age range to match the normative values (20-69 years) and included the remaining 1534 analyses (1102 (71.8\%) F, $44.4 \pm 14.3$ years; $432(28.2 \%) \mathrm{M}, 49.0 \pm 15.5$ years). Of all sites, $71 \%$ were NL and $18 \% \mathrm{AB}$, with $44 \% \mathrm{NL}$ at all 4 sites and $3 \% \mathrm{AB}$ at all 4 sites. Females were more likely to have all sites NL (47\% F, 39\% M). Females were more likely to have AB FA (10\% $\mathrm{F}, 7 \% \mathrm{M})$. In the lower limb, all sites were equally likely $\mathrm{AB}$ in females, but in males, the DL was more likely $\mathrm{AB}$. The most common patterns in the lower limb (PL-DL-FT) were NL-NL-NL $46 \%$ and $\mathrm{AB}-\mathrm{AB}-\mathrm{AB} 8 \%$, followed by a length dependent pattern NL-NL-AB $4 \%$. NL-AB-NL was $5 \% \mathrm{M}$ and $1 \% \mathrm{~F}$.

Conclusions: We identified several patterns that may be clinically relevant. The normative data for the 20-69 age range are instrumental, but $32.5 \%$ of all subjects tested in this lab were outside this range, hence the need for normative values over a wider range. We did not include the referral diagnosis or review individual waveforms for this abstract but will when investigating some of these patterns further.

\section{Virtual Poster \#89}

Chronic use of baclofen and gabapentin in non-ambulatory, motor-incomplete persons with spinal cord injury modulates the change in systolic blood pressure to the seated upright posture

M.F. LaFountaine ${ }^{1,2,3}$, J.P. Weir ${ }^{4}$, C.G. Katzelnick ${ }^{1}$, M.T. Maher ${ }^{1}$, J.M. Wecht ${ }^{1,5}$

${ }^{1}$ VA RR\&D National Center for the Medical Consequences of SCI, James J. Peters VA Medical Center, Bronx, NY, USA; ${ }^{2}$ Department of Physical Therapy, School of Health and Medical Sciences, Seton Hall University, South Orange, NJ, USA; ${ }^{3}$ Departments of Medical Sciences and Neurology, Hackensack Meridian School of Medicine at Seton Hall University, Nutley, NJ, USA; ${ }^{4}$ Department of Health, Sport, and Exercise Sciences, University of Kansas, Lawrence, KS, USA; ${ }^{5}$ Department of Rehabilitation Medicine, Icahn School of Medicine at Mt. Sinai, New York, NY, USA

After a traumatic spinal cord injury (SCI) the cross-sectional extent to which the spinal cord is compromised may lead to a partial or complete loss of sensory, motor or sympathetic nervous system function below the level of injury. The clinical presentation arising from paralysis, autonomic dysfunction, and acclimation post-SCI may be characterized by limited medical consequences or represent a silent or significant symptomology requiring pharmaceutical intervention(s). The extent to which varying degrees of spared neurological function and pharmaceutical use impacts residual function is in the process of being defined. Ninety-three persons with chronic, non-ambulatory, motor-incomplete SCI [age (years): $40 \pm 11$ ] were evaluated during 5-min of supine, followed by 4-min of seated observation, while continuous 3-lead electrocardiogram and beat-to-beat SBP was recorded. A current medication history was obtained and categorized by the mechanism(s) of action. Fast-Fourier transform algorithms were used to calculate the high (HF), low (LF), and very low frequency (vLF) components of the SBP and heart rate (HR) signals, each parameter was $\log _{10}$ transformed. A change score between supine and seated positioning was calculated for each variable (delta_SBP, HF, LF and vLF, respectively). Stepwise regression analyses were performed to identify independent variables (e.g., demographic, autonomic outcomes and medications) with significant contributions to the observed delta_SBP from the supine to seated position. Significant predictors for the delta_SBP with seated upright posture were age, supine SBP, level of SCI (high paraplegia, low paraplegia, tetraplegia), delta_vLFSBP, baclofen, and gabapentin. This inclusive 
model accounted for a significant amount of the observed variance in the delta_SBP $\left(\mathrm{r}^{2} 0.919 ; \mathrm{p}<0.0001\right)$. Baclofen, being a $\mathrm{GABA}_{\mathrm{B}}$ agonist, activates central $\mathrm{GABA}_{\mathrm{B}}$ receptors to increase the inhibitory CNS actions of GABA. Gabapentin binds to specific voltage-gated calcium channels, which control many functions including skeletal muscle contractions. This latter consideration may help explain why the vLFSBP, which is described as being mediated by L-type calcium-dependent mechanisms and circulating catecholamines and angiotensin II, was identified as a robust contributor to the change in SBP with the seated positioning. Routine surveillance for the potential adverse consequences of blood pressure instability should be performed in persons with SCI who are prescribed baclofen and/or gabapentin.

Funding: Veteran Affairs Rehabilitation Research \& Development National Center for the Medical Consequences of Spinal Cord Injury \#B2020-C.

\section{Virtual Poster \#90}

\section{A cross sectional survey of autonomic nervous system dysfunction} in patients with mitochondrial disease

E.C. Shadiack III ${ }^{1}$, J.R. Boris $^{2}$, L. MacMullen ${ }^{1}$, E.M. McCormick ${ }^{1}$, I. George-Sankoh ${ }^{1}$, M.J. Falk ${ }^{1,3}$

${ }^{1}$ Mitochondrial Medicine Frontier Program, Division of Human Genetics, Department of Pediatrics, Children's Hospital of Philadelphia, Philadelphia, PA, USA; ${ }^{2}$ Pediatric Cardiologist, Philadelphia, PA, USA; ${ }^{3}$ Department of Pediatrics, University of Pennsylvania Perelman School of Medicine, Philadelphia, PA, USA

Introduction: Individuals with primary mitochondrial disease (PMD) may present with myriad signs and symptoms of affected multiple organ systems, including autonomic nervous system dysfunction (ANSd). Similarly, individuals with ANSd can present with a disparate array of widespread functional problems affecting cardiac, vascular, musculoskeletal, neurologic, endocrine, and gastrointestinal systems. Overlap between ANSd and mitochondrial dysfunction seems likely, but poorly defined. This study attempts to quantify the extent of ANSd symptoms in cohorts of patients with confirmed and suspected PMD.

Methods: Subjects were solicited from the Children's Hospital of Philadelphia Mitochondrial Medicine Frontier Program's Metabolic Consequences of Primary Mitochondrial Disease (CHOP) cohort (CHOP IRB 16-013320), with a validation cohort solicited from the Mitochondrial Disease Community Registry (MDCR) (CHOP IRB16013329). The Dysautonomia Symptom Survey (DySSy) was developed to evaluate ANSd symptoms. Individuals were given the Composite Autonomic Symptom Score 31 (COMPASS-31) (0-100; higher scores representing higher symptom burden) as comparator both for the DySSy survey subjects and for ANSd in previous ANS patient case series.

Results: Eighty-eight subjects were included in the initial $\mathrm{CHOP}$ PMD cohort analysis (46 adult and 42 pediatric; 47 females and 41 males). Nineteen adults (41.3\%) and 22 children (52.4\%) had molecularly confirmed PMD. Upon DySSY analyses, subjects with suspected PMD reported more bloating $(\mathrm{p}=0.02)$, tachycardia $(p=0.003)$, heat intolerance $(p=0.001)$, and skin color changes $(\mathrm{P}=0.007)$, whereas definite PMD subjects reported more cold intolerance $(\mathrm{p}=0.009)$. Total COMPASS-31 scores in definite PMD (18.1) were lower versus suspected PMD (30.2, p < 0.001). Definite PMD scores were higher in adults ( $\geq 18$ years, 22.5) versus children $(14.3, p=0.05)$. Scores were higher in definite PMD (18.1) versus historically-reported healthy controls $(8.9, \mathrm{p}=0.005)$, but were lower compared to reported cohorts with small fiber polyneuropathy (30.2, $\mathrm{p}=0.004)$, diabetic neuropathy $(28.9, \mathrm{p}=0.01)$, scleroderma $(24.9$, $\mathrm{p}=0.02)$, and fibromyalgia $(27.5, \mathrm{p}=0.02)$.

Conclusion: This is the first systematic study to quantify ANSd in subjects with definite and suspected PMD. While ANSd is present in the PMD population, with greater prevalence in adults than children, they have less symptom burden versus other populations with ANSd. Interestingly, preliminary analyses suggest ANSd significantly differs between subjects with definite PMD and suspected PMD. 